\title{
Minimax-Robust Forecasting of Sequences with Periodically Stationary Long Memory Multiple Seasonal Increments
}

\author{
Maksym Luz, Mikhail Moklyachuk* \\ Department of Probability Theory, Statistics and Actuarial Mathematics, Taras Shevchenko National University of Kyiv, Ukraine
}

\begin{abstract}
We introduce stochastic sequences $\zeta(k)$ with periodically stationary generalized multiple increments of fractional order which combines cyclostationary, multi-seasonal, integrated and fractionally integrated patterns. We solve the problem of optimal estimation of linear functionals constructed from unobserved values of stochastic sequences $\zeta(k)$ based on their observations at points $k<0$. For sequences with known spectral densities, we obtain formulas for calculating values of the mean square errors and the spectral characteristics of the optimal estimates of functionals. Formulas that determine the least favorable spectral densities and minimax (robust) spectral characteristics of the optimal linear estimates of functionals are proposed in the case where spectral densities of sequences are not exactly known while some sets of admissible spectral densities are given.
\end{abstract}

Keywords Periodically stationary sequence, SARFIMA, fractional integration, optimal linear estimate, mean square error, least favourable spectral density, minimax spectral characteristic

AMS 2010 subject classifications. Primary: 60G10, 60G25, 60G35

DOI: $10.19139 /$ soic-2310-5070-998

\section{Introduction}

A variety of non-stationary and long memory time series models are introduced and investigated by researchers in the past decade (see, for example, papers by Dudek and Hurd [9], Johansen and Nielsen [24], Reisen et al.[44]). Such models are used when analyzing data which arise in different field of economics, finance, climatology, air pollution, signal processing.

Since the book by Box and Jenkins (1970), autoregressive moving average (ARMA) models integrated of order $d$ are standard models used for time series analysis. These models are described by the equation

$$
\psi(B)(1-B)^{d} x_{t}=\theta(B) \varepsilon_{t},
$$

where $\varepsilon_{t}, t \in \mathbb{Z}$, is a sequence of zero mean i.i.d. random variables, $\psi(z), \theta(z)$ are polynomials of $p$ and $q$ degrees respectively with roots outside the unit circle. This integrated ARIMA model is generalized by adding a seasonal component. A new model is described by the equation (see new edition of the book by Box and Jenkins [6] for detailes)

$$
\Psi\left(B^{s}\right)\left(1-B^{s}\right)^{D} x_{t}=\Theta\left(B^{s}\right) \varepsilon_{t},
$$

where $\Psi(z)$ and $\Theta(z)$ are polynomials of degrees of $P$ and $Q$ respectively which have roots outside the unit circle.

When an ARIMA sequence determined by equation (1) is inserted in relation (2) instead of $\varepsilon_{t}$ we have general multiplicative model

$$
\Psi\left(B^{s}\right) \psi(B)(1-B)^{d}\left(1-B^{s}\right)^{D} x_{t}=\Theta\left(B^{s}\right) \theta(B) \varepsilon_{t}
$$

\footnotetext{
*Correspondence to: Mikhail Moklyachuk (Email: moklyachuk@gmail.com). Department of Probability Theory, Statistics and Actuarial Mathematics, Taras Shevchenko National University of Kyiv, Volodymyrska 64 Str., Kyiv 01601, Ukraine.
}

ISSN 2310-5070 (online) ISSN 2311-004X (print)

Copyright (C) 2020 International Academic Press 
with parameters $(p, d, q) \times(P, D, Q)_{s}, d, D \in \mathbb{N}^{*}$, called SARIMA $(p, d, q) \times(P, D, Q)_{s}$ model.

A good performance is shown by models which include fractional integration, that is when parameters $d$ and $D$ are fractional. When $|d+D|<1 / 2$ and $|D|<1 / 2$, a process described by equation (3) is stationary and invertible. We refer to the paper by Porter-Hudak [43] who studied seasonal ARFIMA models and applied them to the monetary aggregates used by U.S. Federal Reserve.

Closely related to fractionally integrated ARMA and GARMA processes described by equation

$$
\left(1-2 u B+B^{2}\right)^{d} x_{t}=\varepsilon_{t}, \quad|u| \leq 1 .
$$

is SARFIMA process. These processes were introduced and studied by Granger and Joyeux [15], Hosking [21], Andel [1], Gray et al. [17] in order to model long-memory stationary time series. Fractionally integrated models are a powerful tool for studying a variety of real world processes. For the resent works dedicated to statistical inference for seasonal long-memory sequences, we refer to Arteche and Robinson [2], who applied the log-periodogram and Gaussian or Whittle methods of memory parameters estimation for seasonal/cyclical asymmetric long memory processes with application to UK inflation data, and also Tsai, Rachinger and Lin [48], who developed methods of estimation of parameters in case of measurement errors. Baillie, Kongcharoen and Kapetanios [4] compared MLE and semiparametric estimation procedures for prediction problems based on ARFIMA models. Based on simulation study, they indicate better performance of MLE predictor than the one based on two-step local Whittle estimation. Hassler and Pohle [20] (see also Hassler [19]) assess a predictive performance of various methods of forecasting of inflation and return volatility time series and show strong evidences for models with a fractional integration component.

Another type of non-stationarity is described by stochastic processes with time-dependent spectrum. A wide class of processes with time-dependent spectrum is formed by periodically correlated, or cyclostationary, processes introduced by Gladyshev [13]. These processes are widely used in signal processing and communications (see Napolitano [39] for a review of recent works on cyclostationarity and its applications). Periodic time series may be considered as an extension of a SARIMA model (see Lund [30] for a test assessing if a PARMA model is preferable to a SARMA one) and are suitable for forecasting stream flows with quarterly, monthly or weekly cycles (see Osborn [40]).

Baek, Davis and Pipiras [3] introduced a periodic dynamic factor model (PDFM) with periodic vector autoregressive (PVAR) factors, in contrast to seasonal VARIMA factors.

The models mentioned above are used in estimation of model's parameters and forecast issues. Meanwhile a direct application of the developed results to real data may lead to significant increasing of errors of estimates due to presence of outliers, measurement errors, incomplete information about the spectral, or model, structure etc. From this point of view, we see an increasing interest to robust methods of estimation that are reasonable in such cases. For example, Reisen, et al. [45] proposed a semiparametric robust estimator for fractional parameters in the SARFIMA model and illustrated its application to forecast of sulfur dioxide $\mathrm{SO}_{2}$ pollutant concentrations. Solci at al. [47] proposed robust estimates of periodic autoregressive (PAR) model.

Robust approaches are successfully applied to the problem of estimation of linear functionals from unobserved values of stochastic processes. The paper by Grenander [16] should be marked as the first one where the minimax extrapolation problem for stationary processes was formulated as a game of two players and solved. Hosoya [22], Kassam [25], Franke [10], Vastola and Poor [49], Moklyachuk [33, 34] studied minimax extrapolation (forecasting), interpolation (missing values estimation) and filtering (smoothing) problems for stationary sequences and processes. Recent results of minimax extrapolation problems for stationary vector-valued processes and periodically correlated processes belong to Moklyachuk and Masyutka [35, 36] and Moklyachuk and Golichenko (Dubovetska) [7] respectively. Processes with stationary increments are investigated by Moklyachuk and Luz $[31,32]$. We also mention works by Moklyachuk and Sidei [37, 38], who derive minimax estimates of stationary processes from observations with missed values. Moklyachuk and Kozak [29] studied interpolation problem for stochastic sequences with periodically stationary increments.

In this article, we present results of investigation of stochastic sequences with periodically stationary long memory multiple seasonal increments motivated by articles by Dudek [8], Gould et al. [14] and Reisen et al. [44], who considered models with multiple seasonal patterns for inference and forecasting, and Hurd and Piparas [23], who introduced two models of periodic autoregressive time series with multiple periodic coefficients. 
In Section 2, we give definition of generalized multiple (GM) increment sequence $\chi_{\bar{\mu}, \bar{s}}^{(d)}(\vec{\xi}(m))$ and introduce stochastic sequences $\zeta(m)$ with periodically stationary (periodically correlated, cyclostationary) GM increments. Such kind of non-stationary stochastic sequence combines periodic structure of the covariation function of the sequences as well as multiple seasonal factors, including the integrating one. The section also contains a short review of the spectral theory of vector-valued GM increment sequences. Section 3 deals with the classical estimation problem for linear functionals $A \zeta$ and $A_{N} \zeta$ which are constructed from unobserved values of the sequence $\zeta(m)$ when the spectral structure of the sequence $\zeta(m)$ is known. Estimates are obtained by representing the sequence $\zeta(m)$ as a vector sequence $\vec{\xi}(m)$ with stationary GM increments and applying the Hilbert space projection technique. An approach to forecasting in the presence of non-stationary fractional integration is discussed in Section 4. Section 5 contains examples of forecasting of particular models of time series. In Section 6, we derive the minimax (robust) estimates in cases, where spectral densities of sequences are not exactly known while some sets of admissible spectral densities are specified which are generalizations of the corresponding sets of admissible spectral densities described in a survey article by Kassam and Poor [26] for stationary stochastic processes.

\section{Stochastic sequences with periodically stationary generalized multiple increments}

\subsection{Definition and spectral representation of a periodically stationary GM increment}

In this section, we present definition, justification and a brief review of the spectral theory of stochastic sequences with periodically stationary multiple seasonal increments. This type of stochastic sequences will allow us to deal with a wide range of non-stationarity in time series analysis.

Consider a stochastic sequence $\{\eta(m), m \in \mathbb{Z}\}$. By $B_{\mu}$ denote a backward shift operator with the step $\mu \in \mathbb{Z}$, such that $B_{\mu} \eta(m)=\eta(m-\mu) ; B:=B_{1}$. Recall the following definition [32, 42, 52].

Definition 2.1

For a given stochastic sequence $\{\eta(m), m \in \mathbb{Z}\}$, the sequence

$$
\eta^{(n)}(m, \mu)=\left(1-B_{\mu}\right)^{n} \eta(m)=\sum_{l=0}^{n}(-1)^{l}\left(\begin{array}{l}
n \\
l
\end{array}\right) \eta(m-l \mu),
$$

where $\left(\begin{array}{l}n \\ l\end{array}\right)=\frac{n !}{l !(n-l) !}$, is called stochastic $n$th increment sequence with a step $\mu \in \mathbb{Z}$.

Note that in Definition 2.1, the step parameter $\mu$ is not fixed and varies over the set $\mathbb{Z}$. The introduced increment sequence (5) is applicable for describing the integrated stochastic sequence (1). The varying step $\mu$ provides a flexibility of the integrated sequences. For instance, let a sequence $x_{m}$ satisfy the equation

$$
x_{m}=x_{m-1}+\varepsilon_{m}+a \varepsilon_{m-1} .
$$

Then the $\mu$-step increment

$$
x_{m}-x_{m-\mu}=\sum_{k=0}^{\mu-1}\left(x_{m-k}-x_{m-k-1}\right)
$$

is stationary as a sum of stationary 1-step increments. To deal with seasonal time series (2) we need to extend definition of stochastic increment sequence as follows.

Definition 2.2

For a given stochastic sequence $\{\eta(m), m \in \mathbb{Z}\}$, the sequence

$$
\eta_{s}^{(n)}(m, \mu)=\left(1-B_{\mu}^{s}\right)^{n} \eta(m)=\sum_{l=0}^{n}(-1)^{l}\left(\begin{array}{c}
n \\
l
\end{array}\right) \eta(m-l \mu s)
$$

is called stochastic seasonal increment sequence with a fixed seasonal parameter $s \in \mathbb{N}^{*}=\mathbb{N} \backslash\{0\}$ and a varying step $\mu \in \mathbb{Z}$. 
Remark 2.1

For $s=1$, under the seasonal increment $\eta_{1}^{(n)}(m, \mu)$ we understand the increment $\eta^{(n)}(m, \mu)$ from Definition 2.1.

We mention the following properties of the seasonal increment sequence $\eta_{s}^{(n)}(m, \mu)$, which will be used for proving Theorem 2.2:

$$
\begin{aligned}
\eta_{s}^{(n)}(m,-\mu) & =(-1)^{n} \eta_{s}^{(n)}(m+n \mu s, \mu), \\
\eta_{s}^{(n)}(m, \mu) & =\sum_{l=0}^{(\mu-1) n} A_{l} \eta^{(n)}(m-l s, 1), \quad \mu>0,
\end{aligned}
$$

where $\left\{A_{l}, l=0,1,2, \ldots,(\mu-1) n\right\}$ are coefficients from the representation

$$
\left(1+x+\ldots+x^{\mu-1}\right)^{n}=\sum_{l=0}^{(\mu-1) n} A_{l} x^{l} .
$$

General multiplicative model (3) [6] indicates the necessity of dealing with increments of different seasonal parameters. Moreover, for each season factor at each differencing order it is possible to make different steps by applying the operator $\left(1-B_{\mu_{1}}^{s}\right) \cdot \ldots \cdot\left(1-B_{\mu_{n}}^{s}\right)$ instead of $\left(1-B_{\mu}^{s}\right)^{n}$. Thus, the following generalization is reasonable.

Definition 2.3

For a given stochastic sequence $\{\eta(m), m \in \mathbb{Z}\}$, the sequence

$$
\begin{gathered}
\chi_{\bar{\mu}, \bar{s}}^{(d)}(\eta(m)):=\chi_{\bar{\mu}, \bar{s}}^{(d)}(B) \eta(m)=\left(1-B_{\mu_{1}}^{s_{1}}\right)^{d_{1}}\left(1-B_{\mu_{2}}^{s_{2}}\right)^{d_{2}} \cdot \ldots \cdot\left(1-B_{\mu_{r}}^{s_{r}}\right)^{d_{r}} \eta(m) \\
\quad=\sum_{l_{1}=0}^{d_{1}} \ldots \sum_{l_{r}=0}^{d_{r}}(-1)^{l_{1}+\ldots+l_{r}}\left(\begin{array}{c}
d_{1} \\
l_{1}
\end{array}\right) \cdot \ldots \cdot\left(\begin{array}{c}
d_{r} \\
l_{r}
\end{array}\right) \eta\left(m-\mu_{1} s_{1} l_{1}-\cdots-\mu_{r} s_{r} l_{r}\right)
\end{gathered}
$$

is called stochastic generalized multiple (GM) increment sequence of differentiation order $d:=d_{1}+d_{2}+\ldots+d_{r}$, $\bar{d}=\left(d_{1}, d_{2}, \ldots, d_{r}\right) \in\left(\mathbb{N}^{*}\right)^{r}$, with a fixed seasonal vector $\bar{s}=\left(s_{1}, s_{2}, \ldots, s_{r}\right) \in\left(\mathbb{N}^{*}\right)^{r}$ and a varying step $\bar{\mu}=$ $\left(\mu_{1}, \mu_{2}, \ldots, \mu_{r}\right) \in\left(\mathbb{N}^{*}\right)^{r}$ or $\in(\mathbb{Z} \backslash \mathbb{N})^{r}$.

Example 2.1

Seasonal autoregressive integrated moving average (SARIMA) model $\left\{x_{m}, m \in \mathbb{Z}\right\}$ with multiple period is defined by the difference equation

$$
\phi(B)(1-B)^{d} \prod_{i=1}^{r} \Phi_{i}\left(B^{s_{i}}\right)\left(1-B^{s_{i}}\right)^{d_{i}} x_{m}=\theta(B) \prod_{i=1}^{r} \Theta_{i}\left(B^{s_{i}}\right) \varepsilon_{m},
$$

where all roots of polynomials $\phi(z), \theta(z), \Phi_{i}(z), \Theta_{i}(z)$ lie outside the unit circle, $1<s_{1}<\ldots<s_{r}$. The sequence $y_{m}=(1-B)^{d} \prod_{i=1}^{r}\left(1-B^{s_{i}}\right)^{d_{i}} x_{m}$ is stationary in this case and we can define a GM increment sequence $\chi_{\bar{\mu}, \bar{s}}^{(d)}\left(x_{m}\right)$ such that $\chi_{\overline{1}, \bar{s}}^{(d)}\left(x_{m}\right)=y_{m}, m \in \mathbb{Z}$.

Let $\gamma$ denotes a triple $(\bar{\mu}, \bar{s}, \bar{d})$. For $i=1,2, \ldots, r, j \in \mathbb{Z}$ define coefficients $M_{i}^{j}:=\left[\frac{j}{\mu_{i} s_{i}}\right]$ and $I_{i}^{j}:=\mathbb{I}\{j$ $\left.\bmod \mu_{i} s_{i}=0\right\}$, where $\mathbb{I}\{\cdot\}$ is the indicator function, and notations $n_{i}:=\mu_{i} s_{i} d_{i},\langle\bar{s}, \bar{\mu}, \bar{d}\rangle_{k}:=\sum_{i=1}^{k} \mu_{i} s_{i} d_{i}=$ $\sum_{i=1}^{k} n_{i}, n(\gamma):=\langle\bar{s}, \bar{\mu}, \bar{d}\rangle_{r}$. Denote the maximun of two numbers as $x \vee y$ and the minimum as $x \wedge y$.

\section{Lemma 2.1}

The multiplicative increment operator $\chi_{\bar{\mu}, \bar{s}}^{(d)}(B)$ admits a representation

$$
\chi_{\bar{\mu}, \bar{s}}^{(d)}(B)=\prod_{i=1}^{r}\left(1-B_{\mu_{i}}^{s_{i}}\right)^{d_{i}}=\sum_{k=0}^{n(\gamma)} e_{\gamma}(k) B^{k}
$$




$$
e_{\gamma}(k)=\sum_{k_{r-1}=0 \vee k_{r}-n_{r}}^{\langle\bar{\mu}, \bar{\mu}, \bar{d}\rangle_{r-1} \wedge k_{r}} \sum_{k_{r-2}=0 \vee k_{r-1}-n_{r-1}}^{\langle\bar{s}, \bar{\mu}, \bar{d}\rangle_{r-2} \wedge k_{r-1}} \cdots \sum_{k_{1}=0 \vee k_{2}-n_{2}}^{\langle\bar{s}, \bar{\mu}, \bar{d}\rangle_{1} \wedge k_{2}}\left((-1)^{\sum_{i=1}^{r} M_{i}^{k_{i}-k_{i-1}}} \prod_{i=1}^{r} I_{i}^{k_{i}-k_{i-1}} \prod_{i=1}^{r}\left(\begin{array}{c}
d_{i} \\
M_{i}^{k_{i}-k_{i-1}}
\end{array}\right)\right),
$$

where $k_{0}:=0, k_{r}:=k$.

Proof

See Appendix.

\section{Definition 2.4}

A stochastic GM increment sequence $\chi_{\bar{\mu}, \bar{s}}^{(d)}(\eta(m))$ generated by a stochastic sequence $\{\eta(m), m \in \mathbb{Z}\}$ is wide sense stationary if the mathematical expectations

$$
\begin{aligned}
\mathrm{E} \chi_{\bar{\mu}, \bar{s}}^{(d)}\left(\eta\left(m_{0}\right)\right) & =c_{\bar{s}}^{(d)}(\bar{\mu}), \\
\mathrm{E} \chi_{\bar{\mu}_{1}, \bar{s}}^{(d)}\left(\eta\left(m_{0}+m\right)\right) \chi_{\bar{\mu}_{2}, \bar{s}}^{(d)}\left(\eta\left(m_{0}\right)\right) & =D_{\bar{s}}^{(d)}\left(m ; \bar{\mu}_{1}, \bar{\mu}_{2}\right)
\end{aligned}
$$

exist for all $m_{0}, m, \bar{\mu}, \bar{\mu}_{1}, \bar{\mu}_{2}$ and do not depend on $m_{0}$. The function $c_{\bar{s}}^{(d)}(\bar{\mu})$ is called mean value and the function $D_{\bar{s}}^{(d)}\left(m ; \bar{\mu}_{1}, \bar{\mu}_{2}\right)$ is called structural function of the stationary GM increment sequence.

The stochastic sequence $\{\eta(m), m \in \mathbb{Z}\}$ determining the stationary GM increment sequence $\chi_{\bar{\mu}, \bar{s}}^{(d)}(\eta(m))$ by (9) is called stochastic sequence with stationary GM increments (or GM increment sequence of order $d$ ).

The following theorem is a generalization of the corresponding theorem for stochastic increment sequence $\eta^{(d)}(m, \mu)[32,51]$.

\section{Theorem 2.1}

The mean value and the structural function of the stochastic stationary GM sequence $\chi_{\bar{\mu}, \bar{s}}^{(d)}(\eta(m))$ can be represented in the forms

$$
\begin{aligned}
c_{\bar{s}}^{(d)}(\bar{\mu}) & =c \prod_{i=1}^{r} \mu_{i}^{d_{i}} \\
D_{\bar{s}}^{(d)}\left(m ; \bar{\mu}_{1}, \bar{\mu}_{2}\right) & =\int_{-\pi}^{\pi} e^{i \lambda m} \chi_{\bar{\mu}_{1}}^{(d)}\left(e^{-i \lambda}\right) \chi_{\bar{\mu}_{2}}^{(d)}\left(e^{i \lambda}\right) \frac{1}{\left|\beta^{(d)}(i \lambda)\right|^{2}} d F(\lambda),
\end{aligned}
$$

where

$$
\chi_{\bar{\mu}}^{(d)}\left(e^{-i \lambda}\right)=\prod_{j=1}^{r}\left(1-e^{-i \lambda \mu_{j} s_{j}}\right)^{d_{j}}, \quad \beta^{(d)}(i \lambda)=\prod_{j=1}^{r} \prod_{k_{j}=-\left[s_{j} / 2\right]}^{\left[s_{j} / 2\right]}\left(i \lambda-2 \pi i k_{j} / s_{j}\right)^{d_{j}},
$$

$c$ is a constant, $F(\lambda)$ is a left-continuous nondecreasing bounded function. The constant $c$ and the function $F(\lambda)$ are determined uniquely by the GM increment sequence $\chi_{\bar{\mu}, \bar{s}}^{(d)}(\eta(m))$.

On the other hand, a function $c_{\bar{s}}^{(d)}(\mu)$ which has form (10) with a constant $c$ and a function $D_{\bar{s}}^{(d)}\left(m ; \bar{\mu}_{1}, \bar{\mu}_{2}\right)$ which has form (11) with a function $F(\lambda)$ satisfying the indicated conditions are the mean value and the structural function of a stationary GM increment sequence $\chi_{\bar{\mu}, \bar{s}}^{(d)}(\eta(m))$.

\section{Proof}

See Appendix.

Note that by the spectral function and the spectral density of a stochastic sequence with stationary GM increments, we will call the spectral function and the spectral density of the corresponding stationary GM increment sequence. Representation (11) and the Karhunen theorem [27, 11] imply the spectral representation of the stationary GM increment sequence $\chi_{\bar{\mu}, \bar{s}}^{(d)}(\eta(m))$ :

$$
\chi_{\bar{\mu}, \bar{s}}^{(d)}(\eta(m))=\int_{-\pi}^{\pi} e^{i m \lambda} \chi_{\bar{\mu}}^{(d)}\left(e^{-i \lambda}\right) \frac{1}{\beta^{(d)}(i \lambda)} d Z_{\eta^{(d)}}(\lambda),
$$


where $Z_{\eta^{(d)}}(\lambda)$ is a stochastic process with uncorrelated increments on $[-\pi, \pi)$ connected with the spectral function $F(\lambda)$ by the relation

$$
\mathrm{E}\left|Z_{\eta^{(d)}}\left(\lambda_{2}\right)-Z_{\eta^{(d)}}\left(\lambda_{1}\right)\right|^{2}=F\left(\lambda_{2}\right)-F\left(\lambda_{1}\right)<\infty, \quad-\pi \leq \lambda_{1}<\lambda_{2}<\pi .
$$

Finally, we are ready to give a definition of periodically stationary GM increment sequence.

\section{Definition 2.5}

A stochastic sequence $\{\zeta(m), m \in \mathbb{Z}\}$ is called stochastic sequence with periodically stationary (periodically correlated) GM increments with period $T$ if the mathematical expectations

$$
\begin{aligned}
\mathrm{E} \chi_{\bar{\mu}, T \bar{s}}^{(d)}(\zeta(m+T)) & =\mathrm{E} \chi_{\bar{\mu}, T \bar{s}}^{(d)}(\zeta(m))=c_{T \bar{s}}^{(d)}(m, \bar{\mu}), \\
\mathrm{E} \chi_{\bar{\mu}_{1}, T \bar{s}}^{(d)}(\zeta(m+T)) \chi_{\bar{\mu}_{2}, T \bar{s}}^{(d)}(\zeta(k+T)) & =D_{T \bar{s}}^{(d)}\left(m+T, k+T ; \bar{\mu}_{1}, \bar{\mu}_{2}\right)=D_{T \bar{s}}^{(d)}\left(m, k ; \bar{\mu}_{1}, \bar{\mu}_{2}\right)
\end{aligned}
$$

exist for every $m, k, \bar{\mu}_{1}, \bar{\mu}_{2}$ and $T>0$ is the least integer for which these equalities hold.

It follows from Definition 2.5 that the sequence

$$
\xi_{p}(m)=\zeta(m T+p-1), \quad p=1,2, \ldots, T ; \quad m \in \mathbb{Z}
$$

forms a vector-valued sequence $\vec{\xi}(m)=\left\{\xi_{p}(m)\right\}_{p=1,2, \ldots, T}, m \in \mathbb{Z}$ with stationary GM increments as follows:

$$
\begin{aligned}
\chi_{\bar{\mu}, \bar{s}}^{(d)}\left(\xi_{p}(m)\right) & =\sum_{l_{1}=0}^{d_{1}} \ldots \sum_{l_{r}=0}^{d_{r}}(-1)^{l_{1}+\ldots+l_{r}}\left(\begin{array}{c}
d_{1} \\
l_{1}
\end{array}\right) \cdot \ldots \cdot\left(\begin{array}{c}
d_{r} \\
l_{r}
\end{array}\right) \xi_{p}\left(m-\mu_{1} s_{1} l_{1}-\ldots-\mu_{r} s_{r} l_{r}\right) \\
& =\sum_{l_{1}=0}^{d_{1}} \ldots \sum_{l_{r}=0}^{d_{r}}(-1)^{l_{1}+\ldots+l_{r}}\left(\begin{array}{c}
d_{1} \\
l_{1}
\end{array}\right) \cdot \ldots \cdot\left(\begin{array}{c}
d_{r} \\
l_{r}
\end{array}\right) \zeta\left(\left(m-\mu_{1} s_{1} l_{1}-\ldots-\mu_{r} s_{r} l_{r}\right) T+p-1\right) \\
& =\chi_{\bar{\mu}, T \bar{s}}^{(d)}(\zeta(m T+p-1)), \quad p=1,2, \ldots, T
\end{aligned}
$$

where $\chi_{\bar{\mu}, \bar{s}}^{(d)}\left(\xi_{p}(m)\right)$ is the GM increment of the $p$-th component of the vector-valued sequence $\vec{\xi}(m)$. The following theorem describes a spectral representation of the sequence $\vec{\xi}(m)$.

Example 2.2

Define a periodic seasonal autoregressive integrated moving average model (PSARIMA) $\left\{X_{m}, m \in \mathbb{Z}\right\}$, with multiple seasonal patterns by relation

$$
\phi_{m}(B)\left(1-B^{T}\right)^{d} \prod_{i=1}^{r} \Phi_{i, m}(B)\left(1-B^{T s_{i}}\right)^{d_{i}} X_{m}=\theta_{m}(B) \prod_{i=1}^{r} \Theta_{i, m}(B) \varepsilon_{m},
$$

where all polynomials $\phi_{m}(z), \theta_{m}(z), \Phi_{i, m}(z), \Theta_{i, m}(z)$ are $T$-periodic by parameter $m$ functions, $1<s_{1}<\ldots<$ $s_{r}$. Define

$$
\begin{aligned}
\Phi_{m}(z) & :=\phi_{m}(z) \prod_{i=1}^{r} \Phi_{i, m}(z)=\sum_{k=0}^{q_{1}} \Phi_{m}(k) z^{k}, \\
\Theta_{m}(z) & :=\theta_{m}(z) \prod_{i=1}^{r} \Theta_{i, m}(z)=\sum_{k=0}^{q_{2}} \Theta_{m}(k) z^{k}
\end{aligned}
$$

and put $\Phi_{m}(k)=0$ for $k>q_{1}, \Theta_{m}(k)=0$ for $k>q_{2}$. Then the increment sequence

$$
Y_{m}=\left(1-B^{T}\right)^{d} \prod_{i=1}^{r}\left(1-B^{T s_{i}}\right)^{d_{i}} X_{m}
$$


is periodically stationary and allows a stationary vector representation

$$
\mathbf{Y}_{m}=(1-B)^{d} \prod_{i=1}^{r}\left(1-B^{s_{i}}\right)^{d_{i}} \mathbf{X}_{m}
$$

with

$$
\mathbf{Y}_{m}=\left(Y_{m T}, Y_{m T+1}, \ldots, Y_{m T+T-1}\right)^{\top}, \quad \mathbf{X}_{m}=\left(X_{m T}, X_{m T+1}, \ldots, X_{m T+T-1}\right)^{\top},
$$

$\varepsilon_{m}=\left(\varepsilon_{m T}, \varepsilon_{m T+1}, \ldots, \varepsilon_{m T+T-1}\right)^{\top}$. We can write the relation

$$
\boldsymbol{\Pi} \mathbf{Y}_{m}+\sum_{l=1}^{q_{1}^{*}} \boldsymbol{\Pi}_{l} \mathbf{Y}_{m-l}=\boldsymbol{\Xi} \boldsymbol{\varepsilon}_{m}+\sum_{l=1}^{q_{2}^{*}} \boldsymbol{\Xi}_{l} \boldsymbol{\varepsilon}_{m-l}
$$

where $\boldsymbol{\Pi}(k, j)=\Phi_{k}(k-j), \boldsymbol{\Xi}(k, j)=\Theta_{k}(k-j)$ for $k \geq j, \boldsymbol{\Pi}(k, j)=0, \boldsymbol{\Xi}(k, j)=0$ otherwise. $\boldsymbol{\Pi}_{l}(k, j)=$ $\Phi_{k}(l T+k-j), \boldsymbol{\Xi}_{l}(k, j)=\Theta_{k}(l T+k-j)$ [9], provided $\operatorname{det}\left(\boldsymbol{\Pi}+\sum_{l=1}^{q_{1}^{*}} \boldsymbol{\Pi}_{l} z^{l}\right) \neq 0$ for $|z| \leq 1$ [18]. A GM increment sequence is defined as

$$
\chi_{\bar{\mu}, \bar{s}}^{(d)}\left(\mathbf{X}_{m}\right)=\left(1-B^{\mu_{0}}\right)^{d} \prod_{i=1}^{r}\left(1-B^{s_{i} \mu_{i}}\right)^{d_{i}} \mathbf{X}_{m}, m \in \mathbb{Z} .
$$

Theorem 2.2

The structural function $D_{\bar{s}}^{(d)}\left(m ; \bar{\mu}_{1}, \bar{\mu}_{2}\right)$ of the vector-valued stochastic stationary GM increment sequence $\chi_{\bar{\mu}, \bar{s}}^{(d)}(\vec{\xi}(m))$ can be represented in the form

$$
D_{\bar{s}}^{(d)}\left(m ; \bar{\mu}_{1}, \bar{\mu}_{2}\right)=\int_{-\pi}^{\pi} e^{i \lambda m} \chi_{\bar{\mu}_{1}}^{(d)}\left(e^{-i \lambda}\right) \chi_{\bar{\mu}_{2}}^{(d)}\left(e^{i \lambda}\right) \frac{1}{\left|\beta^{(d)}(i \lambda)\right|^{2}} d F(\lambda),
$$

where $F(\lambda)$ is the matrix-valued spectral function of the stationary stochastic sequence $\chi_{\bar{\mu}, \bar{s}}^{(d)}(\vec{\xi}(m))$. The stationary GM increment sequence $\chi_{\bar{\mu}, \bar{s}}^{(d)}(\vec{\xi}(m))$ admits the spectral representation

$$
\chi_{\bar{\mu}, \bar{s}}^{(d)}(\vec{\xi}(m))=\int_{-\pi}^{\pi} e^{i m \lambda} \chi_{\bar{\mu}}^{(d)}\left(e^{-i \lambda}\right) \frac{1}{\beta^{(d)}(i \lambda)} d \vec{Z}_{\xi^{(d)}}(\lambda),
$$

where $\vec{Z}_{\xi^{(d)}}(\lambda)=\left\{Z_{p}(\lambda)\right\}_{p=1}^{T}$ is a (vector-valued) stochastic process with uncorrelated increments on $[-\pi, \pi)$ connected with the spectral function $F(\lambda)$ by the relation

$$
\begin{gathered}
\mathrm{E}\left(Z_{p}\left(\lambda_{2}\right)-Z_{p}\left(\lambda_{1}\right)\right)\left(\overline{Z_{q}\left(\lambda_{2}\right)-Z_{q}\left(\lambda_{1}\right)}\right)=F_{p q}\left(\lambda_{2}\right)-F_{p q}\left(\lambda_{1}\right), \\
-\pi \leq \lambda_{1}<\lambda_{2}<\pi, \quad p, q=1,2, \ldots, T .
\end{gathered}
$$

\subsection{Moving average representation of periodically stationary GM increment}

Denote by $H=L_{2}(\Omega, \mathcal{F}, \mathrm{P})$ the Hilbert space of random variables $\zeta$ with zero first moment, $\mathrm{E} \zeta=0$, finite second moment, $\mathrm{E}|\zeta|^{2}<\infty$, endowed with the inner product $\langle\zeta, \eta\rangle=\mathrm{E} \zeta \bar{\eta}$. Denote by $H\left(\vec{\xi}^{(d)}\right)$ the closed linear subspace of the space $H$ generated by components $\left\{\chi_{\bar{\mu}, \bar{s}}^{(d)}\left(\xi_{p}(m)\right), p=1, \ldots, T ; m \in \mathbb{Z}\right\}$ of the stationary stochastic GM increment sequence $\vec{\xi}^{(d)}=\left\{\chi_{\bar{\mu}, \bar{s}}^{(d)}\left(\xi_{p}(l)\right)\right\}_{p=1}^{T}, \bar{\mu}>\overline{0}$, and denote by $H^{q}\left(\vec{\xi}^{(d)}\right)$ the closed linear subspace generated by components $\left\{\chi_{\bar{\mu}, \bar{s}}^{(d)}\left(\xi_{p}(m)\right), p=1, \ldots, T ; m \leqslant q\right\}, q \in \mathbb{Z}$. Define a subspace

$$
S\left(\vec{\xi}^{(d)}\right)=\bigcap_{q \in \mathbb{Z}} H^{q}\left(\vec{\xi}^{(d)}\right)
$$

Stat., Optim. Inf. Comput. Vol. 8, September 2020 
of the Hilbert space $H\left(\vec{\xi}^{(d)}\right)$. Then the space $H\left(\vec{\xi}^{(d)}\right)$ admits a decomposition

$$
H\left(\vec{\xi}^{(d)}\right)=S\left(\vec{\xi}^{(d)}\right) \oplus R\left(\vec{\xi}^{(d)}\right),
$$

where $R\left(\vec{\xi}^{(d)}\right)$ is the orthogonal complement of the subspace $S\left(\vec{\xi}^{(d)}\right)$ in the space $H\left(\vec{\xi}^{(d)}\right)$.

Definition 2.6

A stationary (wide sense) stochastic GM increment sequence $\chi_{\bar{\mu}, \bar{s}}^{(d)}(\vec{\xi}(m))=\left\{\chi_{\bar{\mu}, \bar{s}}^{(d)}\left(\xi_{p}(m)\right)\right\}_{p=1}^{T}$ is called regular if $H\left(\vec{\xi}^{(d)}\right)=R\left(\vec{\xi}^{(d)}\right)$, and it is called singular if $H\left(\vec{\xi}^{(d)}\right)=S\left(\vec{\xi}^{(d)}\right)$.

Theorem 2.3

A stationary stochastic GM increment sequence $\chi_{\bar{\mu}, \bar{s}}^{(d)}(\vec{\xi}(m))=\left\{\chi_{\bar{\mu}, \bar{s}}^{(d)}\left(\xi_{p}(m)\right)\right\}_{p=1}^{T}$ is uniquely represented in the form

$$
\chi_{\bar{\mu}, \bar{s}}^{(d)}\left(\xi_{p}(m)\right)=\chi_{\bar{\mu}, \bar{s}}^{(d)}\left(\xi_{S, p}(m)\right)+\chi_{\bar{\mu}, \bar{s}}^{(d)}\left(\xi_{R, p}(m)\right),
$$

where $\chi_{\bar{\mu}, \bar{s}}^{(d)}\left(\xi_{R, p}(m)\right), p=1, \ldots, T$ is a regular stationary GM increment sequence and $\chi_{\bar{\mu}, \bar{s}}^{(d)}\left(\xi_{S, p}(m)\right), p=1, \ldots, T$ is a singular stationary GM increment sequence. The GM increment sequences $\chi_{\bar{\mu}, \bar{s}}^{(d)}\left(\xi_{R, p}(m)\right), p=1, \ldots, T$ and $\chi_{\bar{\mu}, \bar{s}}^{(d)}\left(\xi_{S, p}(m)\right), p=1, \ldots, T$ are orthogonal for all $m, k \in \mathbb{Z}$. They are defined by the formulas

$$
\begin{aligned}
\chi_{\bar{\mu}, \bar{s}}^{(d)}\left(\xi_{S, p}(m)\right) & =\mathrm{E}\left[\chi_{\bar{\mu}, \bar{s}}^{(d)}\left(\xi_{p}(m)\right) \mid S\left(\vec{\xi}^{(d)}\right)\right], \\
\chi_{\bar{\mu}, \bar{s}}^{(d)}\left(\xi_{R, p}(m)\right) & =\chi_{\bar{\mu}, \bar{s}}^{(d)}\left(\xi_{p}(m)\right)-\chi_{\bar{\mu}, \bar{s}}^{(d)}\left(\xi_{S, p}(m)\right), \quad p=1, \ldots, T .
\end{aligned}
$$

Consider an innovation sequence $\vec{\varepsilon}(u)=\left\{\varepsilon_{k}(u)\right\}_{k=1}^{q}, u \in \mathbb{Z}$ for a regular stationary GM increment $\chi_{\bar{\mu}, \bar{s}}^{(d)}\left(\xi_{R, p}(m)\right), p=1, \ldots, T$, namely, a sequence of uncorrelated random variables such that $\mathrm{E} \varepsilon_{k}(u) \bar{\varepsilon}_{j}(v)=$ $\delta_{k j} \delta_{u v}, \mathrm{E}\left|\varepsilon_{k}(u)\right|^{2}=1, k, j=1, \ldots, q ; u \in \mathbb{Z}$, and $H^{r}\left(\vec{\xi}^{(d)}\right)=H^{r}(\vec{\varepsilon})$ holds true for all $r \in \mathbb{Z}$, where $H^{r}(\vec{\varepsilon})$ is the Hilbert space generated by elements $\left\{\varepsilon_{k}(u): k=1, \ldots, q ; u \leq r\right\}, \delta_{k j}$ and $\delta_{u v}$ are Kronecker symbols.

Theorem 2.4

A stationary GM increment sequence $\chi_{\bar{\mu}, \bar{s}}^{(d)}(\vec{\xi}(m))$ is regular if and only if there exists an innovation sequence $\vec{\varepsilon}(u)=\left\{\varepsilon_{k}(u)\right\}_{k=1}^{q}, u \in \mathbb{Z}$ and a sequence of matrix-valued functions $\varphi^{(d)}(k, \bar{\mu})=\left\{\varphi_{i j}^{(d)}(k, \bar{\mu})\right\}_{i=\overline{1, T}}^{j=\overline{1, q}}, k \geq 0$, such that

$$
\sum_{k=0}^{\infty} \sum_{i=1}^{T} \sum_{j=1}^{q}\left|\varphi_{i j}^{(d)}(k, \bar{\mu})\right|^{2}<\infty, \quad \chi_{\bar{\mu}, \bar{s}}^{(d)}(\vec{\xi}(m))=\sum_{k=0}^{\infty} \varphi^{(d)}(k, \bar{\mu}) \vec{\varepsilon}(m-k) .
$$

Representation (17) is called the canonical moving average representation of the stochastic stationary GM increment sequence $\chi_{\bar{\mu}, \bar{s}}^{(d)}(\vec{\xi}(m))$.

The spectral function $F(\lambda)$ of a stationary GM increment sequence $\chi_{\bar{\mu}, \bar{s}}^{(d)}(\vec{\xi}(m))$ which admits the canonical representation (17) has the spectral density $f(\lambda)=\left\{f_{i j}(\lambda)\right\}_{i, j=1}^{T}$ admitting the canonical factorization

$$
f(\lambda)=\Phi\left(e^{-i \lambda}\right) \Phi^{*}\left(e^{-i \lambda}\right),
$$

where the function $\Phi(z)=\sum_{k=0}^{\infty} \varphi(k) z^{k}$ has analytic in the unit circle $\{z:|z| \leq 1\}$ components $\Phi_{i j}(z)=$ $\sum_{k=0}^{\infty} \varphi_{i j}(k) z^{k} ; i=1, \ldots, T ; j=1, \ldots, q$. Based on moving average representation (17) define

$$
\Phi_{\bar{\mu}}(z)=\sum_{k=0}^{\infty} \varphi^{(d)}(k, \bar{\mu}) z^{k}=\sum_{k=0}^{\infty} \varphi_{\bar{\mu}}(k) z^{k} .
$$

Then the following relation holds true:

$$
\Phi_{\bar{\mu}}\left(e^{-i \lambda}\right) \Phi_{\bar{\mu}}^{*}\left(e^{-i \lambda}\right)=\frac{\left|\chi_{\bar{\mu}}^{(d)}\left(e^{-i \lambda}\right)\right|^{2}}{\left|\beta^{(d)}(i \lambda)\right|^{2}} f(\lambda)=\prod_{j=1}^{r} \frac{\left|1-e^{-i \lambda \mu_{j} s_{j}}\right|^{2 d_{j}}}{\prod_{k_{j}=-\left[s_{j} / 2\right]}^{\left[s_{j} / 2\right]}\left|\lambda-2 \pi k_{j} / s_{j}\right|^{2 d_{j}}} f(\lambda) .
$$

We will use the one-sided moving average representation (17) and relation (19) for finding the mean square optimal estimates of unobserved values of vector-valued sequences with stationary GM increments. 


\section{Hilbert space projection method of forecasting}

\subsection{Forecasting of vector-valued stationary GM increment}

Consider a vector-valued stochastic sequence with stationary GM increments $\vec{\xi}(m)$ constructed from the sequence $\zeta(m)$ with the help of transformation (13). Let the stationary GM increment sequence $\chi_{\bar{\mu}, \bar{s}}^{(d)}(\vec{\xi}(m))=$ $\left\{\chi_{\bar{\mu}, \bar{s}}^{(d)}\left(\xi_{p}(m)\right)\right\}_{p=1}^{T}$ has an absolutely continuous spectral function $F(\lambda)$ and the spectral density $f(\lambda)=$ $\left\{f_{i j}(\lambda)\right\}_{i, j=1}^{T}$. Without loss of generality we will assume that $\mathrm{E} \chi_{\bar{\mu}, \bar{s}}^{(d)}(\vec{\xi}(m))=0$ and $\bar{\mu}>\overline{0}$.

Consider the problem of mean square optimal linear estimation of the functionals

$$
A \vec{\xi}=\sum_{k=0}^{\infty}(\vec{a}(k))^{\top} \vec{\xi}(k), \quad A_{N} \vec{\xi}=\sum_{k=0}^{N}(\vec{a}(k))^{\top} \vec{\xi}(k),
$$

which depend on unobserved values of the stochastic sequence $\vec{\xi}(k)=\left\{\xi_{p}(k)\right\}_{p=1}^{T}$ with stationary GM increments. Estimates are based on observations of the sequence $\vec{\xi}(k)$ at points $k=-1,-2, \ldots$.

We will suppose that the following conditions are satisfied:

- conditions on coefficients $\vec{a}(k)=\left\{a_{p}(k)\right\}_{p=1}^{T}, k \geq 0$, and a linear transformation $D^{\bar{\mu}}$ to be defined in Lemma 3.1

$$
\begin{array}{cl}
\sum_{k=0}^{\infty}\|\vec{a}(k)\|<\infty, & \sum_{k=0}^{\infty}(k+1)\|\vec{a}(k)\|^{2}<\infty, \\
\sum_{k=0}^{\infty}\left\|\left(D^{\bar{\mu}} \mathbf{a}\right)_{k}\right\|<\infty, & \sum_{k=0}^{\infty}(k+1)\left\|\left(D^{\bar{\mu}} \mathbf{a}\right)_{k}\right\|^{2}<\infty,
\end{array}
$$

- the minimality condition on the spectral density $f(\lambda)$

$$
\int_{-\pi}^{\pi} \operatorname{Tr}\left[\frac{\left|\beta^{(d)}(i \lambda)\right|^{2}}{\left|\chi_{\bar{\mu}}^{(d)}\left(e^{-i \lambda}\right)\right|^{2}} f^{-1}(\lambda)\right] d \lambda<\infty .
$$

The latter one is the necessary and sufficient condition under which the mean square errors of estimates of functionals $A \vec{\xi}$ and $A_{N} \vec{\xi}$ are not equal to 0 .

The classical Hilbert space estimation technique proposed by Kolmogorov [28] can be described as a 3-stage procedure: (i) define a target element of the space $H=L_{2}(\Omega, \mathcal{F}, \mathrm{P})$ to be estimated, (ii) define a subspace of $H$ generated by observations, (iii) find an estimate of the target element as an orthogonal projection on the defined subspace.

Stage i. Neither functional $A \vec{\xi}$ nor $A_{N} \vec{\xi}$ belongs to the space $H$. With the help of the following lemma and the corresponding corollary, we describe representations of these functionals as sums of functionals with finite second moments belonging to $H$ and functionals depending on observed values of the sequence $\vec{\xi}(k)$ ("initial values").

Lemma 3.1

The functional $A \vec{\xi}$ admits a representation

$$
A \vec{\xi}=B \chi \vec{\xi}-V \vec{\xi}
$$

where

$$
\begin{gathered}
B \chi \vec{\xi}=\sum_{k=0}^{\infty}(\vec{b}(k))^{\top} \chi_{\bar{\mu}, \bar{s}}^{(d)}(\vec{\xi}(k)), \quad V \vec{\xi}=\sum_{k=-n(\gamma)}^{-1}(\vec{v}(k))^{\top} \vec{\xi}(k), \\
\vec{v}(k)=\sum_{l=0}^{k+n(\gamma)} \operatorname{diag}_{T}\left(e_{\nu}(l-k)\right) \vec{b}(l), \quad k=-1,-2, \ldots,-n(\gamma), \\
\vec{b}(k)=\sum_{m=k}^{\infty} \operatorname{diag}_{T}\left(d_{\bar{\mu}}(m-k)\right) \vec{a}(m)=\left(D^{\bar{\mu}} \mathbf{a}\right)_{k}, \quad k=0,1,2, \ldots,
\end{gathered}
$$


$\vec{b}(k)=\left(b_{1}(k), b_{2}(k), \ldots, b_{T}(k)\right)^{\top}, \mathbf{a}=\left((\vec{a}(0))^{\top},(\vec{a}(1))^{\top},(\vec{a}(2))^{\top}, \ldots\right)^{\top}, \vec{v}(k)=\left(v_{1}(k), v_{2}(k), \ldots, v_{T}(k)\right)^{\top}, D^{\bar{\mu}}$ is a linear transformation determined by a matrix with $T \times T$ entries $D^{\bar{\mu}}(k, j), k, j=0,1,2, \ldots$ such that $D^{\bar{\mu}}(k, j)=\operatorname{diag}_{T}\left(d_{\bar{\mu}}(j-k)\right)$ if $0 \leq k \leq j$ and $D^{\bar{\mu}}(k, j)=\operatorname{diag}_{T}(0)$ for $0 \leq j<k ; \operatorname{diag}_{T}(x)$ denotes a $T \times T$ diagonal matrix with the entry $x$ on its diagonal, coefficients $\left\{d_{\bar{\mu}}(k): k \geq 0\right\}$ are determined by the relationship

$$
\sum_{k=0}^{\infty} d_{\bar{\mu}}(k) x^{k}=\prod_{i=1}^{r}\left(\sum_{j_{i}=0}^{\infty} x^{\mu_{i} s_{i} j_{i}}\right)^{d_{i}} .
$$

Proof

See Appendix.

Corollary 3.1

The functional $A_{N} \vec{\xi}$ allows a representation

$$
\begin{gathered}
A_{N} \vec{\xi}=B_{N} \chi \vec{\xi}-V_{N} \vec{\xi}, \\
B_{N} \chi \vec{\xi}=\sum_{k=0}^{N}\left(\vec{b}_{N}(k)\right)^{\top} \chi_{\bar{\mu}, \bar{s}}^{(d)}(\vec{\xi}(k)), \quad V_{N} \vec{\xi}=\sum_{k=-n(\gamma)}^{-1}\left(\vec{v}_{N}(k)\right)^{\top} \vec{\xi}(k),
\end{gathered}
$$

where the coefficients $\quad \vec{b}_{N}(k)=\left\{b_{N, p}(k)\right\}_{p=1}^{T}, k=0,1, \ldots, N \quad$ and $\quad \vec{v}_{N}(k)=\left\{v_{N, p}(k)\right\}_{p=1}^{T}, k=$ $-1,-2, \ldots,-n(\gamma)$ are calculated by the formulas

$$
\begin{aligned}
& \vec{v}_{N}(k)=\sum_{l=0}^{N \wedge k+n(\gamma)} \operatorname{diag}_{T}\left(e_{\nu}(l-k)\right) \vec{b}_{N}(l), k=-1,-2, \ldots,-n(\gamma), \\
& \vec{b}_{N}(k)=\sum_{m=k}^{N} \operatorname{diag}_{T}\left(d_{\bar{\mu}}(m-k)\right) \vec{a}(m)=\left(D_{N}^{\bar{\mu}} \mathbf{a}_{N}\right)_{k}, k=0,1, \ldots, N,
\end{aligned}
$$

$D_{N}^{\bar{\mu}}$ is the linear transformation determined by an infinite matrix with the entries $\left(D_{N}^{\bar{\mu}}\right)(k, j)=\operatorname{diag}_{T}\left(d_{\bar{\mu}}(j-k)\right)$ if $0 \leq k \leq j \leq N$, and $\left(D_{N}^{\bar{\mu}}\right)(k, j)=0$ if $j<k$ or $j, k>N$; $\mathbf{a}_{N}=\left((\vec{a}(0))^{\top},(\vec{a}(1))^{\top}, \ldots,(\vec{a}(N))^{\top}, \overrightarrow{0} \ldots\right)^{\top}$.

So that, Lemma 3.1 provides a representation of the functional $A \vec{\xi}$ as a sum of an element $B \chi \vec{\xi}$ from the space $H=L_{2}(\Omega, \mathcal{F}, \mathrm{P})$ under conditions (21) - (22) and linear combination $V \vec{\xi}$ of a finite number of initial values $\vec{\xi}(k)$, $k=-1,-2, \ldots,-n(\gamma)$, which are observed. Thus, the following equality hold true

$$
\widehat{A} \vec{\xi}=\widehat{B} \chi \vec{\xi}-V \vec{\xi}
$$

Denote by $\Delta(f, \widehat{A} \vec{\xi}):=\mathrm{E}|A \vec{\xi}-\widehat{A} \vec{\xi}|^{2}$ the mean square error of the optimal estimate $\widehat{A} \vec{\xi}$ of the functional $A \vec{\xi}$ and let $\Delta(f, \widehat{B} \chi \vec{\xi}):=\mathrm{E}|B \chi \vec{\xi}-\widehat{B} \chi \vec{\xi}|^{2}$ denote the mean square error of the optimal estimate $\widehat{B} \chi \vec{\xi}$ of the functional $B \chi \vec{\xi}$. Then

$$
\Delta(f ; \widehat{A} \vec{\xi})=\mathrm{E}|A \vec{\xi}-\widehat{A} \vec{\xi}|^{2}=\mathrm{E}|B \chi \vec{\xi}-V \vec{\xi}-\widehat{B} \chi \vec{\xi}+V \vec{\xi}|^{2}=\mathrm{E}|B \chi \vec{\xi}-\widehat{B} \chi \vec{\xi}|^{2}=\Delta(f ; \widehat{B} \chi \vec{\xi})
$$

Thus, we have defined the functional $B \chi \vec{\xi}$ to be used in finding the optimal linear estimate of the functional $A \vec{\xi}$.

At stage ii, we recall the subspace $H^{0-}\left(\vec{\xi}^{(d)}\right):=H^{-1}\left(\vec{\xi}^{(d)}\right)$ of the Hilbert space $H=L_{2}(\Omega, \mathcal{F}, \mathrm{P})$ defined in Subsection 2.2, which is generated by observations $\left\{\chi_{\bar{\mu}, \bar{s}}^{(d)}\left(\xi_{p}(k)\right), p=1, \ldots, T ; k \leq-1\right\}$. Denote by $L_{2}^{0-}(f)$ the closed linear subspace of the Hilbert space $L_{2}(f)$ of vector-valued functions endowed with the inner product $\left\langle g_{1} ; g_{2}\right\rangle=\int_{-\pi}^{\pi}\left(g_{1}(\lambda)\right)^{\top} f(\lambda) \overline{g_{2}(\lambda)} d \lambda$ which is generated by functions

$$
e^{i \lambda k} \chi_{\bar{\mu}}^{(d)}\left(e^{-i \lambda}\right)\left(\beta^{(d)}(i \lambda)\right)^{-1} \boldsymbol{\delta}_{l}, \quad \boldsymbol{\delta}_{l}=\left\{\delta_{l p}\right\}_{p=1}^{T}, l=1, \ldots, T ; k \leqslant-1,
$$


where $\delta_{l p}$ are Kronecker symbols. The relation

$$
\chi_{\bar{\mu}, \bar{s}}^{(d)}\left(\xi_{p}(m)\right)=\int_{-\pi}^{\pi} e^{i \lambda m} \chi_{\bar{\mu}}^{(d)}\left(e^{-i \lambda}\right) \frac{1}{\beta^{(d)}(i \lambda)} d Z_{p}(\lambda), \quad p=1,2, \ldots, T,
$$

implies a relation between elements $\chi_{\bar{\mu}, \bar{s}}^{(d)}\left(\xi_{p}(m)\right)$ of the space $H\left(\vec{\xi}^{(d)}\right)$ and elements $e^{i \lambda m} \chi_{\bar{\mu}}^{(d)}\left(e^{-i \lambda}\right)\left(\beta^{(d)}(i \lambda)\right)^{-1} \boldsymbol{\delta}_{p}$ of the space $L_{2}(f)$. The spectral representation of the functional $B \chi \vec{\xi}$ can be written in the form

$$
B \chi \vec{\xi}=\int_{-\pi}^{\pi}\left(\vec{B}_{\bar{\mu}}\left(e^{i \lambda}\right)\right)^{\top} \frac{\chi_{\bar{\mu}}^{(d)}\left(e^{-i \lambda}\right)}{\beta^{(d)}(i \lambda)} d \vec{Z}_{\xi^{(d)}}(\lambda),
$$

where

$$
\vec{B}_{\bar{\mu}}\left(e^{i \lambda}\right)=\sum_{k=0}^{\infty} \vec{b}(k) e^{i \lambda k}=\sum_{k=0}^{\infty}\left(D^{\bar{\mu}} \mathbf{a}\right)_{k} e^{i \lambda k}
$$

Thus, at stage iii, the problem is equivalent to finding a projection of the element $\vec{B}_{\bar{\mu}}\left(e^{i \lambda}\right) \frac{\chi_{\bar{\mu}}^{(d)}\left(e^{-i \lambda}\right)}{\beta^{(d)}(i \lambda)}$ of the Hilbert space $L_{2}(f)$ on the subspace $L_{2}^{0-}(f)$.

Relation (26) implies that every linear estimate $\widehat{A} \vec{\xi}$ of the functional $A \vec{\xi}$ can be written in the form

$$
\widehat{A} \vec{\xi}=\int_{-\pi}^{\pi}\left(\vec{h}_{\bar{\mu}}(\lambda)\right)^{\top} d \vec{Z}_{\xi^{(d)}}(\lambda)-\sum_{k=-n(\nu)}^{-1}(\vec{v}(k))^{\top} \vec{\xi}(k),
$$

where $\vec{h}_{\bar{\mu}}(\lambda)=\left\{h_{p}(\lambda)\right\}_{p=1}^{T}$ is the spectral characteristic of the estimate $\widehat{B} \vec{\xi}$, which is a projection of the element $\vec{B}_{\bar{\mu}}\left(e^{i \lambda}\right) \frac{\chi_{\bar{\mu}}^{(d)}\left(e^{-i \lambda}\right)}{\beta^{(d)}(i \lambda)}$ on the subspace $L_{2}^{0-}(f)$. This estimate is characterized by the following conditions:

$$
\begin{gathered}
\vec{h}_{\bar{\mu}}(\lambda) \in L_{2}^{0-}(f), \\
\left(\vec{B}_{\bar{\mu}}\left(e^{i \lambda}\right) \frac{\chi_{\bar{\mu}}^{(d)}\left(e^{-i \lambda}\right)}{\beta^{(d)}(i \lambda)}-\vec{h}_{\bar{\mu}}(\lambda)\right) \perp L_{2}^{0-}(f) .
\end{gathered}
$$

Condition (30) implies the following relation holding true for all $k \leqslant-1$

$$
\int_{-\pi}^{\pi}\left(\vec{B}_{\bar{\mu}}\left(e^{i \lambda}\right) \frac{\chi_{\bar{\mu}}^{(d)}\left(e^{-i \lambda}\right)}{\beta^{(d)}(i \lambda)}-\vec{h}_{\bar{\mu}}(\lambda)\right)^{\top} f(\lambda) e^{-i \lambda k} \frac{\chi_{\bar{\mu}}^{(d)}\left(e^{i \lambda}\right)}{\bar{\beta}^{(d)}(i \lambda)} d \lambda=\overrightarrow{0} .
$$

Thus, the spectral characteristic of the estimate $\widehat{B} \chi \vec{\xi}$ can be represented in the form

$$
\left(\vec{h}_{\bar{\mu}}(\lambda)\right)^{\top}=\left(\vec{B}_{\bar{\mu}}\left(e^{i \lambda}\right)\right)^{\top} \frac{\chi_{\bar{\mu}}^{(d)}\left(e^{-i \lambda}\right)}{\beta^{(d)}(i \lambda)}-\frac{\overline{\beta^{(d)}(i \lambda)}}{\chi_{\bar{\mu}}^{(d)}\left(e^{i \lambda}\right)}\left(\vec{C}_{\bar{\mu}}\left(e^{i \lambda}\right)\right)^{\top} f^{-1}(\lambda),
$$

where

$$
\vec{C}_{\bar{\mu}}\left(e^{i \lambda}\right)=\sum_{k=0}^{\infty} \vec{c}_{\bar{\mu}}(k) e^{i \lambda k}
$$

and $\vec{c}(k)=\left\{c_{p}(k)\right\}_{p=1}^{T}, k \geqslant 0$ are unknown coefficients to be found.

Condition (29) implies the following representation of the spectral characteristic $\vec{h}_{\bar{\mu}}(\lambda)$

$$
\vec{h}_{\bar{\mu}}(\lambda)=\vec{h}(\lambda) \chi_{\bar{\mu}}^{(d)}\left(e^{-i \lambda}\right) \frac{1}{\beta^{(d)}(i \lambda)}, \quad \vec{h}(\lambda)=\sum_{k=1}^{\infty} \vec{s}(k) e^{-i \lambda k},
$$


which allows us to write the relations

$$
\int_{-\pi}^{\pi}\left[\left(\vec{B}_{\bar{\mu}}\left(e^{i \lambda}\right)\right)^{\top}-\frac{\left|\beta^{(d)}(i \lambda)\right|^{2}}{\chi_{\bar{\mu}}^{(d)}\left(e^{-i \lambda}\right) \chi_{\bar{\mu}}^{(d)}\left(e^{i \lambda}\right)}\left(\vec{C}_{\bar{\mu}}\left(e^{i \lambda}\right)\right)^{\top} f^{-1}(\lambda)\right] e^{-i j \lambda} d \lambda=\overrightarrow{0}, j \geqslant 0 .
$$

Next we define the matrix-valued Fourier coefficients

$$
F^{\bar{\mu}}(k, j)=\frac{1}{2 \pi} \int_{-\pi}^{\pi} e^{i \lambda(j-k)} \frac{\left|\beta^{(d)}(i \lambda)\right|^{2}}{\left|\chi_{\bar{\mu}}^{(d)}\left(e^{-i \lambda}\right)\right|^{2}} f^{-1}(\lambda) d \lambda, \quad k, j \geq 0,
$$

and rewrite relation (32) as a system of linear vector equations

$$
\vec{b}(j)=\sum_{k=0}^{\infty} F^{\bar{\mu}}(j, k) \vec{c}_{\bar{\mu}}(k), \quad j \geq 0,
$$

determining the unknown coefficients $\vec{c}_{\bar{\mu}}(k), k \geq 0$. This system can be presented in the matrix form

$$
D^{\bar{\mu}} \mathbf{a}=\mathbf{F}_{\bar{\mu}} \mathbf{c}_{\bar{\mu}},
$$

where

$$
\mathbf{c}_{\bar{\mu}}=\left(\left(\vec{c}_{\bar{\mu}}(0)\right)^{\top},\left(\vec{c}_{\bar{\mu}}(1)\right)^{\top},\left(\vec{c}_{\bar{\mu}}(2)\right)^{\top}, \ldots\right)^{\top}, \mathbf{a}=\left((\vec{a}(0))^{\top},(\vec{a}(1))^{\top},(\vec{a}(2))^{\top}, \ldots\right)^{\top},
$$

$\mathbf{F}_{\bar{\mu}}$ is a linear operator in the space $\ell_{2}$ which is determined by a matrix with the $T \times T$ matrix entries $\mathbf{F}_{\bar{\mu}}(j, k)=$ $F^{\bar{\mu}}(j, k), j, k \geq 0$; the linear transformation $D^{\bar{\mu}}$ is defined in Lemma 3.1.

To show that operator $\mathbf{F}_{\bar{\mu}}$ is invertible we note that the problem of projection of the element $B \vec{\xi}$ of the Hilbert space $H$ on the closed convex set $H^{0-}\left(\vec{\xi}_{\bar{\mu}}^{(d)}\right)$ has a unique solution for each non-zero coefficients $\{\vec{a}(0), \vec{a}(1)), \vec{a}(2), \ldots\}$, satisfying conditions (21) - (22). Therefore, equation (34) has a unique solution for each vector $D^{\bar{\mu}} \mathbf{a}$, which implies existence of the inverse operator $\mathbf{F}_{\bar{\mu}}^{-1}$.

Therefore, coefficients $\vec{c}_{\bar{\mu}}(k), k \geq 0$, which determine the spectral characteristic $\vec{h}_{\bar{\mu}}(\lambda)$, can be calculated as

$$
\vec{c}_{\mu}(k)=\left(\mathbf{F}_{\bar{\mu}}^{-1} D^{\bar{\mu}} \mathbf{a}\right)_{k}, \quad k \geq 0,
$$

where $\left(\mathbf{F}_{\bar{\mu}}^{-1} D^{\bar{\mu}} \mathbf{a}\right)_{k}, k \geq 0$, is the $k$ th $T$-dimension vector element of the vector $\mathbf{F}_{\bar{\mu}}^{-1} D^{\bar{\mu}} \mathbf{a}$.

The spectral characteristic $\vec{h}_{\bar{\mu}}(\lambda)$ of the estimate $\widehat{B} \chi \vec{\xi}$ is calculated by the formula

$$
\left(\vec{h}_{\bar{\mu}}(\lambda)\right)^{\top}=\left(\vec{B}_{\bar{\mu}}\left(e^{i \lambda}\right)\right)^{\top} \frac{\chi_{\bar{\mu}}^{(d)}\left(e^{-i \lambda}\right)}{\beta^{(d)}(i \lambda)}-\frac{\overline{\beta^{(d)}(i \lambda)}}{\chi_{\bar{\mu}}^{(d)}\left(e^{i \lambda}\right)}\left(\sum_{k=0}^{\infty}\left(\mathbf{F}_{\bar{\mu}}^{-1} D^{\bar{\mu}} \mathbf{a}\right)_{k} e^{i \lambda k}\right)^{\top} f^{-1}(\lambda) .
$$

The value of the mean square error of the estimate $\widehat{A} \vec{\xi}$ is calculated by the formula

$$
\begin{aligned}
& \Delta(f ; \widehat{A} \vec{\xi})=\Delta(f ; \widehat{B} \chi \vec{\xi})=\mathrm{E}|B \chi \vec{\xi}-\widehat{B} \chi \vec{\xi}|^{2} \\
& =\frac{1}{2 \pi} \int_{-\pi}^{\pi} \frac{\overline{\beta^{(d)}(i \lambda)}}{\chi_{\bar{\mu}}^{(d)}\left(e^{i \lambda}\right)}\left(\sum_{k=0}^{\infty}\left(\mathbf{F}_{\bar{\mu}}^{-1} D^{\bar{\mu}} \mathbf{a}\right)_{k} e^{i \lambda k}\right)^{\top} f(\lambda)\left(\overline{\sum_{k=0}^{\infty}\left(\mathbf{F}_{\bar{\mu}}^{-1} D^{\bar{\mu}} \mathbf{a}\right)_{k} e^{i \lambda k}}\right) \frac{\beta^{(d)}(i \lambda)}{\chi_{\bar{\mu}}^{(d)}\left(e^{-i \lambda}\right)} d \lambda \\
& =\left\langle D^{\bar{\mu}} \mathbf{a}, \mathbf{F}_{\bar{\mu}}^{-1} D^{\bar{\mu}} \mathbf{a}\right\rangle \text {. }
\end{aligned}
$$

Next consider the problem in the case where the GM incremental sequence of the stochastic sequence $\vec{\xi}(m)$ admits moving-average representation (17) and its spectral density $f(\lambda)=\left\{f_{i j}(\lambda)\right\}_{i, j=1}^{T}$ admits the canonical factorization (18), (19), namely

$$
f(\lambda)=\Phi\left(e^{-i \lambda}\right) \Phi^{*}\left(e^{-i \lambda}\right), \quad \frac{\left|\chi_{\bar{\mu}}^{(d)}\left(e^{-i \lambda}\right)\right|^{2}}{\left|\beta^{(d)}(i \lambda)\right|^{2}} f(\lambda)=\Phi_{\bar{\mu}}\left(e^{-i \lambda}\right) \Phi_{\bar{\mu}}^{*}\left(e^{-i \lambda}\right),
$$


where

$$
\Phi\left(e^{-i \lambda}\right)=\sum_{k=0}^{\infty} \varphi(k) e^{-i \lambda k}, \quad \Phi_{\bar{\mu}}\left(e^{-i \lambda}\right)=\sum_{k=0}^{\infty} \varphi_{\bar{\mu}}(k) e^{-i \lambda k},
$$

and $\varphi_{\bar{\mu}}(k)=\left\{\varphi_{i j}(k)\right\}_{i=\overline{1, T}}^{j=\overline{1, q}}, k=0,1,2, \ldots$ Let $E_{q}$ denote the identity $q \times q$ matrix. Define the matrix-valued function $\Psi_{\bar{\mu}}\left(e^{-i \lambda}\right)=\left\{\Psi_{i j}\left(e^{-i \lambda}\right)\right\}_{i=\overline{1, q}}^{j=\overline{1, T}}$ by the equation

$$
\Psi_{\bar{\mu}}\left(e^{-i \lambda}\right) \Phi_{\bar{\mu}}\left(e^{-i \lambda}\right)=E_{q} .
$$

Formulas for calculating the spectral characteristic $\vec{h}_{\bar{\mu}}(\lambda)$ and the value of the mean square error $\Delta(f ; \widehat{A} \vec{\xi})$ of the estimate $\widehat{A} \vec{\xi}$ can be presented in terms of the function $\Psi_{\bar{\mu}}\left(e^{-i \lambda}\right)$ and the factorization coefficients $\varphi_{\bar{\mu}}(k)$, $k=0,1,2, \ldots$. One can directly check that conditions (29) and (30) are satisfied by the function

$$
\vec{h}_{\bar{\mu}}(\lambda)=\frac{\chi_{\bar{\mu}}^{(d)}\left(e^{-i \lambda}\right)}{\beta^{(d)}(i \lambda)}\left(\vec{B}_{\mu}\left(e^{i \lambda}\right)-\left(\Psi_{\bar{\mu}}\left(e^{-i \lambda}\right)\right)^{\top} \vec{r}_{\bar{\mu}}\left(e^{i \lambda}\right)\right),
$$

where

$$
\begin{aligned}
\vec{r}_{\bar{\mu}}\left(e^{i \lambda}\right) & =\sum_{k=0}^{\infty}\left(D^{\bar{\mu}} \mathbf{A} \varphi_{\bar{\mu}}\right)_{k} e^{i \lambda k} \\
\left(D^{\bar{\mu}} \mathbf{A} \varphi_{\bar{\mu}}\right)_{k} & =\sum_{m=0}^{\infty} \sum_{l=m}^{\infty}\left(\varphi_{\bar{\mu}}(m)\right)^{\top} D^{\bar{\mu}}(m, l) \vec{a}(l+k)=\sum_{m=0}^{\infty} \sum_{l=k}^{\infty}\left(\varphi_{\bar{\mu}}(m)\right)^{\top} \vec{a}(m+l) d_{\bar{\mu}}(l-k),
\end{aligned}
$$

and $\mathbf{A}$ is a linear symmetric operator which is determined by a matrix with the entries $\mathbf{A}(k, j)=\vec{a}(k+j), k, j \geq 0$. The defined operators $D^{\bar{\mu}} \mathbf{A}$ and $\mathbf{A}$ are compact under conditions (21) - (22). Then the value of the mean square error is calculated by the formula

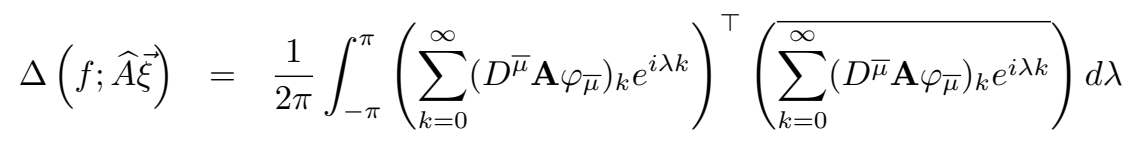

$$
\begin{aligned}
& =\frac{1}{2 \pi} \int_{-\pi}^{\pi}\left\|\vec{r}_{\bar{\mu}}\left(e^{i \lambda}\right)\right\|^{2} d \lambda=\left\|D^{\bar{\mu}} \mathbf{A} \varphi_{\bar{\mu}}\right\|^{2} .
\end{aligned}
$$

The derived results are summarized in the following theorem.

Theorem 3.1

Let a vector-valued stochastic sequence $\{\vec{\xi}(m), m \in \mathbb{Z}\}$ determine a stationary stochastic GM increment sequence $\chi_{\bar{\mu}, \bar{s}}^{(n)}(\vec{\xi}(m))$ with the spectral density matrix $f(\lambda)=\left\{f_{i j}(\lambda)\right\}_{i, j=1}^{T}$ which satisfies the minimality condition (23). Let coefficients $\vec{a}(j), j \geqslant 0$ satisfy conditions (21) - (22).

Then the optimal linear estimate $\widehat{A} \vec{\xi}$ of the functional $A \vec{\xi}$ based on observations of the sequence $\vec{\xi}(m)$ at points $m=-1,-2, \ldots$ is calculated by formula (28). The spectral characteristic $\vec{h}_{\bar{\mu}}(\lambda)=\left\{h_{p}(\lambda)\right\}_{p=1}^{T}$ and the value of the mean square error $\Delta(f ; \widehat{A} \vec{\xi})$ of the estimate $\widehat{A} \vec{\xi}$ are calculated by formulas (36) and (37) respectively.

In the case where the spectral density $f(\lambda)$ admits the canonical factorization (38) the spectral characteristic and the value of the mean square error of the optimal estimate $\widehat{A} \xi$ can be calculated by formulas (39) and (40) respectively.

\subsection{Estimates of functional $A_{N} \vec{\xi}$ and value $\xi_{p}(N)$}

Theorem 3.1 allows us to find the optimal estimate $\widehat{A}_{N} \vec{\xi}$ of the functional $A_{N} \vec{\xi}$ which depends on the unobserved values $\vec{\xi}(m), m=0,1,2, \ldots, N$, based on observations of the sequence $\vec{\xi}(m)$ at points $m=-1,-2, \ldots$ Put $\vec{a}(k)=0$ for $k>N$. Then we get that the spectral characteristic $\vec{h}_{\mu, N}(\lambda)$ of the optimal estimate 


$$
\widehat{A}_{N} \vec{\xi}=\int_{-\pi}^{\pi}\left(\vec{h}_{\bar{\mu}, N}(\lambda)\right)^{\top} d \vec{Z}_{\xi^{(d)}}(\lambda)-\sum_{k=-n(\gamma)}^{-1}\left(\vec{v}_{N}(k)\right)^{\top} \vec{\xi}(k),
$$

is calculated by the formula

$$
\left(\vec{h}_{\bar{\mu}, N}(\lambda)\right)^{\top}=\left(\vec{B}_{\bar{\mu}, N}\left(e^{i \lambda}\right)\right)^{\top} \frac{\chi_{\bar{\mu}}^{(d)}\left(e^{-i \lambda}\right)}{\beta^{(d)}(i \lambda)}-\frac{\overline{\beta^{(d)}(i \lambda)}}{\chi_{\bar{\mu}}^{(d)}\left(e^{i \lambda}\right)}\left(\sum_{k=0}^{\infty}\left(\mathbf{F}_{\bar{\mu}}^{-1} D_{N}^{\bar{\mu}} \mathbf{a}_{N}\right)_{k} e^{i \lambda k}\right)^{\top} f^{-1}(\lambda) .
$$

where

$$
B_{\bar{\mu}, N}\left(e^{i \lambda}\right)=\sum_{k=0}^{N}\left(D_{N}^{\bar{\mu}} \mathbf{a}_{N}\right)_{k} e^{i \lambda k},
$$

and $D_{N}^{\bar{\mu}}$ is defined in Corollary 3.1. The value of the mean square error of the estimate $\widehat{A}_{N} \xi$ is

$$
\begin{aligned}
& \Delta\left(f, \widehat{A}_{N} \vec{\xi}\right)=\Delta\left(f, \widehat{B}_{N} \chi \vec{\xi}\right)=\mathrm{E}\left|B_{N} \chi \vec{\xi}-\widehat{B}_{N} \chi \vec{\xi}\right|^{2} \\
& =\frac{1}{2 \pi} \int_{-\pi}^{\pi} \frac{\overline{\beta^{(d)}(i \lambda)}}{\chi_{\bar{\mu}}^{(d)}\left(e^{i \lambda}\right)}\left(\sum_{k=0}^{\infty}\left(\mathbf{F}_{\bar{\mu}}^{-1} D_{N}^{\bar{\mu}} \mathbf{a}_{N}\right)_{k} e^{i \lambda k}\right)^{\top} f(\lambda)\left(\overline{\left.\sum_{k=0}^{\infty}\left(\mathbf{F}_{\bar{\mu}}^{-1} D_{N}^{\bar{\mu}} \mathbf{a}_{N}\right)_{k} e^{i \lambda k}\right)} \frac{\beta^{(d)}(i \lambda)}{\chi_{\bar{\mu}}^{(d)}\left(e^{-i \lambda}\right)} d \lambda\right. \\
& =\left\langle D_{N}^{\bar{\mu}} \mathbf{a}_{N}, \mathbf{F}_{\bar{\mu}}^{-1} D_{N}^{\bar{\mu}} \mathbf{a}_{N}\right\rangle \text {. }
\end{aligned}
$$

In the case where the spectral density $f(\lambda)$ admits the canonical factorization (38) the spectral characteristic can be calculated as

$$
\vec{h}_{\bar{\mu}, N}(\lambda)=\frac{\chi_{\bar{\mu}}^{(d)}\left(e^{-i \lambda}\right)}{\beta^{(d)}(i \lambda)}\left(\vec{B}_{\bar{\mu}, N}\left(e^{i \lambda}\right)-\left(\Psi_{\bar{\mu}}\left(e^{-i \lambda}\right)\right)^{\top} \vec{r}_{\bar{\mu}, N}\left(e^{i \lambda}\right)\right)
$$

where

$$
\vec{r}_{\bar{\mu}, N}\left(e^{i \lambda}\right)=\sum_{k=0}^{N}\left(\widetilde{D}_{N}^{\bar{\mu}} \mathbf{A}_{N} \varphi_{\bar{\mu}, N}\right)_{k} e^{i \lambda k}, \quad\left(\widetilde{D}_{N}^{\bar{\mu}} \mathbf{A}_{N} \varphi_{\bar{\mu}, N}\right)_{k}=\sum_{m=0}^{N} \sum_{l=k}^{N}\left(\varphi_{\bar{\mu}}(m)\right)^{\top} \vec{a}(m+l) d_{\bar{\mu}}(l-k),
$$

and $\varphi_{\bar{\mu}, N}=\left(\varphi_{\bar{\mu}}(0), \varphi_{\bar{\mu}}(1), \ldots, \varphi_{\bar{\mu}}(N)\right) ; \mathbf{A}_{N}$ is a linear operator determined by the coefficients $\vec{a}(k), k=$ $0,1, \ldots, N$, as follows: $\left(\mathbf{A}_{N}\right)(k, j)=\vec{a}(k+j), 0 \leq k+j \leq N,\left(\mathbf{A}_{N}\right)(k, j)=0, k+j>N, 0 \leq k, j \leq N ; \widetilde{D}_{N}^{\bar{\mu}}$ is a matrix of the dimension $(N+1) \times(N+1)$ determined by the $T \times T$ entries $\widetilde{D}_{N}^{\bar{\mu}}(k, j)=\operatorname{diag}_{T}\left(d_{\bar{\mu}}(j-k)\right)$ if $0 \leq k \leq j \leq N$ and $\widetilde{D}_{N}^{\bar{\mu}}(k, j)=\operatorname{diag}_{T}(0)$ if $0 \leq j<k \leq N$.

The value of the mean square error is calculated by the formula

$$
\begin{aligned}
\Delta\left(f ; \widehat{A}_{N} \vec{\xi}\right) & =\frac{1}{2 \pi} \int_{-\pi}^{\pi}\left(\sum_{k=0}^{N}\left(\widetilde{D}_{N}^{\bar{\mu}} \mathbf{A}_{N} \varphi_{\bar{\mu}, N}\right)_{k} e^{i \lambda k}\right)^{\top}\left(\overline{\sum_{k=0}^{N}\left(\widetilde{D}_{N}^{\bar{\mu}} \mathbf{A}_{N} \varphi_{\bar{\mu}, N}\right)_{k} e^{i \lambda k}}\right) d \lambda \\
& =\frac{1}{2 \pi} \int_{-\pi}^{\pi}\left\|\vec{r}_{\bar{\mu}, N}\left(e^{i \lambda}\right)\right\|^{2} d \lambda=\left\|\widetilde{D}_{N}^{\bar{\mu}} \mathbf{A}_{N} \varphi_{\bar{\mu}, N}\right\|^{2} .
\end{aligned}
$$

Thus, the following theorem holds true.

Theorem 3.2

Let $\{\vec{\xi}(m), m \in \mathbb{Z}\}$ be a stochastic sequence which determine a stationary stochastic GM increment sequence $\chi_{\bar{\mu}, \bar{s}}^{(n)}(\vec{\xi}(m))$ with the spectral density matrix $f(\lambda)$ which satisfies the minimality condition (23). The optimal linear 
estimate $\widehat{A}_{N} \vec{\xi}$ of the functional $A_{N} \vec{\xi}$ based on observations of the sequence $\vec{\xi}(m)$ at points $m=-1,-2, \ldots$ is calculated by formula (41). The spectral characteristic $\vec{h}_{\bar{\mu}, N}(\lambda)=\left\{h_{\mu, N, p}(\lambda)\right\}_{p=1}^{T}$ and the value of the mean square error $\Delta\left(f ; \widehat{A}_{N} \vec{\xi}\right)$ are calculated by formulas (42) and (43) respectively. In the case where the spectral density $f(\lambda)$ admits the canonical factorization (38) the spectral characteristic $\vec{h}_{\bar{\mu}, N}(\lambda)$ and the value of the mean square error of the estimate $\widehat{A}_{N} \vec{\xi}$ can be calculated by formulas (44) and (45) respectively.

For the problem of the mean square optimal estimate of the unobserved value $A_{N, p} \vec{\xi}=\xi_{p}(N)=\vec{\xi}(N) \boldsymbol{\delta}_{p}$, $p=1,2, \ldots, T, N \geq 0$ of the stochastic sequence $\vec{\xi}(m)$ with GM stationary increments based on its observations at points $m=-1,-2, \ldots$ we have the following corollary from Theorem 3.2.

\section{Corollary 3.2}

The optimal linear estimate $\widehat{\xi}_{p}(N)$ of the value $\xi_{p}(N), p=1,2, \ldots, T, N \geq 0$, of the stochastic sequence with GM stationary increments from observations of the sequence $\vec{\xi}(m)$ at points $m=-1,-2, \ldots$ is calculated by formula

$$
\widehat{\xi}_{p}(N)=\int_{-\pi}^{\pi}\left(\vec{h}_{\bar{\mu}, N, p}(\lambda)\right)^{\top} d \vec{Z}_{\xi^{(d)}}(\lambda)-\sum_{k=-n(\gamma)}^{-1}\left(\vec{v}_{N, p}(k)\right)^{\top} \vec{\xi}(k) .
$$

The spectral characteristic $\vec{h}_{\bar{\mu}, N, p}(\lambda)$ of the estimate is calculated by the formula

$$
\left(\vec{h}_{\bar{\mu}, N, p}(\lambda)\right)^{\top}=\frac{\chi_{\bar{\mu}}^{(d)}\left(e^{-i \lambda}\right)}{\beta^{(d)}(i \lambda)}\left(\boldsymbol{\delta}_{p} \sum_{k=0}^{N} d_{\bar{\mu}}(N-k) e^{i \lambda k}\right)^{\top}-\frac{\overline{\beta^{(d)}(i \lambda)}}{\chi_{\bar{\mu}}^{(d)}\left(e^{i \lambda}\right)}\left(\sum_{k=0}^{\infty}\left(\mathbf{F}_{\bar{\mu}}^{-1} \mathbf{d}_{\bar{\mu}, N, p)}\right)_{k} e^{i \lambda k}\right)^{\top} f^{-1}(\lambda) .
$$

where $\mathbf{d}_{\bar{\mu}, N, p}=\left(d_{\bar{\mu}}(N) \boldsymbol{\delta}_{p}^{\top}, d_{\bar{\mu}}(N-1) \boldsymbol{\delta}_{p}^{\top}, \ldots, d_{\bar{\mu}}(0) \boldsymbol{\delta}_{p}^{\top}, 0, \ldots\right)^{\top}$. The value of the mean square error of the estimate $\widehat{\xi}_{p}(N)$ is calculated by the formula

$$
\begin{aligned}
& \Delta\left(f ; \widehat{\xi}_{p}(N)\right)=\Delta\left(f ; \chi_{\bar{\mu}, \bar{s}}^{(n)}\left(\widehat{\xi}_{p}(m)\right)\right)=\mathrm{E}\left|\chi_{\bar{\mu}, \bar{s}}^{(n)}\left(\xi_{p}(m)\right)-\chi_{\bar{\mu}, \bar{s}}^{(n)}\left(\widehat{\xi}_{p}(m)\right)\right|^{2} \\
& =\frac{1}{2 \pi} \int_{-\pi}^{\pi} \frac{\overline{\beta^{(d)}(i \lambda)}}{\chi_{\bar{\mu}}^{(d)}\left(e^{i \lambda}\right)}\left(\sum_{k=0}^{\infty}\left(\mathbf{F}_{\bar{\mu}}^{-1} \mathbf{d}_{\bar{\mu}, N, p}\right)_{k} e^{i \lambda k}\right)^{\top} f(\lambda)\left(\overline{\left.\sum_{k=0}^{\infty}\left(\mathbf{F}_{\bar{\mu}}^{-1} \mathbf{d}_{\bar{\mu}, N, p}\right)_{k} e^{i \lambda k}\right)} \frac{\beta^{(d)}(i \lambda)}{\chi_{\bar{\mu}}^{(d)}\left(e^{-i \lambda}\right)} d \lambda\right. \\
& =\left\langle\mathbf{d}_{\bar{\mu}, N, p}, \mathbf{F}_{\bar{\mu}}^{-1} \mathbf{d}_{\bar{\mu}, N, p}\right\rangle \text {. }
\end{aligned}
$$

In the case where the spectral density $f(\lambda)$ admits the canonical factorization (38), and the condition $\min _{i=\overline{1, r}} \mu_{i} s_{i}>N$ is satisfied, the spectral characteristic and the value of the mean square error of the estimate $\widehat{\xi}_{p}(N)$ can be calculated by the formulas

$$
\vec{h}_{\bar{\mu}, N, p}(\lambda)=\frac{\chi_{\bar{\mu}}^{(d)}\left(e^{-i \lambda}\right)}{\beta^{(d)}(i \lambda)} e^{i N \lambda}\left[\boldsymbol{\delta}_{p}-\left(\Psi_{\bar{\mu}}\left(e^{-i \lambda}\right)\right)^{\top}\left(\sum_{k=0}^{N} \varphi_{\bar{\mu}}(k) e^{-i \lambda k}\right)^{\top} \boldsymbol{\delta}_{p}\right]
$$

and

$$
\Delta\left(f ; \widehat{\xi}_{p}(N)\right)=\frac{1}{2 \pi} \int_{-\pi}^{\pi}\left[\left(\boldsymbol{\delta}_{p}\right)^{\top} \sum_{k=0}^{N} \varphi_{\bar{\mu}}(k) e^{-i \lambda k}\right]\left[\left(\boldsymbol{\delta}_{p}\right)^{\top} \sum_{k=0}^{N} \varphi_{\bar{\mu}}(k) e^{-i \lambda k}\right]^{*} d \lambda=\sum_{k=0}^{N} \sum_{j=1}^{q}\left|\varphi_{\mu, p, j}(k)\right|^{2} .
$$

Remark 3.1

Since for all $\bar{d} \geq \overline{1}$ and $\bar{\mu} \geq \overline{1}$ the condition

$$
\int_{-\pi}^{\pi}\left|\ln \frac{\left|\chi_{\bar{\mu}}^{(d)}\left(e^{-i \lambda}\right)\right|^{2}}{\left|\beta^{(d)}(i \lambda)\right|^{2}}\right| d \lambda<\infty
$$


holds true, there exists a function

$$
w_{\bar{\mu}}(z)=\sum_{k=0}^{\infty} w_{\bar{\mu}}(k) z^{k}, \quad \sum_{k=0}^{\infty}\left|w_{\bar{\mu}}(k)\right|^{2}<\infty
$$

such that [18]

$$
\left|w_{\bar{\mu}}\left(e^{-i \lambda}\right)\right|^{2}=\frac{\left|\chi_{\bar{\mu}}^{(d)}\left(e^{-i \lambda}\right)\right|^{2}}{\left|\beta^{(d)}(i \lambda)\right|^{2}}
$$

which can be calculated by the formula

$$
w_{\bar{\mu}}(z)=\exp \left\{\frac{1}{4 \pi} \int_{-\pi}^{\pi} \frac{e^{i \lambda}+z}{e^{i \lambda}-z} \ln \frac{\left|\chi_{\bar{\mu}}^{(d)}\left(e^{-i \lambda}\right)\right|^{2}}{\left|\beta^{(d)}(i \lambda)\right|^{2}} d \lambda\right\} .
$$

For this reason the following relation holds true:

$$
\Phi_{\bar{\mu}}\left(e^{-i \lambda}\right)=w_{\bar{\mu}}\left(e^{-i \lambda}\right) \Phi\left(e^{-i \lambda}\right) .
$$

which implies

$$
\varphi_{\bar{\mu}}(k)=\sum_{m=0}^{k} w_{\bar{\mu}}(k-m) \varphi(m), \quad k=0,1, \ldots
$$

that is

$$
\varphi_{\mu, i j}(k)=\sum_{m=0}^{k} w_{\bar{\mu}}(k-m) \varphi_{i j}(m), \quad i=1, \ldots, T ; j=1, \ldots, q ; k=0,1, \ldots
$$

This relation can be represented in the form

$$
\varphi_{\bar{\mu}}=\mathbf{W}^{\bar{\mu}} \boldsymbol{\varphi}
$$

where $\varphi_{\bar{\mu}}=\left(\varphi_{\bar{\mu}}(0), \varphi_{\bar{\mu}}(1), \varphi_{\bar{\mu}}(2), \ldots\right)^{\top}$ and $\varphi=(\varphi(0), \varphi(1), \varphi(2), \ldots)^{\top}$ are vectors composed from matrices $\varphi_{\bar{\mu}}(k)=\left\{\varphi_{\mu, i j}(k)\right\}_{i=\overline{1, T}}^{j=\overline{1, q}}, k=0,1,2, \ldots$, and $\varphi(k)=\left\{\varphi_{i j}(k)\right\}_{i=\overline{1, T}}^{j=\overline{1, q}}, k=0,1,2, \ldots$, and where $\mathbf{W}^{\bar{\mu}}$ is a linear operator with the entries $\left(\mathbf{W}^{\bar{\mu}}\right)_{j, k}=w_{\bar{\mu}}(j-k)$ if $0 \leq k \leq j$, and $\left(\mathbf{W}^{\bar{\mu}}\right)_{j, k}=0$ if $0 \leq j<k$.

\subsection{Forecasting of periodically stationary GM increment}

Consider the problem of mean square optimal linear estimation of the functionals

$$
A \zeta=\sum_{k=0}^{\infty} a^{(\zeta)}(k) \zeta(k), \quad A_{M} \zeta=\sum_{k=0}^{N} a^{(\zeta)}(k) \zeta(k)
$$

which depend on unobserved values of the stochastic sequence $\zeta(m)$ with periodically stationary increments. Estimates are based on observations of the sequence $\zeta(m)$ at points $m=-1,-2, \ldots$.

The functional $A \zeta$ can be represented in the form

$$
\begin{aligned}
A \zeta & =\sum_{k=0}^{\infty} a^{(\zeta)}(k) \zeta(k)=\sum_{m=0}^{\infty} \sum_{p=1}^{T} a^{(\zeta)}(m T+p-1) \zeta(m T+p-1) \\
& =\sum_{m=0}^{\infty} \sum_{p=1}^{T} a_{p}(m) \xi_{p}(m)=\sum_{m=0}^{\infty}(\vec{a}(m))^{\top} \vec{\xi}(m)=A \vec{\xi}
\end{aligned}
$$

where

$$
\vec{\xi}(m)=\left(\xi_{1}(m), \xi_{2}(m), \ldots, \xi_{T}(m)\right)^{\top}, \xi_{p}(m)=\zeta(m T+p-1) ; p=1,2, \ldots, T ;
$$




$$
\vec{a}(m)=\left(a_{1}(m), a_{2}(m), \ldots, a_{T}(m)\right)^{\top}, a_{p}(m)=a^{(\zeta)}(m T+p-1) ; p=1,2, \ldots, T .
$$

Making use of the introduced notations and statements of Theorem 3.1 we can claim that the following theorem holds true.

\section{Theorem 3.3}

Let a stochastic sequence $\zeta(k)$ with periodically stationary increments generate by formula (55) a vector-valued stochastic sequence $\vec{\xi}(m)$ which determine a stationary stochastic GM increment sequence $\chi_{\bar{\mu}, \bar{s}}^{(n)}(\vec{\xi}(m))$ with the spectral density matrix $f(\lambda)=\left\{f_{i j}(\lambda)\right\}_{i, j=1}^{T}$ that satisfy the minimality condition (23). Let the coefficients $\vec{a}(k), k \geqslant 0$ determined by formula (56) satisfy conditions (21) - (22).

Then the optimal linear estimate $\widehat{A} \zeta$ of the functional $A \zeta$ based on observations of the sequence $\zeta(m)$ at points $m=-1,-2, \ldots$ is calculated by formula (28). The spectral characteristic $\vec{h}_{\bar{\mu}}(\lambda)=\left\{h_{p}(\lambda)\right\}_{p=1}^{T}$ and the value of the mean square error $\Delta(f ; \widehat{A} \zeta)$ of the estimate $\widehat{A} \zeta$ are calculated by formulas (36) and (37) respectively.

In the case where the spectral density matrix $f(\lambda)$ admits the canonical factorization (38), the spectral characteristic and the value of the mean square error of the estimate $\widehat{A} \xi$ can be calculated by formulas (39) and (40) respectively.

The functional $A_{M} \zeta$ can be represented in the form

$$
\begin{aligned}
A_{M} \zeta & =\sum_{k=0}^{M} a^{(\zeta)}(k) \zeta(k)=\sum_{m=0}^{N} \sum_{p=1}^{T} a^{(\zeta)}(m T+p-1) \zeta(m T+p-1) \\
& =\sum_{m=0}^{N} \sum_{p=1}^{T} a_{p}(m) \xi_{p}(m)=\sum_{m=0}^{N}(\vec{a}(m))^{\top} \vec{\xi}(m)=A_{N} \vec{\xi},
\end{aligned}
$$

where $N=\left[\frac{M}{T}\right]$, the sequence $\vec{\xi}(m)$ is determined by formula (55),

$$
\begin{aligned}
(\vec{a}(m))^{\top} & =\left(a_{1}(m), a_{2}(m), \ldots, a_{T}(m)\right)^{\top}, \\
a_{p}(m) & =a^{\zeta}(m T+p-1) ; 0 \leq m \leq N ; 1 \leq p \leq T ; m T+p-1 \leq M ; \\
a_{p}(N) & =0 ; \quad M+1 \leq N T+p-1 \leq(N+1) T-1 ; 1 \leq p \leq T .
\end{aligned}
$$

Making use of the introduced notations and statements of Theorem 3.2 we can claim that the following theorem holds true.

\section{Theorem 3.4}

Let a stochastic sequence $\zeta(k)$ with periodically stationary GM increments generate by formula (55) a vectorvalued stochastic sequence $\vec{\xi}(m)$ which determine a stationary GM increment sequence $\chi_{\bar{\mu}, \bar{s}}^{(n)}(\vec{\xi}(m))$ with the spectral density matrix $f(\lambda)=\left\{f_{i j}(\lambda)\right\}_{i, j=1}^{T}$ that satisfy the minimality condition (23). Let coefficients $\vec{a}(k), k \geqslant$ 0 be determined by formula (57). The optimal linear estimate $\widehat{A}_{M} \zeta$ of the functional $A_{M} \zeta=A_{N} \vec{\xi}$ based on observations of the sequence $\zeta(m)$ at points $m=-1,-2, \ldots$ is calculated by formula (41). The spectral characteristic $\vec{h}_{\bar{\mu}, N}(\lambda)=\left\{h_{\bar{\mu}, N, p}(\lambda)\right\}_{p=1}^{T}$ and the value of the mean square error $\Delta\left(f ; \widehat{A}_{M} \zeta\right)$ are calculated by formulas (42) and (43) respectively. In the case where the spectral density matrix $f(\lambda)$ admits the canonical factorization (38), then the spectral characteristic $\vec{h}_{\bar{\mu}, N}(\lambda)$ and the value of the mean square error of the estimate $\widehat{A}_{M} \zeta$ can be calculated by formulas (44) and (45) respectively.

As a corollary from Theorem 3.4, one can obtain the mean square optimal estimate of the unobserved value $\zeta(M), M \geq 0$ of a stochastic sequence $\zeta(m)$ with periodically stationary GM increments based on observations of the sequence $\zeta(m)$ at points $m=-1,-2, \ldots$ Making use of the notations $\zeta(M)=\xi_{p}(N)=(\vec{\xi}(N))^{\top} \boldsymbol{\delta}_{p}, N=\left[\frac{M}{T}\right]$, $p=M+1-N T$, and the obtained results we can conclude that the following corollary holds true.

\section{Corollary 3.3}

Let a stochastic sequence $\zeta(m)$ with periodically stationary GM increments generate by formula (55) a vectorvalued stochastic sequence $\vec{\xi}(m)$ which determine a stationary GM increment sequence $\chi_{\bar{\mu}, \bar{s}}^{(n)}(\vec{\xi}(m))$ with the 
spectral density matrix $f(\lambda)=\left\{f_{i j}(\lambda)\right\}_{i, j=1}^{T}$ that satisfy the minimality condition (23). The optimal linear estimate $\widehat{\zeta}(M)$ of the unobserved value $\zeta(M), M \geq 0$, based on observations of the sequence $\zeta(m)$ at points $m=-1,-2, \ldots$ is calculated by formula (46). The spectral characteristic $\vec{h}_{\bar{\mu}, N, p}(\lambda)$ of the estimate is calculated by the formula (47). The value of the mean square error of the estimate $\widehat{\zeta}(M)$ is calculated by the formula (48). In the case where the spectral density $f(\lambda)$ admits the canonical factorization (38), then the spectral characteristic and the value of the mean square error of the estimate $\widehat{\zeta}(M)$ can be calculated by the formulas (49), (50).

\section{Forecasting of GM fractional increments}

In the previous section, we solved the forecasting problem for the increment sequence $\chi_{\bar{\mu}, \bar{s}}^{(d)}(\vec{\xi}(m))$ of positive integer orders $\left(d_{1}, \ldots, d_{r}\right)$. Here we consider the forecasting problem in the case of fractional increment orders $d_{i}$.

Within the section, we consider the step $\bar{\mu}=(1,1, \ldots, 1)$ and represent the increment operator $\chi_{\bar{s}}^{(d)}(B)$ in the form

$$
\chi_{\bar{s}}^{(R+D)}(B)=(1-B)^{R_{0}+D_{0}} \prod_{j=1}^{r}\left(1-B^{s_{j}}\right)^{R_{j}+D_{j}},
$$

where $(1-B)^{R_{0}+D_{0}}$ is the integrating component, $R_{j}, j=0,1, \ldots, r$, are non-negative integer numbers, $1<$ $s_{1}<\ldots<s_{r}$. The goal is to find representations $d_{j}=R_{j}+D_{j}, j=0,1, \ldots, r$, of the increment orders under some conditions on the fractional parts $D_{j}$, such that the increment sequence

$$
\vec{y}(m):=(1-B)^{R_{0}} \prod_{j=1}^{r}\left(1-B^{s_{j}}\right)^{R_{i}} \vec{\xi}(m)
$$

to be a stationary fractionally integrated seasonal stochastic sequence. For example, in case of single increment pattern $\left(1-B^{s^{*}}\right)^{R^{*}+D^{*}}$ this condition is $\left|D^{*}\right|<1 / 2$.

We will call a sequence $\chi_{\bar{s}}^{(R+D)}(\vec{\xi}(m))$ fractional multiple $(F M)$ increment sequence.

\section{Lemma 4.1}

The increment operator $\chi_{\bar{s}}^{(D)}(B):=(1-B)^{D_{0}} \prod_{j=1}^{r}\left(1-B^{s_{j}}\right)^{D_{j}}$ admits a representation

$$
\chi_{\bar{s}}^{(D)}(B)=\prod_{j=0}^{r} \prod_{k_{j}=0}^{\left[s_{j} / 2\right]}\left(1-2 \cos \nu_{k_{j}} B+B^{2}\right)^{D_{k_{j}}}=(1-B)^{D_{0}+D_{1}+\ldots+D_{r}} \prod_{j=1}^{r} \prod_{k_{j}=1}^{\left[s_{j} / 2\right]}\left(1-2 \cos \nu_{k_{j}} B+B^{2}\right)^{D_{k_{j}}},
$$

where $s_{0}=1, \nu_{k_{j}}=2 \pi k_{j} / s_{j}, k_{j}=0,1, \ldots,\left[s_{j} / 2\right], D_{k_{j}}=D_{0} / 2$ for $k_{j}=0, D_{k_{j}}=D_{j}$ for $k_{j}=1,2, \ldots,\left[s_{j} / 2\right]-$ $1, D_{\left[s_{j} / 2\right]}=D_{j}$ for odd $s_{j}$ and $D_{\left[s_{j} / 2\right]}=D_{j} / 2$ for even $s_{j}$,

Note that Lemma 4.1 follows from the representation

$$
\left(1-B^{s_{j}}\right)^{D_{r}}=\prod_{k_{j}=0}^{\left[s_{j} / 2\right]}\left(z_{k_{j}}-B\right)^{D_{k_{j}}}\left(z_{-k_{j}}-B\right)^{D_{k_{j}}},
$$

where $z_{k_{j}}=\exp \left(i \nu_{k_{j}}\right), k_{j}=0,1, \ldots, s_{j}-1$, are solutions of the equation $1-B^{s_{j}}=0$.

Lemma 4.1 implies the following statement.

\section{Lemma 4.2}

Define the sets $\mathcal{M}_{j}=\left\{\nu_{k_{j}}=2 \pi k_{j} / s_{j}: k_{j}=0,1, \ldots,\left[s_{j} / 2\right]\right\}, j=0,1, \ldots, r$, and the set $\mathcal{M}=\bigcup_{j=0}^{r} \mathcal{M}_{j}$. Then

$$
\begin{aligned}
\chi_{\bar{s}}^{(D)}(B) & =\prod_{\nu \in \mathcal{M}}\left(1-2 \cos \nu B+B^{2}\right)^{\widetilde{D}_{\nu}} \\
& =(1-B)^{D_{0}+D_{1}+\ldots+D_{r}}(1+B)^{D_{\pi}} \prod_{\nu \in \mathcal{M} \backslash\{0, \pi\}}\left(1-2 \cos \nu B+B^{2}\right)^{D_{\nu}},
\end{aligned}
$$


where $D_{\nu}=\sum_{j=0}^{r} D_{j} \mathbb{I}\left\{\nu \in \mathcal{M}_{j}\right\}, \widetilde{D}_{\nu}=D_{\nu}$ for $\nu \in \mathcal{M} \backslash\{0, \pi\}, \widetilde{D}_{\nu}=D_{\nu} / 2$ for $\nu=0$ and $\nu=\pi$.

Lemma 4.2 shows that a multiple seasonal increment process can be represented as a $k$-factor Gegenbauer process

$$
\prod_{i=1}^{k}\left(1-2 u_{i} B+B^{2}\right)^{d_{i}} x(m)=\xi(m)
$$

In the case where $\xi(m)$ is an $A R M A(p, q)$ sequence, the model $x(m)$ defined by (59) is called $k$-factor $\operatorname{GARMA}\left(p, d_{i}, u_{i}, q\right)$ sequence. It is stationary and invertible if $\left|d_{i}\right|<1 / 2$ for $\left|u_{i}\right|<1$ and $\left|d_{i}\right|<1 / 4$ for $\left|u_{i}\right|=1$. If additionally $d_{i}>0$, then the model exhibits a long memory behavior [50]. The function $\left(1-2 u_{i} B+B^{2}\right)^{-d_{i}}$ is a generating function of the Gegenbauer polynomial:

$$
\left(1-2 u B+B^{2}\right)^{-d}=\sum_{n=0}^{\infty} C_{n}^{(d)}(u) B^{n},
$$

where

$$
C_{n}^{(d)}(u)=\sum_{k=0}^{[n / 2]} \frac{(-1)^{k}(2 u)^{n-2 k} \Gamma(d-k+n)}{k !(n-2 k) ! \Gamma(d)} .
$$

Thus, denoting $k^{*}=|\mathcal{M}|$, we obtain

$$
\left(\chi_{\bar{s}}^{(D)}(B)\right)^{-1}=\prod_{\nu \in \mathcal{M}}\left(1-2 \cos \nu B+B^{2}\right)^{-\widetilde{D}_{\nu}}=\sum_{m=0}^{\infty} G_{k^{*}}^{+}(m) B^{m}=\left(\sum_{m=0}^{\infty} G_{k^{*}}^{-}(m) B^{m}\right)^{-1},
$$

where

$$
\begin{aligned}
G_{k^{*}}^{+}(m) & =\sum_{0 \leq n_{1}, \ldots, n_{k^{*}} \leq m, n_{1}+\ldots+n_{k^{*}}=m} \prod_{\nu \in \mathcal{M}} C_{n_{\nu}}^{\left(\widetilde{D}_{\nu}\right)}(\cos \nu), \\
G_{k^{*}}^{-}(m) & \sum_{0 \leq n_{1}, \ldots, n_{k^{*}} \leq m, n_{1}+\ldots+n_{k^{*}}=m} \prod_{\nu \in \mathcal{M}} C_{n_{\nu}}^{\left(-\widetilde{D}_{\nu}\right)}(\cos \nu) .
\end{aligned}
$$

The derived representations of the increment operator $\chi_{\bar{s}}^{(D)}(B)$ imply the following theorem.

Theorem 4.1

Assume that for a stochastic vector sequence $\vec{\xi}(m)$ and fractional differencing orders $d_{j}=R_{j}+D_{j}, j=$ $0,1, \ldots, r$, the FM increment sequence $\chi_{\overline{1}, \bar{s}}^{(R+D)}(\vec{\xi}(m))$ generated by increment operator (58) is a stationary sequence with a bounded from zero and infinity spectral density $\widetilde{f}_{\overline{1}}(\lambda)$. Then for the non-negative integer numbers $R_{j}, j=0,1, \ldots, r$, the GM increment sequence $\chi_{\overline{1}, \bar{s}}^{(R)}(\vec{\xi}(m))$ is stationary if $-1 / 2<D_{\nu}<1 / 2$ for all $\nu \in \mathcal{M}$, where $D_{\nu}$ are defined by real numbers $D_{j}, j=0,1, \ldots, r$, in Lemma 4.2, and it is long memory if $0<D_{\nu}<1 / 2$ for at least one $\nu \in \mathcal{M}$, and invertible if $-1 / 2<D_{\nu}<0$. The spectral density $f(\lambda)$ of the stationary GM increment sequence $\chi_{\overline{1}, \bar{s}}^{(R)}(\vec{\xi}(m))$ admits a representation

$$
f(\lambda)=\left|\beta^{(R)}(i \lambda)\right|^{2}\left|\chi_{\overline{1}}^{(R)}\left(e^{-i \lambda}\right)\right|^{-2}\left|\chi_{\overline{1}}^{(D)}\left(e^{-i \lambda}\right)\right|^{-2} \widetilde{f}_{\overline{1}}(\lambda)=:\left|\chi_{\overline{1}}^{(D)}\left(e^{-i \lambda}\right)\right|^{-2} \widetilde{f}(\lambda),
$$

where

$$
\left|\chi_{\overline{1}}^{(D)}\left(e^{-i \lambda}\right)\right|^{-2}=\left|\sum_{m=0}^{\infty} G_{k^{*}}^{+}(m) e^{-i \lambda m}\right|^{2}=\left|\sum_{m=0}^{\infty} G_{k^{*}}^{-}(m) e^{-i \lambda m}\right|^{-2}=\prod_{\nu \in \mathcal{M}}\left|\left(e^{-i \nu}-e^{i \lambda}\right)\left(e^{i \nu}-e^{i \lambda}\right)\right|^{-2 \widetilde{D}_{\nu}},
$$

coefficients $G_{k^{*}}^{+}(m), G_{k^{*}}^{-}(m)$ are defined by (60), (61). 
The spectral density $f(\lambda)$ and the structural function $D_{\bar{s}}^{(R)}(m, \overline{1}, \overline{1})$ of a stationary GM increment sequence $\chi_{\overline{1}, \bar{s}}^{(R)}(\vec{\xi}(m))$ exhibit the following behavior in the case of constant matrices $C$ and $K$ :

- $\left|\beta^{(R)}(i \lambda)\right|^{-2}\left|\chi_{\overline{1}}^{(R)}\left(e^{-i \lambda}\right)\right|^{2} f(\lambda) \sim C|\nu-\lambda|^{-2 \widetilde{D}_{\nu}}$ as $\lambda \rightarrow \nu, \nu \in \mathcal{M}$, thus, the minimality condition (23) is satisfied (for properties of eigenvalues of generalized fractional process, we refer to Palma and Bondon [41])

- $D_{\bar{s}}^{(R)}(m, \overline{1}, \overline{1}) \sim K \sum_{\nu \in \mathcal{M}: \widetilde{D}_{\nu}>0}|m|^{2 \widetilde{D}_{\nu}-1} \cos (m \nu)$, as $m \rightarrow \infty$ (see Giraitis and Leipus [12]).

\section{Example 4.1}

1. Consider an increment operator $(1-B)^{R_{0}+D_{0}}\left(1-B^{2}\right)^{R_{1}+D_{1}}$ which represents a fractional integrated component and a fractional seasonal components. In this case $\mathcal{M}_{0}=\{0\}, \mathcal{M}_{1}=\{0, \pi\}, \mathcal{M}=\{0, \pi\}$. The Gegenbauer representation of the increment is $(1-B)^{D_{0}+D_{1}}(1+B)^{D_{1}}$. Stationarity conditions are the following: $|D|=\left|D_{0}+D_{1}\right|<1 / 2,\left|D_{\pi}\right|=\left|D_{1}\right|<1 / 2$.

2. Consider an increment operator $\left(1-B^{2}\right)^{R_{1}+D_{1}}\left(1-B^{3}\right)^{R_{2}+D_{2}}$ which represents two fractional seasonal components. In this case $\mathcal{M}_{0}=\{0, \pi\}, \mathcal{M}_{1}=\{0,2 \pi / 3\}, \mathcal{M}=\{0,2 \pi / 3, \pi\}$. The Gegenbauer representation of the increment is $(1-B)^{D_{1}+D_{2}}\left(1-2 \cos (2 \pi / 3) B+B^{2}\right)^{D_{2}}(1+B)^{D_{1}}$. Stationarity conditions are the following: $|D|=\left|D_{1}+D_{2}\right|<1 / 2,\left|D_{2 \pi / 3}\right|=\left|D_{2}\right|<1 / 2,\left|D_{\pi}\right|=\left|D_{1}\right|<1 / 2$.

3. Consider an increment operator $\left(1-B^{2}\right)^{R_{1}+D_{1}}\left(1-B^{4}\right)^{R_{2}+D_{2}}$. In this case $\mathcal{M}_{0}=\{0, \pi\}, \mathcal{M}_{1}=\{0, \pi / 2, \pi\}$, $\mathcal{M}=\{0, \pi / 2, \pi\}$. The Gegenbauer representation of the increment is $(1-B)^{D_{1}+D_{2}}\left(1+B^{2}\right)^{D_{2}}(1+B)^{D_{1}+D_{2}}$. Stationarity conditions are the following: $|D|=\left|D_{\pi}\right|=\left|D_{1}+D_{2}\right|<1 / 2,\left|D_{\pi / 2}\right|=\left|D_{2}\right|<1 / 2$.

In the following remarks we provide some additional details with the help of which we can use theorems proposed in the previous section in finding solution of the forecasting problem for stochastic sequences with periodically stationary (periodically correlated) FM increments.

\section{Remark 4.1}

Theorem 4.1 implies that the Fourier coefficients (33) of the function

$$
\left|\beta^{(R)}(i \lambda)\right|^{2}\left|\chi_{\overline{1}}^{(R)}\left(e^{-i \lambda}\right)\right|^{-2} f^{-1}(\lambda)
$$

are calculated by the formula

$$
F^{\overline{1}}(k, j)=\frac{1}{2 \pi} \int_{-\pi}^{\pi} e^{i \lambda(j-k)}\left|\chi_{\overline{1}}^{(D)}\left(e^{-i \lambda}\right)\right|^{2} \widetilde{f}_{\overline{1}}^{-1}(\lambda) d \lambda, \quad k, j \geq 0 .
$$

\section{Remark 4.2}

Assume that the spectral density $\widetilde{f}_{\overline{1}}(\lambda)$ admits a factorization

$$
\widetilde{f}_{\overline{1}}(\lambda)=\left|\widetilde{\Phi}_{\overline{1}}\left(e^{-i \lambda}\right)\right|^{2}=\left|\sum_{k=0}^{\infty} \widetilde{\varphi}_{\overline{1}}(k)\left(e^{-i \lambda}\right)\right|^{2},
$$

where $\widetilde{\varphi}_{\overline{1}}(k)=\left\{\varphi_{\overline{1}, i j}(k)\right\}_{i=\overline{1, T}}^{j=\overline{1, q}}, k=0,1,2, \ldots$ Then coefficients $\left\{\varphi_{\overline{1}, i j}\right\}_{i=1, T}^{j=\overline{1, q}}, k=0,1,2, \ldots$ from factorization (38) are calculated by the formula

$$
\varphi_{\overline{1}, i j}(k)=\sum_{m=0}^{k} G_{k^{*}}^{+}(k-m) \widetilde{\varphi}_{\overline{1}, i j}(m)=\left(G_{k^{*}}^{+} * \widetilde{\varphi}_{\overline{1}, i j}\right)(k) .
$$

Remark 4.3

Define a matrix-valued function $\widetilde{\Psi}_{\overline{1}}\left(e^{-i \lambda}\right)=\left\{\widetilde{\Psi}_{\overline{1}, i j}\left(e^{-i \lambda}\right)\right\}_{i=\overline{1, q}}^{j=\overline{1, T}}$ by the equation $\widetilde{\Psi}_{\overline{1}}\left(e^{-i \lambda}\right) \widetilde{\Phi}_{\overline{1}}\left(e^{-i \lambda}\right)=E_{q}$, where $E_{q}$ is the identity $q \times q$ matrix. Then $\Psi_{\overline{1}}\left(e^{-i \lambda}\right)=\chi_{\overline{1}}^{(D)}\left(e^{-i \lambda}\right) \widetilde{\Psi}_{\overline{1}}\left(e^{-i \lambda}\right)$. 


\section{Examples of forecasting for some special models}

\section{Example 5.1}

Basawa et al. [5] consider a so-called first-order seasonal periodic autoregressive process (SPAR $(1,1))$ defined by the difference equation

$$
X_{n T+\nu}=\phi(\nu) X_{m T+\nu-1}+\alpha(\nu) X_{(m-1) T+\nu}-\phi(\nu) \alpha(\nu) X_{(m-1) T+\nu-1}+\varepsilon_{m T+\nu},
$$

where $\varepsilon_{m T+\nu}$ is an uncorrelated periodic white noise process with $\mathrm{E}\left(\varepsilon_{m T+\nu}\right)=0$ and $\operatorname{Var}\left(\varepsilon_{m T+\nu}\right)=\sigma^{2}(\nu)$, $1 \leq \nu \leq T$ (we follow notations from [5]). Depending on coefficients $\phi(\nu), \alpha(\nu)$ model (62) has the following properties:

- if $\phi(\nu) \equiv \phi, \alpha(\nu) \equiv \alpha, \sigma^{2}(\nu) \equiv \sigma^{2}$ for $1 \leq \nu \leq T$, then model (62) reduces to Box-Jenkins SAR(1,1) model,

- if $\alpha(\nu) \equiv 0$ for $1 \leq \nu \leq T$, then model (62) reduces to PAR(1) model,

- if $\prod_{\nu=1}^{T}|\phi(\nu)|<1$ and $|\alpha(\nu)|<1$ for all $\nu$, then model (62) admits a causal and stationary $T$-dimensional VAR representation

$$
\mathbf{\Phi}_{0} \mathbf{X}_{m}=\boldsymbol{\Phi}_{1} \mathbf{X}_{m-1}+\boldsymbol{\Phi}_{2} \mathbf{X}_{m-2}+\boldsymbol{\varepsilon}_{n}
$$

where

$$
\mathbf{X}_{m}=\left(X_{m T+1}, X_{m T+2}, \ldots, X_{m T+T}\right)^{\top}, \quad \varepsilon_{m}=\left(\varepsilon_{m T+1}, \varepsilon_{m T+2}, \ldots, \varepsilon_{m T+T}\right)^{\top} .
$$

Here we consider another case where $\prod_{\nu=1}^{T}|\phi(\nu)|<1$ and $\alpha(\nu) \equiv 1$ for all $\nu$. Without loss of generality assume that $\sigma^{2}(\nu) \equiv 1$ for all $\nu$. Then, taking into account $B^{T} X_{m T+\nu}=X_{(m-1) T+\nu}$, model (62) reduces to integrated PAR, or PARIMA, model

$$
\left(1-B^{T}\right)\left(X_{m T+\nu}-\phi(\nu) X_{m T+\nu-1}\right)=\varepsilon_{m T+\nu},
$$

which admits a VARIMA $(1,1,0)$ representation

$$
\boldsymbol{\Psi}_{0} \Delta \mathbf{X}_{m}+\boldsymbol{\Psi}_{1} \Delta \mathbf{X}_{m-1}=\varepsilon_{m}
$$

where $\boldsymbol{\Psi}_{0}(r, s)=1$ for $r=s, \boldsymbol{\Psi}_{0}(r, s)=-\phi(s)$ for $r=s+1$ and $\boldsymbol{\Psi}_{0}(r, s)=0$ otherwise; $\boldsymbol{\Psi}_{1}(1, T)=-\phi(T)$ and $\Psi_{1}(r, s)=0$ otherwise, $1 \leq r, s \leq T$. Note that $\Delta \mathbf{X}_{m}=(1-B) \mathbf{X}_{m}=\chi_{1,1}^{(1)}\left(\mathbf{X}_{m}\right)$ in terms of GM increments. The spectral density of the one-step increment sequence $\Delta \mathbf{X}_{m}$ is the following:

$$
f(\lambda)=\frac{\lambda^{2}}{\left|1-e^{-i \lambda}\right|^{2}}\left|\Psi_{0}+\Psi_{1} e^{-i \lambda}\right|^{-2} .
$$

Consider the following estimation problem. Let us assume that we observe a time series $X_{m T+\nu}$ at points $m \leq-1,1 \leq \nu \leq T$. It is necessary to find an estimate $\widehat{A} X$ of the functional which depends on future values of $X_{m T+\nu}, m \geq 0,1 \leq \nu \leq T$, with a discount factor $\rho, 0<\rho<1$ :

$$
A X=\sum_{m=0}^{\infty} \sum_{\nu=1}^{T} \rho^{m T+\nu} X_{m T+\nu}=\sum_{m=0}^{\infty} \mathbf{a}_{m}^{\top} \mathbf{X}_{m}=: A \mathbf{X},
$$

where $\mathbf{a}_{m}=\rho^{m T}\left(\rho, \rho^{2}, \ldots, \rho^{T}\right)^{\top}=\rho^{m T} \mathbf{a}, \mathbf{a}=\left(\rho, \rho^{2}, \ldots, \rho^{T}\right)^{\top}$. Coefficients $\mathbf{b}_{m}, m \geq 0$ and $\mathbf{v}_{-1}$ from the representation $A \mathbf{X}=B \Delta \mathbf{X}-V \mathbf{X}$ are the following: $\mathbf{b}_{m}=\frac{\rho^{m T}}{1-\rho^{T}} \mathbf{a}, \mathbf{v}_{-1}=\mathbf{b}_{0}=-\frac{1}{1-\rho^{T}} \mathbf{a}$. Since $\left\|\mathbf{a}_{m}\right\|^{2}=$ $c_{1}(T) \rho^{m T},\left\|\mathbf{b}_{m}\right\|^{2}=c_{2}(T) \rho^{m T}$, conditions (21) and (22) are satisfied. Thus, we apply Theorem 3.3 to find the spectral characteristic of the estimate $\widehat{A} X$. We have

$$
\Phi_{(1)}\left(e^{-i \lambda}\right)=\Psi_{0}^{-1} \sum_{k=0}^{\infty}(-1)^{k}\left(\Psi_{1} \Psi_{0}^{-1}\right)^{k} e^{-i \lambda k}, \quad \Psi_{(1)}\left(e^{-i \lambda}\right)=\Psi_{0}+\Psi_{1} e^{-i \lambda},
$$


and

$$
\vec{r}_{(1)}\left(e^{i \lambda}\right)=\frac{1}{1-\rho^{T}} \Theta^{\top} \mathbf{a} \sum_{k=0}^{\infty} \rho^{k T} e^{i \lambda k},
$$

where $\Theta:=\left(\Psi_{0}+\rho^{T} \Psi_{1}\right)^{-1}$,

$$
\left(\Psi_{(1)}\left(e^{-i \lambda}\right)\right)^{\top} \vec{r}_{(1)}\left(e^{i \lambda}\right)=\frac{1}{1-\rho^{T}} \Psi_{1}^{\top} \Theta^{\top} \mathbf{a} e^{-i \lambda}+\frac{1}{1-\rho^{T}} \mathbf{a} \sum_{k=0}^{\infty} \rho^{k T} e^{i \lambda k},
$$

Then, the optimal estimate of the value of the functional $A X$ is calculated by the formula

$$
\widehat{A} X=-\frac{1}{1-\rho^{T}} \mathbf{a}^{\top} \Theta \Psi_{1} \Delta \mathbf{X}_{-1}+\frac{1}{1-\rho^{T}} \mathbf{a}^{\top} \mathbf{X}_{-1}=\frac{1}{1-\rho^{T}} \mathbf{a}^{\top}\left(\left(E_{T}-\Theta \Psi_{1}\right) \mathbf{X}_{-1}+\Theta \Psi_{1} \mathbf{X}_{-2}\right) .
$$

The value of the mean square error of the estimate is calculated by the formula

$$
\Delta(f ; \widehat{A} X)=\frac{1}{\left(1-\rho^{T}\right)^{3}\left(1+\rho^{T}\right)}\left\|\Theta^{\top} \mathbf{a}\right\|^{2} .
$$

\section{Example 5.2}

To illustrates a forecasting technique developed in Chapters 3 and 4 we consider a seasonal time series $x(t), t \in \mathbb{Z}$, exhibiting two fractional seasonal patterns and a periodic covariance behavior

$$
\left(1-B^{s}\right)^{d_{0}}\left(1-B^{u s}\right)^{d_{1}} \xi(t)=\varepsilon(t)-a_{0} \varepsilon(t-1)-a_{i(t)} \varepsilon(t-s), \quad i(t)=(t \quad \bmod s)+1,
$$

where $d_{0}=1+D_{0}, d_{1}=1+D_{1}, \varepsilon(t), t \in \mathbb{Z}$, are i.i.d. random variables with $\mathrm{E} \varepsilon(t)=0, \mathrm{E}|\varepsilon(t)|^{2}=1$. The first cycle $s$ may refer to 7 days within a week, and this pattern shows different correlation structure for each "season", namely, day of a week. The second seasonal pattern us may describe a year period assuming that $u=52$ corresponds to weeks within a year. Under the conditions stated below, the increment $w(t)=\left(1-B^{s}\right)(1-$ $\left.B^{u s}\right) \xi(t)$ is cyclostationary since coefficients $a_{i(t)}$ are periodic with the period $T=s$.

Define the vector-valued sequences $\vec{\xi}(m)=\left(\xi_{1}(m), \xi_{2}(m), \ldots, \xi_{s}(m)\right)^{\top}$, where $\xi_{p}(m)=\xi(s m+p-1)$, and $\vec{\varepsilon}(m)=\left(\varepsilon_{1}(m), \varepsilon_{2}(m), \ldots, \varepsilon_{s}(m)\right)^{\top}$, where $\varepsilon_{p}(m)=\varepsilon(s m+p-1)$. Consider an increment function $\chi_{(1,1),(1, u)}^{(2)}(B)=(1-B)\left(1-B^{u}\right)$ with the step $\bar{\mu}=(1,1)$. The GM increment sequence $\chi_{(1,1),(1, u)}^{(2)}(\vec{\xi}(m))$ admits the representation

$$
\begin{gathered}
(1-B)^{D_{0}}\left(1-B^{u}\right)^{D_{1}} \chi_{(1,1),(1, u)}^{(2)}(\vec{\xi}(m))=\Phi_{0} \vec{\varepsilon}(m)+\Phi_{1} \vec{\varepsilon}(m-1), \\
\Phi_{0}=\left(\begin{array}{ccccc}
1 & 0 & 0 & \ldots & 0 \\
-a_{0} & 1 & 0 & \ldots & 0 \\
0 & -a_{0} & 1 & \ddots & 0 \\
\vdots & \vdots & \ddots & \ddots & \vdots \\
0 & 0 & 0 & \ldots & 1
\end{array}\right), \Phi_{1}=\left(\begin{array}{ccccc}
-a_{1} & 0 & 0 & \ldots & -a_{0} \\
0 & -a_{2} & 0 & \ldots & 0 \\
0 & 0 & -a_{3} & \ddots & 0 \\
\vdots & \vdots & \ddots & \ddots & \vdots \\
0 & 0 & 0 & \ldots & -a_{s}
\end{array}\right) .
\end{gathered}
$$

It is stationary under conditions $\left|D_{0}+D_{1}\right|<1 / 2,\left|D_{1}\right|<1 / 2$. For instance, if $d_{0}=0.7, d_{1}=1.2$, then $D_{0}=-0.3$, $D_{1}=0.2$ and the process exhibits a long-memory behavior.

The spectral density $f(\lambda)$ of the GM increment sequence $\chi_{(1,1),(1, d)}^{(2)} \vec{\xi}(m)$ is

$$
\begin{aligned}
f(\lambda) & =\frac{\left|\beta^{(2)}(i \lambda)\right|^{2}}{\left|\chi_{(1,1)}^{(2)}\left(e^{-i \lambda}\right)\right|^{2}\left|\chi_{(1,1)}^{(D)}\left(e^{-i \lambda}\right)\right|^{2}}\left|\Phi_{(1,1)}\left(e^{-i \lambda}\right)\right|^{2} \\
& =\frac{\lambda^{2} \prod_{k=-[u / 2]}^{[u / 2]}(\lambda-2 \pi k / u)^{2}}{\left|1-e^{-i \lambda}\right|^{2}\left|1-e^{-i u \lambda}\right|^{2}\left|1-e^{-i \lambda}\right|^{2 D_{0}}\left|1-e^{-i u \lambda}\right|^{2 D_{1}}}\left|\Phi_{0}+\Phi_{1} e^{-i \lambda}\right|^{2}
\end{aligned}
$$


where

$$
\begin{aligned}
\left(\chi_{(1,1)}^{(D)}\left(e^{-i \lambda}\right)\right)^{-1} & =\left(1-e^{-i \lambda}\right)^{-D_{0}}\left(1-e^{-i u \lambda}\right)^{-D_{1}} \\
& =\left(1-e^{-i \lambda}\right)^{-D_{0}-D_{1}} \prod_{k=1}^{[u / 2]}\left(1-2 \cos (2 \pi k / u) e^{-i \lambda}+e^{-2 i \lambda}\right)^{-\widetilde{D}_{k}} \\
& =\sum_{k=0}^{\infty} G_{k^{*}}^{+}(k) e^{-i \lambda k}=\left(\sum_{k=0}^{\infty} G_{k^{*}}^{-}(k) e^{-i \lambda k}\right)^{-1},
\end{aligned}
$$

$k^{*}=[u / 2]+1, G_{k^{*}}^{+}(m)$ and $G_{k^{*}}^{-}(m), m \geq 0$, are defined by (60) and (61) respectively, $\widetilde{D}_{k}=D_{1}$ for $1 \leq k \leq$ $[u / 2]-1, \widetilde{D}_{[u / 2]}=D_{1}$ for odd $u$ and $\widetilde{D}_{[u / 2]}=D_{1} / 2$ for even $u$. Note, $G_{k^{*}}^{+}(0)=G_{k^{*}}^{-}(0)=1$.

Let us find an estimate of a weighted sum of two average weekly values of the time series $\xi(t)$

$$
A_{2 s} \xi=\alpha\left(\frac{1}{s} \sum_{k=0}^{s-1} \xi(k)\right)+(1-\alpha)\left(\frac{1}{s} \sum_{k=s}^{2 s-1} \xi(k)\right)
$$

based on observations of $\xi(t)$ at points $t=-1,-2, \ldots$

In terms of the sequence $\vec{\xi}(m)$, the functional $A_{2 s} \xi$ is rewritten as

$$
A_{2} \vec{\xi}=(\vec{a}(0))^{\top} \vec{\xi}(0)+(\vec{a}(1))^{\top} \vec{\xi}(1),
$$

where $\vec{a}(0)=\left(\alpha s^{-1}, \alpha s^{-1}, \ldots, \alpha s^{-1}\right)^{\top}, \quad \vec{a}(1)=\left((1-\alpha) s^{-1},(1-\alpha) s^{-1}, \ldots,(1-\alpha) s^{-1}\right)^{\top}, \quad$ and admits a representation

$$
A_{2} \vec{\xi}=B_{2} \chi \vec{\xi}-V_{2} \vec{\xi}=(\vec{b}(0))^{\top} \chi(\vec{\xi}(0))+(\vec{b}(1))^{\top} \chi(\vec{\xi}(1))-\sum_{k=-u-1}^{-1}\left(\vec{v}_{2}(k)\right)^{\top} \vec{\xi}(k)
$$

Here $\quad \vec{b}(0)=\vec{a}(0)+\vec{a}(1)=\left(s^{-1}, s^{-1}, \ldots, s^{-1}\right)^{\top}=s^{-1} \overline{\mathbf{1}}, \quad \vec{b}(1)=\vec{a}(1)=\left((1-\alpha) s^{-1},(1-\alpha) s^{-1}, \ldots,(1-\right.$ $\left.\alpha) s^{-1}\right)^{\top}=(1-\alpha) s^{-1} \overline{\mathbf{1}} ; \quad$ and further $\quad \vec{v}_{2}(k)=0 \quad$ for $\quad k=-1,-2, \ldots,-u+2, \quad \vec{v}_{2}(-u+1)=-\vec{b}(1)=$ $-(1-\alpha) s^{-1} \overline{\mathbf{1}}, \quad \vec{v}_{2}(-u)=-\vec{b}(0)+\vec{b}(1)=-\alpha s^{-1} \overline{\mathbf{1}}, \quad \vec{v}_{2}(-u-1)=\vec{b}(0)=s^{-1} \overline{\mathbf{1}}$. By $\overline{\mathbf{1}}$ we denote a vector $(1,1, \ldots, 1)^{\top}$ of dimension $s$. Note that

$$
(1-B)^{-1}\left(1-B^{u}\right)^{-1}=\sum_{k=0}^{\infty} d(k) B^{k}=\sum_{k=0}^{\infty}\left(1+\left[\frac{k}{u}\right]\right) B^{k} .
$$

We find the spectral characteristic $\vec{h}_{(1,1), 2}(\lambda)$ of the estimate $\widehat{A}_{2} \vec{\xi}$ using Theorem 3.2 as well as remarks to Theorem 4.1. First we obtain

$$
\begin{aligned}
& \Phi_{(1,1)}\left(e^{-i \lambda}\right)=\sum_{k=0}^{\infty}\left(G_{k^{*}}^{+} * \Phi\right)(k) e^{-i \lambda k}=G_{k^{*}}^{+}(0) \Phi_{0}+\sum_{k=1}^{\infty}\left(G_{k^{*}}^{+}(k) \Phi_{0}+G_{k^{*}}^{+}(k-1) \Phi_{1}\right) e^{-i \lambda k}, \\
& \Psi_{(1,1)}\left(e^{-i \lambda}\right)=\sum_{k=0}^{\infty}\left(G_{k^{*}}^{-} * \Psi\right)(k) e^{-i \lambda k}=\Phi_{0}^{-1} \sum_{k=0}^{\infty}\left(G_{k^{*}}^{-} * \widetilde{\Psi}\right)(k) e^{-i \lambda k},
\end{aligned}
$$

where $\left(G_{k^{*}}^{-} * \Psi\right)(k), k \geq 0$, is a convolution of two sequences $G_{k^{*}}^{-}(k)$ and $\Psi_{k}, k \geq 0, \Psi_{k}=(-1)^{k} \Phi_{0}^{-1}\left(\Phi_{1} \Phi_{0}^{-1}\right)^{k}$, $\widetilde{\Psi}_{k}=\Phi_{0}^{-1} \Psi_{k}=(-1)^{k}\left(\Phi_{1} \Phi_{0}^{-1}\right)^{k}$,

$$
\Phi_{1} \Phi_{0}^{-1}=\left(\begin{array}{ccccc}
-a_{1}-a_{0}^{s} & -a_{0}^{s-1} & -a_{0}^{s-2} & \ldots & -a_{0} \\
-a_{2} a_{0} & -a_{2} & 0 & \ldots & 0 \\
-a_{3} a_{0}^{2} & -a_{3} a_{0} & -a_{3} & \ldots & 0 \\
\vdots & \vdots & \vdots & \ddots & \vdots \\
-a_{s} a_{0}^{s-1} & -a_{s} a_{0}^{s-2} & -a_{s} a_{0}^{s-3} & \ldots & -a_{s}
\end{array}\right) .
$$


Then

$$
\begin{aligned}
\left(\Psi_{(1,1)}\left(e^{-i \lambda}\right)\right)^{\top} \vec{r}_{(1,1), 2}\left(e^{i \lambda}\right)= & \vec{b}(1) e^{i \lambda}+\vec{b}(0) \\
& +\sum_{k=1}^{\infty}\left(\left(G_{k^{*}}^{-} * \widetilde{\Psi}^{\top}\right)(k)\left(\vec{b}(0)+G_{k^{*}}^{+}(1) \vec{b}(1)\right)+G_{k^{*}}^{-}(k+1) \vec{b}(1)\right) e^{-i \lambda k} \\
= & \chi_{(1, u)}^{(D)}\left(e^{-i \lambda}\right)\left(\widetilde{\Psi}\left(e^{-i \lambda}\right)\right)^{\top}\left(\vec{b}(0)+D_{0} \vec{b}(1)\right)+\vec{b}(1) e^{i \lambda} \chi_{(1, u)}^{(D)}\left(e^{-i \lambda}\right),
\end{aligned}
$$

and

$$
\begin{aligned}
\vec{h}_{(1,1), 2}(\lambda) & =-\frac{\chi_{(1,1)}^{(2)}\left(e^{-i \lambda}\right)}{\beta^{(2)}(i \lambda)} \sum_{k=1}^{\infty} s^{-1}\left(\left(1+(1-\alpha) D_{0}\right)\left(G_{k^{*}}^{-} * \widetilde{\Psi}^{\top}\right)(k)+(1-\alpha) G_{k^{*}}^{-}(k+1) E_{T}\right) \overline{\mathbf{1}} e^{-i \lambda k} \\
& =\frac{\chi_{(1,1)}^{(2)}\left(e^{-i \lambda}\right)}{\beta^{(2)}(i \lambda)} \sum_{k=1}^{\infty} h(k) .
\end{aligned}
$$

The optimal estimate of the functional $A_{2 s} \xi$ is calculated by the formula

$$
\begin{aligned}
\widehat{A}_{2} \vec{\xi}= & -s^{-1}(\overline{\mathbf{1}})^{\top} \vec{\xi}(-u-1)+\alpha s^{-1}(\overline{\mathbf{1}})^{\top} \vec{\xi}(-u)+(1-\alpha) s^{-1}(\overline{\mathbf{1}})^{\top} \vec{\xi}(-u+1) \\
& +\sum_{k=1}^{\infty} h^{\top}(k)(\vec{\xi}(-k)-\vec{\xi}(-k-1)-\vec{\xi}(-k-u)+\vec{\xi}(-k-u-1)) .
\end{aligned}
$$

The value of the mean square error of the estimate is calculated by the formula

$$
\Delta\left(f ; \widehat{A}_{2} \vec{\xi}\right)=s^{-2}\left\|\left(\Phi_{0}+(1-\alpha)\left(D_{0} \Phi_{0}+\Phi_{1}\right)\right)^{\top} \overline{\mathbf{1}}\right\|^{2}+(1-\alpha)^{2} s^{-2}\left\|\Phi_{0}^{\top} \overline{\mathbf{1}}\right\|^{2} .
$$

In a particular case $d_{0}=1, d_{1}=1$ and, respectively, $D_{0}=0, D_{1}=0$, we have $\chi_{(1, u)}^{(D)}\left(e^{-i \lambda}\right) \equiv 1$, and $G_{k^{*}}^{ \pm}(k)=0$ for $k \geq 1$. In this case the estimate of the functional $A_{2 s} \xi$ and the value of the its mean square error are calculated by the formulas

$$
\begin{aligned}
\widehat{A}_{2} \vec{\xi}= & -s^{-1}(\overline{\mathbf{1}})^{\top} \vec{\xi}(-u-1)+\alpha s^{-1}(\overline{\mathbf{1}})^{\top} \vec{\xi}(-u)+(1-\alpha) s^{-1}(\overline{\mathbf{1}})^{\top} \vec{\xi}(-u+1) \\
& +s^{-1} \sum_{k=1}^{\infty}(-1)^{k+1}(\overline{\mathbf{1}})^{\top}\left(\Phi_{1} \Phi_{0}^{-1}\right)^{k}(\vec{\xi}(-k)-\vec{\xi}(-k-1)-\vec{\xi}(-k-u)+\vec{\xi}(-k-u-1)),
\end{aligned}
$$

and

$$
\Delta\left(f ; \widehat{A}_{2} \vec{\xi}\right)=s^{-2}\left(\sum_{k=1}^{s}\left(1-(1-\alpha)^{\delta_{k s}} a_{0}-(1-\alpha) a_{k}\right)^{2}+(1-\alpha)^{2}(s-1)\left(1-a_{0}\right)^{2}+(1-\alpha)^{2}\right) .
$$

\section{Minimax (robust) method of forecasting}

Values of the mean square errors and spectral characteristics of the optimal estimates of functionals $A \vec{\xi}$ and $A_{N} \vec{\xi}$ constructed from unobserved values of stochastic sequence $\vec{\xi}(m)$ which determine a stationary stochastic GM increment sequence $\chi_{\bar{\mu}, \bar{s}}^{(d)}(\vec{\xi}(m))$ with the spectral density matrix $f(\lambda)$ based on its observations $\vec{\xi}(m)$ at points $m=-1,-2, \ldots$ can be calculated by formulas (37), (36) and (43), (42) respectively, in the case where the spectral density matrix $f(\lambda)$ is exactly known. In the case where the spectral density $f(\lambda)$ admits the canonical factorization (38), formulas (71), (39) and (45), (44) are derived for calculating values of the mean square errors and spectral characteristics, respectively. 
In many practical cases, however, complete information about the spectral density matrix is impossible while some sets $\mathcal{D}$ of admissible spectral densities can be defined. In this case the minimax method of estimation of functionals from unobserved values of stochastic sequences is reasonable. This method consists in finding an estimate that minimizes the maximal values of the mean square errors for all spectral densities from a given class $\mathcal{D}$ of admissible spectral densities simultaneously.

\section{Definition 6.1}

For a given class of spectral densities $\mathcal{D}$ a spectral density $f_{0}(\lambda) \in \mathcal{D}$ is called the least favourable in $\mathcal{D}$ for the optimal linear estimation of the functional $A \vec{\xi}$ if the following relation holds true:

$$
\Delta\left(f_{0}\right)=\Delta\left(h_{\bar{\mu}}\left(f_{0}\right) ; f_{0}\right)=\max _{f \in \mathcal{D}} \Delta\left(h_{\bar{\mu}}(f) ; f\right) .
$$

\section{Definition 6.2}

For a given class of spectral densities $\mathcal{D}$ a spectral characteristic $h^{0}(\lambda)$ of the optimal linear estimate of the functional $A \xi$ is called minimax-robust if the following conditions are satisfied

$$
h^{0}(\lambda) \in H_{\mathcal{D}}=\bigcap_{f \in \mathcal{D}} L_{2}^{0-}(f), \quad \min _{h \in H_{\mathcal{D}}} \max _{f \in \mathcal{D}} \Delta(h ; f)=\sup _{f \in \mathcal{D}} \Delta\left(h^{0} ; f\right)
$$

Taking into account the introduced definitions and the relations derived in the previous sections we can verify that the following lemmas hold true.

\section{Lemma 6.1}

A spectral density $f_{0}(\lambda) \in \mathcal{D}$ satisfying the minimality condition (23) is the least favourable density in the class $\mathcal{D}$ for the optimal linear estimation of the functional $A \vec{\xi}$ based on observations of the sequence $\vec{\xi}(m)$ at points $m=-1,-2, \ldots$ if the operator $\mathbf{F}_{\bar{\mu}}^{0}$ defined by the Fourier coefficients of the function

$$
\left|\beta^{(d)}(i \lambda)\right|^{2}\left|\chi_{\bar{\mu}}^{(d)}\left(e^{-i \lambda}\right)\right|^{-2} f_{0}^{-1}(\lambda),
$$

determines a solution to the constrained optimization problem

$$
\max _{f \in \mathcal{D}}\left(\left\langle D^{\bar{\mu}} \mathbf{a}, \mathbf{F}_{\bar{\mu}}^{-1} D^{\bar{\mu}} \mathbf{a}\right\rangle\right)=\left\langle D^{\bar{\mu}} \mathbf{a},\left(\mathbf{F}_{\bar{\mu}}^{0}\right)^{-1} D^{\bar{\mu}} \mathbf{a}\right\rangle .
$$

The minimax spectral characteristic $h^{0}=h_{\bar{\mu}}\left(f^{0}\right)$ is calculated by formula (36) if $h_{\bar{\mu}}\left(f^{0}\right) \in H_{\mathcal{D}}$.

\section{Lemma 6.2}

A spectral density $f_{0}(\lambda) \in \mathcal{D}$ which admits the canonical factorization (38) is the least favourable density in the class $\mathcal{D}$ for the optimal linear estimation of the functional $A \vec{\xi}$ based on observations of the sequence $\vec{\xi}(m)$ at points $m=-1,-2, \ldots$ if coefficients $\left\{\varphi^{0}(k): k \geq 0\right\}$ of the canonical factorization

$$
f_{0}(\lambda)=\left(\sum_{k=0}^{\infty} \varphi^{0}(k) e^{-i \lambda k}\right)\left(\sum_{k=0}^{\infty} \varphi^{0}(k) e^{-i \lambda k}\right)^{*}
$$

of the spectral density $f^{0}(\lambda)$ determine a solution to the constrained optimization problem

$$
\left\|D^{\bar{\mu}} \mathbf{A} \varphi_{\bar{\mu}}\right\|^{2} \rightarrow \max , f(\lambda)=\left(\sum_{k=0}^{\infty} \varphi(k) e^{-i \lambda k}\right)\left(\sum_{k=0}^{\infty} \varphi(k) e^{-i \lambda k}\right)^{*} \in \mathcal{D} .
$$

The minimax spectral characteristic $h^{0}=h_{\bar{\mu}}\left(f_{0}\right)$ is calculated by formula (39) if $h_{\bar{\mu}}\left(f^{0}\right) \in H_{\mathcal{D}}$.

Lemma 6.3

A spectral density $f_{0}(\lambda) \in \mathcal{D}$ which admits the canonical factorization (38) is the least favourable density in the 
class $\mathcal{D}$ for the optimal linear extrapolation of the functional $A_{N} \vec{\xi}$ based on observations of the sequence $\vec{\xi}(m)$ at points $m=-1,-2, \ldots$ if coefficients $\left\{\varphi^{0}(k): k=0,1, \ldots, N\right\}$ from the canonical factorization

$$
f_{0}(\lambda)=\left(\sum_{k=0}^{N} \varphi^{0}(k) e^{-i \lambda k}\right)\left(\sum_{k=0}^{N} \varphi^{0}(k) e^{-i \lambda k}\right)^{*}
$$

of the spectral density $f_{0}(\lambda)$ determine a solution to the constrained optimization problem

$$
\left\|D_{N}^{\bar{\mu}} \mathbf{A}_{N} \varphi_{\mu, N}\right\|^{2} \rightarrow \max , f(\lambda)=\left(\sum_{k=0}^{N} \varphi(k) e^{-i \lambda k}\right)\left(\sum_{k=0}^{N} \varphi(k) e^{-i \lambda k}\right)^{*} \in \mathcal{D} .
$$

The minimax spectral characteristic $h^{0}=h_{\bar{\mu}}\left(f_{0}\right)$ is calculated by formula (44) if $h_{\mu, N}\left(f^{0}\right) \in H_{\mathcal{D}}$.

For more detailed analysis of properties of the least favorable spectral densities and the minimax-robust spectral characteristics we observe that the minimax spectral characteristic $h^{0}$ and the least favourable spectral density $f_{0}$ form a saddle point of the function $\Delta(h ; f)$ on the set $H_{\mathcal{D}} \times \mathcal{D}$. The saddle point inequalities

$$
\Delta\left(h ; f_{0}\right) \geq \Delta\left(h^{0} ; f_{0}\right) \geq \Delta\left(h^{0} ; f\right) \quad \forall f \in \mathcal{D}, \forall h \in H_{\mathcal{D}}
$$

hold true if $h^{0}=h_{\bar{\mu}}\left(f_{0}\right), h_{\bar{\mu}}\left(f_{0}\right) \in H_{\mathcal{D}}$ and $f_{0}$ is a solution of the constrained optimization problem

$$
\widetilde{\Delta}(f)=-\Delta\left(h_{\bar{\mu}}\left(f_{0}\right) ; f\right) \rightarrow \inf , \quad f \in \mathcal{D},
$$

where the functional $\Delta\left(h_{\bar{\mu}}\left(f_{0}\right) ; f\right)$ is calculated by the formula

$$
\begin{aligned}
& \Delta\left(h_{\bar{\mu}}\left(f_{0}\right) ; f\right)=\frac{1}{2 \pi} \int_{-\pi}^{\pi} \frac{\overline{\beta^{(d)}(i \lambda)}}{\overline{\chi_{\bar{\mu}}^{(d)}\left(e^{-i \lambda}\right)}}\left(\sum_{k=0}^{\infty}\left(\left(\mathbf{F}_{\bar{\mu}}^{0}\right)^{-1} D^{\bar{\mu}} \mathbf{a}\right)_{k} e^{i \lambda k}\right)^{\top} f_{0}^{-1}(\lambda) f(\lambda)
\end{aligned}
$$

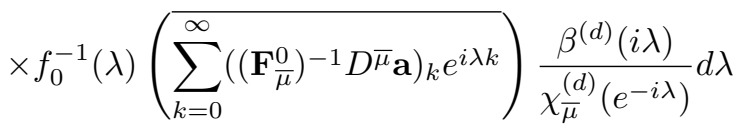

or by the formula

$$
\begin{aligned}
\Delta\left(h_{\bar{\mu}}\left(f_{0}\right) ; f\right)= & \frac{1}{2 \pi} \int_{-\pi}^{\pi} \frac{\chi_{\bar{\mu}}^{(d)}\left(e^{-i \lambda}\right)}{\beta^{(d)}(i \lambda)}\left(\sum_{k=0}^{\infty}\left(D^{\bar{\mu}} \mathbf{A} \varphi_{\bar{\mu}}^{0}\right)_{k} e^{i \lambda k}\right)^{\top}\left(\Psi_{\bar{\mu}}^{0}\left(e^{-i \lambda}\right)\right) f(\lambda) \\
& \times\left(\Psi_{\bar{\mu}}^{0}\left(e^{-i \lambda}\right)\right)^{*}\left(\sum_{k=0}^{\infty}\left(D^{\bar{\mu}} \mathbf{A} \varphi_{\bar{\mu}}^{0}\right)_{k} e^{i \lambda k}\right) \frac{\chi_{\bar{\mu}}^{(d)}\left(e^{-i \lambda}\right)}{\overline{\beta^{(d)}(i \lambda)}} d \lambda
\end{aligned}
$$

in the case where the spectral density admits the canonical factorization (38).

The constrained optimization problem (69) is equivalent to the unconstrained optimization problem

$$
\Delta_{\mathcal{D}}(f)=\widetilde{\Delta}(f)+\delta(f \mid \mathcal{D}) \rightarrow \inf ,
$$

where $\delta(f \mid \mathcal{D})$ is the indicator function of the set $\mathcal{D}$, namely $\delta(f \mid \mathcal{D})=0$ if $f \in \mathcal{D}$ and $\delta(f \mid \mathcal{D})=+\infty$ if $f \notin \mathcal{D}$. A solution $f_{0}$ of this unconstrained optimization problem is characterized by the condition $0 \in \partial \Delta_{\mathcal{D}}\left(f_{0}\right)$, which is the necessary and sufficient condition under which a point $f_{0}$ belongs to the set of minimums of the convex functional $\Delta_{\mathcal{D}}(f)[10,35,33,46]$. This condition makes it possible to find the least favourable spectral densities in some special classes of spectral densities $D$.

The form of the functional $\widetilde{\Delta}(f)$ allows us to apply the Lagrange method of indefinite multipliers for investigating the constrained optimization problem (69). The complexity of optimization problem is determined by the complexity of calculating the subdifferentials of the indicator functions of sets of admissible spectral densities. 


\subsection{Least favorable spectral density in classes $\mathcal{D}_{0}$}

Consider the forecasting problem for the functional $A \vec{\xi}$ which depends on unobserved values of a sequence $\vec{\xi}(m)$ with stationary GM increments based on observations of the sequence at points $m=-1,-2, \ldots$ under the condition that sets of admissible spectral densities $\mathcal{D}_{0}^{k}, k=1,2,3,4$ are defined as follows:

$$
\begin{gathered}
\mathcal{D}_{0}^{1}=\left\{f(\lambda) \mid \frac{1}{2 \pi} \int_{-\pi}^{\pi} \frac{\left|\chi_{\bar{\mu}}^{(d)}\left(e^{-i \lambda}\right)\right|^{2}}{\left|\beta^{(d)}(i \lambda)\right|^{2}} f(\lambda) d \lambda=P\right\}, \\
\mathcal{D}_{0}^{2}=\left\{f(\lambda) \mid \frac{1}{2 \pi} \int_{-\pi}^{\pi} \frac{\left|\chi_{\bar{\mu}}^{(d)}\left(e^{-i \lambda}\right)\right|^{2}}{\left|\beta^{(d)}(i \lambda)\right|^{2}} \operatorname{Tr}[f(\lambda)] d \lambda=p\right\}, \\
\mathcal{D}_{0}^{3}=\left\{f(\lambda) \mid \frac{1}{2 \pi} \int_{-\pi}^{\pi} \frac{\left|\chi_{\bar{\mu}}^{(d)}\left(e^{-i \lambda}\right)\right|^{2}}{\left|\beta^{(d)}(i \lambda)\right|^{2}} f_{k k}(\lambda) d \lambda=p_{k}, k=\overline{1, T}\right\}, \\
\mathcal{D}_{0}^{4}=\left\{f(\lambda) \mid \frac{1}{2 \pi} \int_{-\pi}^{\pi} \frac{\left|\chi_{\bar{\mu}}^{(d)}\left(e^{-i \lambda}\right)\right|^{2}}{\left|\beta^{(d)}(i \lambda)\right|^{2}}\left\langle B_{1}, f(\lambda)\right\rangle d \lambda=p\right\},
\end{gathered}
$$

where $p, p_{k}, k=\overline{1, T}$ are given numbers, $P, B_{1}$, are given positive-definite Hermitian matrices.

From the condition $0 \in \partial \Delta_{\mathcal{D}}\left(f_{0}\right)$ we find the following equations which determine the least favourable spectral densities for these given sets of admissible spectral densities.

For the first set of admissible spectral densities $\mathcal{D}_{0}^{1}$ we have equation

$$
\begin{aligned}
\left(\sum_{k=0}^{\infty}\left(\left(\mathbf{F}_{\bar{\mu}}^{0}\right)^{-1} D^{\bar{\mu}} \mathbf{a}\right)_{k} e^{i \lambda k}\right)\left(\sum_{k=0}^{\infty}\left(\left(\mathbf{F}_{\bar{\mu}}^{0}\right)^{-1} D^{\bar{\mu}} \mathbf{a}\right)_{k} e^{i \lambda k}\right)^{*}= \\
=\left(\frac{\left|\chi_{\bar{\mu}}^{(d)}\left(e^{-i \lambda}\right)\right|^{2}}{\left|\beta^{(d)}(i \lambda)\right|^{2}} f_{0}(\lambda)\right) \vec{\alpha} \cdot \vec{\alpha}^{*}\left(\frac{\left|\chi_{\bar{\mu}}^{(d)}\left(e^{-i \lambda}\right)\right|^{2}}{\left|\beta^{(d)}(i \lambda)\right|^{2}} f_{0}(\lambda)\right)
\end{aligned}
$$

where $\vec{\alpha}$ is a vector of Lagrange multipliers.

For the second set of admissible spectral densities $\mathcal{D}_{0}^{2}$ we have equation

$$
\left(\sum_{k=0}^{\infty}\left(\left(\mathbf{F}_{\bar{\mu}}^{0}\right)^{-1} D^{\bar{\mu}} \mathbf{a}\right)_{k} e^{i \lambda k}\right)\left(\sum_{k=0}^{\infty}\left(\left(\mathbf{F}_{\bar{\mu}}^{0}\right)^{-1} D^{\bar{\mu}} \mathbf{a}\right)_{k} e^{i \lambda k}\right)^{*}=\alpha^{2}\left(\frac{\left|\chi_{\bar{\mu}}^{(d)}\left(e^{-i \lambda}\right)\right|^{2}}{\left|\beta^{(d)}(i \lambda)\right|^{2}} f_{0}(\lambda)\right)^{2}
$$

where $\alpha^{2}$ is a Lagrange multiplier.

For the third set of admissible spectral densities $\mathcal{D}_{0}^{3}$ we have equation

$$
\begin{aligned}
\left(\sum_{k=0}^{\infty}\left(\left(\mathbf{F}_{\bar{\mu}}^{0}\right)^{-1} D^{\bar{\mu}} \mathbf{a}\right)_{k} e^{i \lambda k}\right)\left(\sum_{k=0}^{\infty}\left(\left(\mathbf{F}_{\bar{\mu}}^{0}\right)^{-1} D^{\bar{\mu}} \mathbf{a}\right)_{k} e^{i \lambda k}\right)^{*}= \\
=\left(\frac{\left|\chi_{\bar{\mu}}^{(d)}\left(e^{-i \lambda}\right)\right|^{2}}{\left|\beta^{(d)}(i \lambda)\right|^{2}} f_{0}(\lambda)\right)\left\{\alpha_{k}^{2} \delta_{k l}\right\}_{k, l=1}^{T}\left(\frac{\left|\chi_{\bar{\mu}}^{(d)}\left(e^{-i \lambda}\right)\right|^{2}}{\left|\beta^{(d)}(i \lambda)\right|^{2}} f_{0}(\lambda)\right)
\end{aligned}
$$

where $\alpha_{k}^{2}$ are Lagrange multipliers, $\delta_{k l}$ are Kronecker symbols. 
For the fourth set of admissible spectral densities $\mathcal{D}_{0}^{4}$ we have equation

$$
\begin{aligned}
\left(\sum_{k=0}^{\infty}\left(\left(\mathbf{F}_{\bar{\mu}}^{0}\right)^{-1} D^{\bar{\mu}} \mathbf{a}\right)_{k} e^{i \lambda k}\right)\left(\sum_{k=0}^{\infty}\left(\left(\mathbf{F}_{\bar{\mu}}^{0}\right)^{-1} D^{\bar{\mu}} \mathbf{a}\right)_{k} e^{i \lambda k}\right)^{*}= & \\
& =\alpha^{2}\left(\frac{\left|\chi_{\bar{\mu}}^{(d)}\left(e^{-i \lambda}\right)\right|^{2}}{\left|\beta^{(d)}(i \lambda)\right|^{2}} f_{0}(\lambda)\right) B_{1}^{\top}\left(\frac{\left|\chi_{\bar{\mu}}^{(d)}\left(e^{-i \lambda}\right)\right|^{2}}{\left|\beta^{(d)}(i \lambda)\right|^{2}} f_{0}(\lambda)\right)
\end{aligned}
$$

where $\alpha^{2}$ is a Lagrange multiplier.

In the case where the spectral density admits the canonical factorization (38) we have the following equations, correspondingly

$$
\begin{gathered}
\left(\sum_{k=0}^{\infty}\left(D^{\bar{\mu}} \mathbf{A} \varphi_{\bar{\mu}}^{0}\right)_{k} e^{i \lambda k}\right)\left(\sum_{k=0}^{\infty}\left(D^{\bar{\mu}} \mathbf{A} \varphi_{\bar{\mu}}^{0}\right)_{k} e^{i \lambda k}\right)^{*}=\left(\Phi_{\bar{\mu}}^{0}\left(e^{-i \lambda}\right)\right)^{\top} \vec{\alpha} \cdot \vec{\alpha}^{*} \overline{\Phi_{\bar{\mu}}^{0}\left(e^{-i \lambda}\right)} \\
\left(\sum_{k=0}^{\infty}\left(D^{\bar{\mu}} \mathbf{A} \varphi_{\bar{\mu}}^{0}\right)_{k} e^{i \lambda k}\right)\left(\sum_{k=0}^{\infty}\left(D^{\bar{\mu}} \mathbf{A} \varphi_{\bar{\mu}}^{0}\right)_{k} e^{i \lambda k}\right)^{*}=\alpha^{2}\left(\Phi_{\bar{\mu}}^{0}\left(e^{-i \lambda}\right)\right)^{\top} \overline{\Phi_{\bar{\mu}}^{0}\left(e^{-i \lambda}\right)} \\
\left(\sum_{k=0}^{\infty}\left(D^{\bar{\mu}} \mathbf{A} \varphi_{\bar{\mu}}^{0}\right)_{k} e^{i \lambda k}\right)\left(\sum_{k=0}^{\infty}\left(D^{\bar{\mu}} \mathbf{A} \varphi_{\bar{\mu}}^{0}\right)_{k} e^{i \lambda k}\right)^{*}=\left(\Phi_{\bar{\mu}}^{0}\left(e^{-i \lambda}\right)\right)^{\top}\left\{\alpha_{k}^{2} \delta_{k l}\right\}_{k, l=1}^{T} \overline{\Phi_{\bar{\mu}}^{0}\left(e^{-i \lambda}\right)}, \\
\left(\sum_{k=0}^{\infty}\left(D^{\bar{\mu}} \mathbf{A} \varphi_{\bar{\mu}}^{0}\right)_{k} e^{i \lambda k}\right)\left(\sum_{k=0}^{\infty}\left(D^{\bar{\mu}} \mathbf{A} \varphi_{\bar{\mu}}^{0}\right)_{k} e^{i \lambda k}\right)^{*}=\alpha^{2}\left(\Phi_{\bar{\mu}}^{0}\left(e^{-i \lambda}\right)\right)^{\top} B_{1}^{\top} \overline{\Phi_{\bar{\mu}}^{0}\left(e^{-i \lambda}\right)}
\end{gathered}
$$

The following theorem holds true.

Theorem 6.1

Let the minimality condition (23) hold true. The least favorable spectral densities $f_{0}(\lambda)$ in the classes $\mathcal{D}_{0}^{k}$, $k=1,2,3,4$, for the optimal linear estimation of the functional $A \vec{\xi}$ from observations of the sequence $\vec{\xi}(m)$ at points $m=-1,-2, \ldots$ are determined by equations (72), (73), (74), (75), (or equations (76), (77), (78), (79) in the case where the spectral densities admit the canonical factorization (38), respectively), the constrained optimization problem (64) and restrictions on densities from the corresponding classes $\mathcal{D}_{0}^{k}, k=1,2,3,4$. The minimax-robust spectral characteristic of the optimal estimate of the functional $A \vec{\xi}$ is determined by the formula (36).

\subsection{Least favorable spectral density in classes $\mathcal{D}_{V}^{U}$}

Consider the forecasting problem for the functional $A \vec{\xi}$ which depends on unobserved values of a sequence $\vec{\xi}(m)$ with stationary GM increments based on observations of the sequence at points $m=-1,-2, \ldots$ under the condition that sets of admissible spectral densities $\mathcal{D}_{V}^{U^{k}}, k=1,2,3,4$ are defined as follows:

$$
\begin{gathered}
\mathcal{D}_{V}^{U^{1}}=\left\{f(\lambda) \mid V(\lambda) \leq f(\lambda) \leq U(\lambda), \frac{1}{2 \pi} \int_{-\pi}^{\pi} \frac{\left|\chi_{\bar{\mu}}^{(d)}\left(e^{-i \lambda}\right)\right|^{2}}{\left|\beta^{(d)}(i \lambda)\right|^{2}} f(\lambda) d \lambda=Q\right\}, \\
\mathcal{D}_{V}^{U^{2}}=\left\{f(\lambda) \mid \operatorname{Tr}[V(\lambda)] \leq \operatorname{Tr}[f(\lambda)] \leq \operatorname{Tr}[U(\lambda)], \frac{1}{2 \pi} \int_{-\pi}^{\pi} \frac{\left|\chi_{\bar{\mu}}^{(d)}\left(e^{-i \lambda}\right)\right|^{2}}{\left|\beta^{(d)}(i \lambda)\right|^{2}} \operatorname{Tr}[f(\lambda)] d \lambda=q\right\}, \\
\mathcal{D}_{V}^{U^{3}}=\left\{f(\lambda) \mid v_{k k}(\lambda) \leq f_{k k}(\lambda) \leq u_{k k}(\lambda), \frac{1}{2 \pi} \int_{-\pi}^{\pi} \frac{\left|\chi_{\bar{\mu}}^{(d)}\left(e^{-i \lambda}\right)\right|^{2}}{\left|\beta^{(d)}(i \lambda)\right|^{2}} f_{k k}(\lambda) d \lambda=q_{k}, k=\overline{1, T}\right\}, \\
\mathcal{D}_{V}^{U^{4}}=\left\{f(\lambda) \mid\left\langle B_{2}, V(\lambda)\right\rangle \leq\left\langle B_{2}, f(\lambda)\right\rangle \leq\left\langle B_{2}, U(\lambda)\right\rangle, \frac{1}{2 \pi} \int_{-\pi}^{\pi} \frac{\left|\chi_{\bar{\mu}}^{(d)}\left(e^{-i \lambda}\right)\right|^{2}}{\left|\beta^{(d)}(i \lambda)\right|^{2}}\left\langle B_{2}, f(\lambda)\right\rangle d \lambda=q\right\} .
\end{gathered}
$$


Here the spectral densities $V(\lambda), U(\lambda)$ are known and fixed, $q, q_{k}, k=\overline{1, T}$ are given numbers, $Q, B_{2}$ are given positive definite Hermitian matrices.

From the condition $0 \in \partial \Delta_{\mathcal{D}}\left(f_{0}\right)$ we find the following equations which determine the least favourable spectral densities for these given sets of admissible spectral densities.

For the first set of admissible spectral densities $\mathcal{D}_{V}^{U^{1}}$ we have equation

$$
\begin{aligned}
\left(\sum_{k=0}^{\infty}\left(\left(\mathbf{F}_{\bar{\mu}}^{0}\right)^{-1} D^{\bar{\mu}} \mathbf{a}\right)_{k} e^{i \lambda k}\right) & \left(\sum_{k=0}^{\infty}\left(\left(\mathbf{F}_{\bar{\mu}}^{0}\right)^{-1} D^{\bar{\mu}} \mathbf{a}\right)_{k} e^{i \lambda k}\right)^{*}= \\
& =\left(\frac{\left|\chi_{\bar{\mu}}^{(d)}\left(e^{-i \lambda}\right)\right|^{2}}{\left|\beta^{(d)}(i \lambda)\right|^{2}} f_{0}(\lambda)\right)\left(\vec{\beta} \cdot \vec{\beta}^{*}+\Gamma_{1}(\lambda)+\Gamma_{2}(\lambda)\right)\left(\frac{\left|\chi_{\bar{\mu}}^{(d)}\left(e^{-i \lambda}\right)\right|^{2}}{\left|\beta^{(d)}(i \lambda)\right|^{2}} f_{0}(\lambda)\right)
\end{aligned}
$$

where $\vec{\beta}$ is a vector of Lagrange multipliers, $\Gamma_{1}(\lambda) \leq 0$ and $\Gamma_{1}(\lambda)=0$ if $f_{0}(\lambda)>V(\lambda), \Gamma_{2}(\lambda) \geq 0$ and $\Gamma_{2}(\lambda)=0$ if $f_{0}(\lambda)<U(\lambda)$.

For the second set of admissible spectral densities $\mathcal{D}_{V}^{U^{2}}$ we have equation

$$
\left(\sum_{k=0}^{\infty}\left(\left(\mathbf{F}_{\bar{\mu}}^{0}\right)^{-1} D^{\bar{\mu}} \mathbf{a}\right)_{k} e^{i \lambda k}\right)\left(\sum_{k=0}^{\infty}\left(\left(\mathbf{F}_{\bar{\mu}}^{0}\right)^{-1} D^{\bar{\mu}} \mathbf{a}\right)_{k} e^{i \lambda k}\right)^{*}=\left(\beta^{2}+\gamma_{1}(\lambda)+\gamma_{2}(\lambda)\right)\left(\frac{\left|\chi_{\bar{\mu}}^{(d)}\left(e^{-i \lambda}\right)\right|^{2}}{\left|\beta^{(d)}(i \lambda)\right|^{2}} f_{0}(\lambda)\right)^{2}
$$

where $\beta^{2}$ is Lagrange multiplier, $\gamma_{1}(\lambda) \leq 0$ and $\gamma_{1}(\lambda)=0$ if $\operatorname{Tr}\left[f_{0}(\lambda)\right]>\operatorname{Tr}[V(\lambda)], \gamma_{2}(\lambda) \geq 0$ and $\gamma_{2}(\lambda)=0$ if $\operatorname{Tr}\left[f_{0}(\lambda)\right]<\operatorname{Tr}[U(\lambda)]$.

For the third set of admissible spectral densities $\mathcal{D}_{V}^{U^{3}}$ we have equation

$$
\begin{aligned}
\left(\sum_{k=0}^{\infty}\left(\left(\mathbf{F}_{\bar{\mu}}^{0}\right)^{-1} D^{\bar{\mu}} \mathbf{a}\right)_{k} e^{i \lambda k}\right)\left(\sum_{k=0}^{\infty}\left(\left(\mathbf{F}_{\bar{\mu}}^{0}\right)^{-1} D^{\bar{\mu}} \mathbf{a}\right)_{k} e^{i \lambda k}\right)^{*}= \\
=\left(\frac{\left|\chi_{\bar{\mu}}^{(d)}\left(e^{-i \lambda}\right)\right|^{2}}{\left|\beta^{(d)}(i \lambda)\right|^{2}} f_{0}(\lambda)\right)\left\{\left(\beta_{k}^{2}+\gamma_{1 k}(\lambda)+\gamma_{2 k}(\lambda)\right) \delta_{k l}\right\}_{k, l=1}^{T}\left(\frac{\left|\chi_{\bar{\mu}}^{(d)}\left(e^{-i \lambda}\right)\right|^{2}}{\left|\beta^{(d)}(i \lambda)\right|^{2}} f_{0}(\lambda)\right),
\end{aligned}
$$

where $\beta_{k}^{2}$ are Lagrange multipliers, $\delta_{k l}$ are Kronecker symbols, $\gamma_{1 k}(\lambda) \leq 0$ and $\gamma_{1 k}(\lambda)=0$ if $f_{k k}^{0}(\lambda)>v_{k k}(\lambda)$, $\gamma_{2 k}(\lambda) \geq 0$ and $\gamma_{2 k}(\lambda)=0$ if $f_{k k}^{0}(\lambda)<u_{k k}(\lambda)$.

For the fourth set of admissible spectral densities $\mathcal{D}_{V}^{U^{4}}$ we have equation

$$
\begin{aligned}
\left(\sum_{k=0}^{\infty}\left(\left(\mathbf{F}_{\bar{\mu}}^{0}\right)^{-1} D^{\bar{\mu}} \mathbf{a}\right)_{k} e^{i \lambda k}\right) & \left(\sum_{k=0}^{\infty}\left(\left(\mathbf{F}_{\bar{\mu}}^{0}\right)^{-1} D^{\bar{\mu}} \mathbf{a}\right)_{k} e^{i \lambda k}\right)^{*}= \\
& =\left(\beta^{2}+\gamma_{1}^{\prime}(\lambda)+\gamma_{2}^{\prime}(\lambda)\right)\left(\frac{\left|\chi_{\bar{\mu}}^{(d)}\left(e^{-i \lambda}\right)\right|^{2}}{\left|\beta^{(d)}(i \lambda)\right|^{2}} f_{0}(\lambda)\right) B_{2}^{\top}\left(\frac{\left|\chi_{\bar{\mu}}^{(d)}\left(e^{-i \lambda}\right)\right|^{2}}{\left|\beta^{(d)}(i \lambda)\right|^{2}} f_{0}(\lambda)\right)
\end{aligned}
$$

where $\beta^{2}$ is Lagrange multiplier, $\gamma_{1}^{\prime}(\lambda) \leq 0$ and $\gamma_{1}^{\prime}(\lambda)=0$ if $\left\langle B_{2}, f_{0}(\lambda)\right\rangle>\left\langle B_{2}, V(\lambda)\right\rangle, \gamma_{2}^{\prime}(\lambda) \geq 0$ and $\gamma_{2}^{\prime}(\lambda)=0$ if $\left\langle B_{2}, f_{0}(\lambda)\right\rangle<\left\langle B_{2}, U(\lambda)\right\rangle$.

In the case where the spectral density admits the canonical factorization (38) we have the following equations, correspondingly

$$
\begin{gathered}
\left(\sum_{k=0}^{\infty}\left(D^{\bar{\mu}} \mathbf{A} \varphi_{\bar{\mu}}^{0}\right)_{k} e^{i \lambda k}\right)\left(\sum_{k=0}^{\infty}\left(D^{\bar{\mu}} \mathbf{A} \varphi_{\bar{\mu}}^{0}\right)_{k} e^{i \lambda k}\right)^{*}=\left(\Phi_{\bar{\mu}}^{0}\left(e^{-i \lambda}\right)\right)^{\top}\left(\vec{\beta} \cdot \vec{\beta}^{*}+\Gamma_{1}(\lambda)+\Gamma_{2}(\lambda)\right) \overline{\Phi_{\bar{\mu}}^{0}\left(e^{-i \lambda}\right)} \\
\left(\sum_{k=0}^{\infty}\left(D^{\bar{\mu}} \mathbf{A} \varphi_{\frac{0}{\mu}}^{0}\right)_{k} e^{i \lambda k}\right)\left(\sum_{k=0}^{\infty}\left(D^{\bar{\mu}} \mathbf{A} \varphi_{\frac{0}{\mu}}\right)_{k} e^{i \lambda k}\right)^{*}=\left(\beta^{2}+\gamma_{1}(\lambda)+\gamma_{2}(\lambda)\right)\left(\Phi_{\bar{\mu}}^{0}\left(e^{-i \lambda}\right)\right)^{\top} \overline{\Phi_{\bar{\mu}}^{0}\left(e^{-i \lambda}\right)}
\end{gathered}
$$




$$
\begin{aligned}
&\left(\sum_{k=0}^{\infty}\left(D^{\bar{\mu}} \mathbf{A} \varphi \frac{0}{\mu}\right)_{k} e^{i \lambda k}\right)\left(\sum_{k=0}^{\infty}\left(D^{\bar{\mu}} \mathbf{A} \varphi \frac{0}{\mu}\right)_{k} e^{i \lambda k}\right)^{*}==\left(\Phi_{\bar{\mu}}^{0}\left(e^{-i \lambda}\right)\right)^{\top}\left\{\left(\beta_{k}^{2}+\gamma_{1 k}(\lambda)+\gamma_{2 k}(\lambda)\right) \delta_{k l}\right\}_{k, l=1}^{T} \overline{\Phi_{\bar{\mu}}^{0}\left(e^{-i \lambda}\right)} \\
&\left(\sum_{k=0}^{\infty}\left(D^{\bar{\mu}} \mathbf{A} \varphi_{\bar{\mu}}^{0}\right)_{k} e^{i \lambda k}\right)\left(\sum_{k=0}^{\infty}\left(D^{\bar{\mu}} \mathbf{A} \varphi_{\bar{\mu}}^{0}\right)_{k} e^{i \lambda k}\right)^{*}=\left(\beta^{2}+\gamma_{1}^{\prime}(\lambda)+\gamma_{2}^{\prime}(\lambda)\right)\left(\Phi_{\bar{\mu}}^{0}\left(e^{-i \lambda}\right)\right)^{\top} B_{2}^{\top} \overline{\Phi_{\bar{\mu}}^{0}\left(e^{-i \lambda}\right)}
\end{aligned}
$$

The following theorem holds true.

\section{Theorem 6.2}

Let the minimality condition (23) hold true. The least favorable spectral densities $f_{0}(\lambda)$ in the classes $\mathcal{D}_{V}^{U^{k}}$, $k=1,2,3,4$, for the optimal linear estimation of the functional $A \vec{\xi}$ from observations of the sequence $\vec{\xi}(m)$ at points $m=-1,-2, \ldots$ are determined by equations (80), (81), (82), (83) (or equations (84), (85), (86), (87) in the case where the spectral densities admit the canonical factorization (38), respectively), the constrained optimization problem (64) and restrictions on densities from the corresponding classes $\mathcal{D}_{V}^{U^{k}}, k=1,2,3,4$. The minimax-robust spectral characteristic of the optimal estimate of the functional $A \vec{\xi}$ is determined by the formula (36).

\subsection{Least favorable spectral density in classes $\mathcal{D}_{\varepsilon}$}

Consider the forecasting problem for the functional $A \vec{\xi}$ which depends on unobserved values of a sequence $\vec{\xi}(m)$ with stationary GM increments based on observations of the sequence at points $m=-1,-2, \ldots$ under the condition that sets of admissible spectral densities $\mathcal{D}_{\varepsilon}^{k}, k=1,2,3,4$ are defined as follows:

$$
\begin{gathered}
\mathcal{D}_{\varepsilon}^{1}=\left\{f(\lambda) \mid \operatorname{Tr}[f(\lambda)]=(1-\varepsilon) \operatorname{Tr}\left[f_{1}(\lambda)\right]+\varepsilon \operatorname{Tr}[W(\lambda)], \frac{1}{2 \pi} \int_{-\pi}^{\pi} \frac{\left|\chi_{\bar{\mu}}^{(d)}\left(e^{-i \lambda}\right)\right|^{2}}{\left|\beta^{(d)}(i \lambda)\right|^{2}} \operatorname{Tr}[f(\lambda)] d \lambda=p\right\} ; \\
\mathcal{D}_{\varepsilon}^{2}=\left\{f(\lambda) \mid f_{k k}(\lambda)=(1-\varepsilon) f_{k k}^{1}(\lambda)+\varepsilon w_{k k}(\lambda), \frac{1}{2 \pi} \int_{-\pi}^{\pi} \frac{\left|\chi_{\bar{\mu}}^{(d)}\left(e^{-i \lambda}\right)\right|^{2}}{\left|\beta^{(d)}(i \lambda)\right|^{2}} f_{k k}(\lambda) d \lambda=p_{k}, k=\overline{1, T}\right\} ; \\
\mathcal{D}_{\varepsilon}^{3}=\left\{f(\lambda) \mid\left\langle B_{1}, f(\lambda)\right\rangle=(1-\varepsilon)\left\langle B_{1}, f_{1}(\lambda)\right\rangle+\varepsilon\left\langle B_{1}, W(\lambda)\right\rangle, \frac{1}{2 \pi} \int_{-\pi}^{\pi} \frac{\left|\chi_{\bar{\mu}}^{(d)}\left(e^{-i \lambda}\right)\right|^{2}}{\left|\beta^{(d)}(i \lambda)\right|^{2}}\left\langle B_{1}, f(\lambda)\right\rangle d \lambda=p\right\} ; \\
\mathcal{D}_{\varepsilon}^{4}=\left\{f(\lambda) \mid f(\lambda)=(1-\varepsilon) f_{1}(\lambda)+\varepsilon W(\lambda), \frac{1}{2 \pi} \int_{-\pi}^{\pi} \frac{\left|\chi_{\bar{\mu}}^{(d)}\left(e^{-i \lambda}\right)\right|^{2}}{\left|\beta^{(d)}(i \lambda)\right|^{2}} f(\lambda) d \lambda=P\right\} .
\end{gathered}
$$

Here $f_{1}(\lambda)$ is a fixed spectral density, $W(\lambda)$ is an unknown spectral density, $p, p_{k}, k=\overline{1, T}$, are given numbers, $P$ is a given positive-definite Hermitian matrices.

From the condition $0 \in \partial \Delta_{\mathcal{D}}\left(f_{0}\right)$ we find the following equations which determine the least favourable spectral densities for these given sets of admissible spectral densities.

For the first set of admissible spectral densities $\mathcal{D}_{\varepsilon}^{1}$ we have equation

$$
\left(\sum_{k=0}^{\infty}\left(\left(\mathbf{F}_{\bar{\mu}}^{0}\right)^{-1} D^{\bar{\mu}} \mathbf{a}\right)_{k} e^{i \lambda k}\right)\left(\sum_{k=0}^{\infty}\left(\left(\mathbf{F}_{\bar{\mu}}^{0}\right)^{-1} D^{\bar{\mu}} \mathbf{a}\right)_{k} e^{i \lambda k}\right)^{*}=\left(\alpha^{2}+\gamma_{1}(\lambda)\right)\left(\frac{\left|\chi_{\bar{\mu}}^{(d)}\left(e^{-i \lambda}\right)\right|^{2}}{\left|\beta^{(d)}(i \lambda)\right|^{2}} f_{0}(\lambda)\right)^{2},
$$

where $\alpha^{2}$ is Lagrange multiplier, $\gamma_{1}(\lambda) \leq 0$ and $\gamma_{1}(\lambda)=0$ if $\operatorname{Tr}\left[f_{0}(\lambda)\right]>(1-\varepsilon) \operatorname{Tr}\left[f_{1}(\lambda)\right]$.

For the second set of admissible spectral densities $\mathcal{D}_{\varepsilon}^{2}$ we have equation

$$
\begin{aligned}
&\left(\sum_{k=0}^{\infty}\left(\left(\mathbf{F}_{\bar{\mu}}^{0}\right)^{-1} D^{\bar{\mu}} \mathbf{a}\right)_{k} e^{i \lambda k}\right)\left(\sum_{k=0}^{\infty}\left(\left(\mathbf{F}_{\bar{\mu}}^{0}\right)^{-1} D^{\bar{\mu}} \mathbf{a}\right)_{k} e^{i \lambda k}\right)^{*}= \\
&=\left(\frac{\left|\chi_{\bar{\mu}}^{(d)}\left(e^{-i \lambda}\right)\right|^{2}}{\left|\beta^{(d)}(i \lambda)\right|^{2}} f_{0}(\lambda)\right)\left\{\left(\alpha_{k}^{2}+\gamma_{k}^{1}(\lambda)\right) \delta_{k l}\right\}_{k, l=1}^{T}\left(\frac{\left|\chi_{\bar{\mu}}^{(d)}\left(e^{-i \lambda}\right)\right|^{2}}{\left|\beta^{(d)}(i \lambda)\right|^{2}} f_{0}(\lambda)\right),
\end{aligned}
$$


where $\alpha_{k}^{2}$ are Lagrange multipliers, $\gamma_{k}^{1}(\lambda) \leq 0$ and $\gamma_{k}^{1}(\lambda)=0$ if $f_{k k}^{0}(\lambda)>(1-\varepsilon) f_{k k}^{1}(\lambda)$.

For the third set of admissible spectral densities $\mathcal{D}_{\varepsilon}^{3}$ we have equation

$$
\begin{aligned}
& \left(\sum_{k=0}^{\infty}\left(\left(\mathbf{F}_{\bar{\mu}}^{0}\right)^{-1} D^{\bar{\mu}} \mathbf{a}\right)_{k} e^{i \lambda k}\right)\left(\sum_{k=0}^{\infty}\left(\left(\mathbf{F}_{\bar{\mu}}^{0}\right)^{-1} D^{\bar{\mu}} \mathbf{a}\right)_{k} e^{i \lambda k}\right)^{*}= \\
& \quad=\left(\alpha^{2}+\gamma_{1}^{\prime}(\lambda)\right)\left(\frac{\left|\chi_{\bar{\mu}}^{(d)}\left(e^{-i \lambda}\right)\right|^{2}}{\left|\beta^{(d)}(i \lambda)\right|^{2}} f_{0}(\lambda)\right) B_{1}^{\top}\left(\frac{\left|\chi_{\bar{\mu}}^{(d)}\left(e^{-i \lambda}\right)\right|^{2}}{\left|\beta^{(d)}(i \lambda)\right|^{2}} f_{0}(\lambda)\right)
\end{aligned}
$$

where $\alpha^{2}$ is a Lagrange multiplier, $\gamma_{1}^{\prime}(\lambda) \leq 0$ and $\gamma_{1}^{\prime}(\lambda)=0$ if $\left\langle B_{1}, f_{0}(\lambda)\right\rangle>(1-\varepsilon)\left\langle B_{1}, f_{1}(\lambda)\right\rangle$.

For the fourth set of admissible spectral densities $\mathcal{D}_{\varepsilon}^{4}$ we have equation

$$
\begin{aligned}
\left(\sum_{k=0}^{\infty}\left(\left(\mathbf{F}_{\bar{\mu}}^{0}\right)^{-1} D^{\bar{\mu}} \mathbf{a}\right)_{k} e^{i \lambda k}\right)\left(\sum_{k=0}^{\infty}\left(\left(\mathbf{F}_{\bar{\mu}}^{0}\right)^{-1} D^{\bar{\mu}} \mathbf{a}\right)_{k} e^{i \lambda k}\right)^{*}= \\
=\left(\frac{\left|\chi_{\bar{\mu}}^{(d)}\left(e^{-i \lambda}\right)\right|^{2}}{\left|\beta^{(d)}(i \lambda)\right|^{2}} f_{0}(\lambda)\right)\left(\vec{\alpha} \cdot \vec{\alpha}^{*}+\Gamma(\lambda)\right)\left(\frac{\left|\chi_{\bar{\mu}}^{(d)}\left(e^{-i \lambda}\right)\right|^{2}}{\left|\beta^{(d)}(i \lambda)\right|^{2}} f_{0}(\lambda)\right),
\end{aligned}
$$

where $\vec{\alpha}$ is a vector of Lagrange multipliers, $\Gamma(\lambda) \leq 0$ and $\Gamma(\lambda)=0$ if $f_{0}(\lambda)>(1-\varepsilon) f_{1}(\lambda)$.

In the case where the spectral density admits the canonical factorization (38) we have the following equations, correspondingly

$$
\begin{gathered}
\left(\sum_{k=0}^{\infty}\left(D^{\bar{\mu}} \mathbf{A} \varphi \frac{0}{\mu}\right)_{k} e^{i \lambda k}\right)\left(\sum_{k=0}^{\infty}\left(D^{\bar{\mu}} \mathbf{A} \varphi \frac{0}{\mu}\right)_{k} e^{i \lambda k}\right)^{*}=\left(\alpha^{2}+\gamma_{1}(\lambda)\right)\left(\Phi_{\bar{\mu}}^{0}\left(e^{-i \lambda}\right)\right)^{\top} \overline{\Phi_{\bar{\mu}}^{0}\left(e^{-i \lambda}\right)} \\
\left(\sum_{k=0}^{\infty}\left(D^{\bar{\mu}} \mathbf{A} \varphi_{\bar{\mu}}^{0}\right)_{k} e^{i \lambda k}\right)\left(\sum_{k=0}^{\infty}\left(D^{\bar{\mu}} \mathbf{A} \varphi_{\bar{\mu}}^{0}\right)_{k} e^{i \lambda k}\right)^{*}=\left(\Phi_{\bar{\mu}}^{0}\left(e^{-i \lambda}\right)\right)^{\top}\left\{\left(\alpha_{k}^{2}+\gamma_{k}^{1}(\lambda)\right) \delta_{k l}\right\}_{k, l=1}^{T} \overline{\Phi_{\bar{\mu}}^{0}\left(e^{-i \lambda}\right)} \\
\left(\sum_{k=0}^{\infty}\left(D^{\bar{\mu}} \mathbf{A} \varphi_{\bar{\mu}}^{0}\right)_{k} e^{i \lambda k}\right)\left(\sum_{k=0}^{\infty}\left(D^{\bar{\mu}} \mathbf{A} \varphi_{\bar{\mu}}^{0}\right)_{k} e^{i \lambda k}\right)^{*}=\left(\alpha^{2}+\gamma_{1}^{\prime}(\lambda)\right)\left(\Phi_{\bar{\mu}}^{0}\left(e^{-i \lambda}\right)\right)^{\top} B_{1}^{\top} \overline{\Phi_{\bar{\mu}}^{0}\left(e^{-i \lambda}\right)} \\
\left(\sum_{k=0}^{\infty}\left(D^{\bar{\mu}} \mathbf{A} \varphi_{\bar{\mu}}^{0}\right)_{k} e^{i \lambda k}\right)\left(\sum_{k=0}^{\infty}\left(D^{\bar{\mu}} \mathbf{A} \varphi_{\bar{\mu}}^{0}\right)_{k} e^{i \lambda k}\right)^{*}=\left(\Phi_{\bar{\mu}}^{0}\left(e^{-i \lambda}\right)\right)^{\top}\left(\vec{\alpha} \cdot \vec{\alpha}^{*}+\Gamma(\lambda)\right) \overline{\Phi_{\bar{\mu}}^{0}\left(e^{-i \lambda}\right)}
\end{gathered}
$$

The following theorem holds true.

Theorem 6.3

Let the minimality condition (23) hold true. The least favorable spectral densities $f_{0}(\lambda)$ in the classes $\mathcal{D}_{\varepsilon}^{k}, k=$ $1,2,3,4$ for the optimal linear estimation of the functional $A \vec{\xi}$ from observations of the sequence $\vec{\xi}(m)$ at points $m=-1,-2, \ldots$ are determined by the equations (88), (89), (90), (91) (or equations (92), (93), (94), (95) in the case where the spectral densities admit the canonical factorization (38), respectively), the constrained optimization problem (64) and restrictions on densities from the corresponding classes $\mathcal{D}_{\varepsilon}^{k}, k=1,2,3,4$. The minimax-robust spectral characteristic of the optimal estimate of the functional $A \vec{\xi}$ is determined by the formula (36).

\subsection{Least favorable spectral density in classes $\mathcal{D}_{1 \delta}$}

Consider the forecasting problem for the functional $A \vec{\xi}$ which depends on unobserved values of a sequence $\vec{\xi}(m)$ with stationary GM increments based on observations of the sequence at points $m=-1,-2, \ldots$ under the 
condition that the sets of admissible spectral densities $\mathcal{D}_{1 \delta}^{k}, k=1,2,3,4$ are defined as follows:

$$
\begin{gathered}
\mathcal{D}_{1 \delta}^{1}=\left\{f(\lambda)\left|\frac{1}{2 \pi} \int_{-\pi}^{\pi} \frac{\left|\chi_{\bar{\mu}}^{(d)}\left(e^{-i \lambda}\right)\right|^{2}}{\left|\beta^{(d)}(i \lambda)\right|^{2}}\right| \operatorname{Tr}\left(f(\lambda)-f_{1}(\lambda)\right) \mid d \lambda \leq \delta\right\} ; \\
\mathcal{D}_{1 \delta}^{2}=\left\{f(\lambda)\left|\frac{1}{2 \pi} \int_{-\pi}^{\pi} \frac{\left|\chi_{\bar{\mu}}^{(d)}\left(e^{-i \lambda}\right)\right|^{2}}{\left|\beta^{(d)}(i \lambda)\right|^{2}}\right| f_{k k}(\lambda)-f_{k k}^{1}(\lambda) \mid d \lambda \leq \delta_{k}, k=\overline{1, T}\right\} ; \\
\mathcal{D}_{1 \delta}^{3}=\left\{f(\lambda)\left|\frac{1}{2 \pi} \int_{-\pi}^{\pi} \frac{\left|\chi_{\bar{\mu}}^{(d)}\left(e^{-i \lambda}\right)\right|^{2}}{\left|\beta^{(d)}(i \lambda)\right|^{2}}\right|\left\langle B_{2}, f(\lambda)-f_{1}(\lambda)\right\rangle \mid d \lambda \leq \delta\right\} ; \\
\mathcal{D}_{1 \delta}^{4}=\left\{f(\lambda)\left|\frac{1}{2 \pi} \int_{-\pi}^{\pi} \frac{\left|\chi_{\bar{\mu}}^{(d)}\left(e^{-i \lambda}\right)\right|^{2}}{\left|\beta^{(d)}(i \lambda)\right|^{2}}\right| f_{i j}(\lambda)-f_{i j}^{1}(\lambda) \mid d \lambda \leq \delta_{i}^{j}, i, j=\overline{1, T}\right\} .
\end{gathered}
$$

Here $f_{1}(\lambda)$ is a fixed spectral density, $\delta, \delta_{k}, k=\overline{1, T}, \delta_{i}^{j}, i, j=\overline{1, T}$, are given numbers.

From the condition $0 \in \partial \Delta_{\mathcal{D}}\left(f_{0}\right)$ we find the following equations which determine the least favourable spectral densities for these given sets of admissible spectral densities.

For the first set of admissible spectral densities $\mathcal{D}_{1 \delta}^{1}$ we have equation

$$
\begin{gathered}
\left(\sum_{k=0}^{\infty}\left(\left(\mathbf{F}_{\bar{\mu}}^{0}\right)^{-1} D^{\bar{\mu}} \mathbf{a}\right)_{k} e^{i \lambda k}\right)\left(\sum_{k=0}^{\infty}\left(\left(\mathbf{F}_{\bar{\mu}}^{0}\right)^{-1} D^{\bar{\mu}} \mathbf{a}\right)_{k} e^{i \lambda k}\right)^{*}=\beta^{2} \gamma_{2}(\lambda)\left(\frac{\left|\chi_{\bar{\mu}}^{(d)}\left(e^{-i \lambda}\right)\right|^{2}}{\left|\beta^{(d)}(i \lambda)\right|^{2}} f_{0}(\lambda)\right)^{2}, \\
\frac{1}{2 \pi} \int_{-\pi}^{\pi} \frac{\left|\chi_{\bar{\mu}}^{(d)}\left(e^{-i \lambda}\right)\right|^{2}}{\left|\beta^{(d)}(i \lambda)\right|^{2}}\left|\operatorname{Tr}\left(f_{0}(\lambda)-f_{1}(\lambda)\right)\right| d \lambda=\delta
\end{gathered}
$$

where $\beta^{2}$ is Lagrange multiplier, $\left|\gamma_{2}(\lambda)\right| \leq 1$ and

$$
\gamma_{2}(\lambda)=\operatorname{sign}\left(\operatorname{Tr}\left(f_{0}(\lambda)-f_{1}(\lambda)\right)\right): \operatorname{Tr}\left(f_{0}(\lambda)-f_{1}(\lambda)\right) \neq 0 .
$$

For the second set of admissible spectral densities $\mathcal{D}_{1 \delta}^{2}$ we have equation

$$
\begin{gathered}
\left(\sum_{k=0}^{\infty}\left(\left(\mathbf{F}_{\bar{\mu}}^{0}\right)^{-1} D^{\bar{\mu}} \mathbf{a}\right)_{k} e^{i \lambda k}\right)\left(\sum_{k=0}^{\infty}\left(\left(\mathbf{F}_{\bar{\mu}}^{0}\right)^{-1} D^{\bar{\mu}} \mathbf{a}\right)_{k} e^{i \lambda k}\right)^{*}= \\
=\left(\frac{\left|\chi_{\bar{\mu}}^{(d)}\left(e^{-i \lambda}\right)\right|^{2}}{\left|\beta^{(d)}(i \lambda)\right|^{2}} f_{0}(\lambda)\right)\left\{\beta_{k}^{2} \gamma_{k}^{2}(\lambda) \delta_{k l}\right\}_{k, l=1}^{T}\left(\frac{\left|\chi_{\bar{\mu}}^{(d)}\left(e^{-i \lambda}\right)\right|^{2}}{\left|\beta^{(d)}(i \lambda)\right|^{2}} f_{0}(\lambda)\right), \\
\frac{1}{2 \pi} \int_{-\pi}^{\pi} \frac{\left|\chi_{\bar{\mu}}^{(d)}\left(e^{-i \lambda}\right)\right|^{2}}{\left|\beta^{(d)}(i \lambda)\right|^{2}}\left|f_{k k}^{0}(\lambda)-f_{k k}^{1}(\lambda)\right| d \lambda=\delta_{k},
\end{gathered}
$$

where $\beta_{k}^{2}$ are Lagrange multipliers, $\left|\gamma_{k}^{2}(\lambda)\right| \leq 1$ and

$$
\gamma_{k}^{2}(\lambda)=\operatorname{sign}\left(f_{k k}^{0}(\lambda)-f_{k k}^{1}(\lambda)\right): f_{k k}^{0}(\lambda)-f_{k k}^{1}(\lambda) \neq 0, k=\overline{1, T}
$$

For the third set of admissible spectral densities $\mathcal{D}_{1 \delta}^{3}$ we have equation

$$
\begin{aligned}
& \left(\sum_{k=0}^{\infty}\left(\left(\mathbf{F}_{\bar{\mu}}^{0}\right)^{-1} D^{\bar{\mu}} \mathbf{a}\right)_{k} e^{i \lambda k}\right)\left(\sum_{k=0}^{\infty}\left(\left(\mathbf{F}_{\bar{\mu}}^{0}\right)^{-1} D^{\bar{\mu}} \mathbf{a}\right)_{k} e^{i \lambda k}\right)^{*}= \\
& \quad=\beta^{2} \gamma_{2}^{\prime}(\lambda)\left(\frac{\left|\chi_{\bar{\mu}}^{(d)}\left(e^{-i \lambda}\right)\right|^{2}}{\left|\beta^{(d)}(i \lambda)\right|^{2}} f_{0}(\lambda)\right) B_{2}^{\top}\left(\frac{\left|\chi_{\bar{\mu}}^{(d)}\left(e^{-i \lambda}\right)\right|^{2}}{\left|\beta^{(d)}(i \lambda)\right|^{2}} f_{0}(\lambda)\right),
\end{aligned}
$$




$$
\frac{1}{2 \pi} \int_{-\pi}^{\pi} \frac{\left|\chi_{\bar{\mu}}^{(d)}\left(e^{-i \lambda}\right)\right|^{2}}{\left|\beta^{(d)}(i \lambda)\right|^{2}}\left|\left\langle B_{2}, f_{0}(\lambda)-f_{1}(\lambda)\right\rangle\right| d \lambda=\delta,
$$

where $\beta^{2}$ is a Lagrange multiplier, $\left|\gamma_{2}^{\prime}(\lambda)\right| \leq 1$ and

$$
\gamma_{2}^{\prime}(\lambda)=\operatorname{sign}\left\langle B_{2}, f_{0}(\lambda)-f_{1}(\lambda)\right\rangle:\left\langle B_{2}, f_{0}(\lambda)-f_{1}(\lambda)\right\rangle \neq 0 .
$$

For the fourth set of admissible spectral densities $\mathcal{D}_{1 \delta}^{4}$ we have equation

$$
\begin{gathered}
\left(\sum_{k=0}^{\infty}\left(\left(\mathbf{F}_{\bar{\mu}}^{0}\right)^{-1} D^{\bar{\mu}} \mathbf{a}\right)_{k} e^{i \lambda k}\right)\left(\sum_{k=0}^{\infty}\left(\left(\mathbf{F}_{\bar{\mu}}^{0}\right)^{-1} D^{\bar{\mu}} \mathbf{a}\right)_{k} e^{i \lambda k}\right)^{*}= \\
=\left(\frac{\left|\chi_{\bar{\mu}}^{(d)}\left(e^{-i \lambda}\right)\right|^{2}}{\left|\beta^{(d)}(i \lambda)\right|^{2}} f_{0}(\lambda)\right)\left\{\beta_{i j}(\lambda) \gamma_{i j}(\lambda)\right\}_{i, j=1}^{T}\left(\frac{\left|\chi_{\bar{\mu}}^{(d)}\left(e^{-i \lambda}\right)\right|^{2}}{\left|\beta^{(d)}(i \lambda)\right|^{2}} f_{0}(\lambda)\right) \\
\frac{1}{2 \pi} \int_{-\pi}^{\pi} \frac{\left|\chi_{\bar{\mu}}^{(d)}\left(e^{-i \lambda}\right)\right|^{2}}{\left|\beta^{(d)}(i \lambda)\right|^{2}}\left|f_{i j}^{0}(\lambda)-f_{i j}^{1}(\lambda)\right| d \lambda=\delta_{i}^{j}
\end{gathered}
$$

where $\beta_{i j}$ are Lagrange multipliers, $\left|\gamma_{i j}(\lambda)\right| \leq 1$ and

$$
\gamma_{i j}(\lambda)=\frac{f_{i j}^{0}(\lambda)-f_{i j}^{1}(\lambda)}{\left|f_{i j}^{0}(\lambda)-f_{i j}^{1}(\lambda)\right|}: f_{i j}^{0}(\lambda)-f_{i j}^{1}(\lambda) \neq 0, i, j=\overline{1, T} .
$$

In the case where the spectral density admits the canonical factorization (38) we have the following equations, correspondingly

$$
\begin{gathered}
\left(\sum_{k=0}^{\infty}\left(D^{\bar{\mu}} \mathbf{A} \varphi_{\bar{\mu}}^{0}\right)_{k} e^{i \lambda k}\right)\left(\sum_{k=0}^{\infty}\left(D^{\bar{\mu}} \mathbf{A} \varphi_{\bar{\mu}}^{0}\right)_{k} e^{i \lambda k}\right)^{*}=\beta^{2} \gamma_{2}(\lambda)\left(\Phi_{\bar{\mu}}^{0}\left(e^{-i \lambda}\right)\right)^{\top} \overline{\Phi_{\bar{\mu}}^{0}\left(e^{-i \lambda}\right)} \\
\left(\sum_{k=0}^{\infty}\left(D^{\bar{\mu}} \mathbf{A} \varphi_{\bar{\mu}}^{0}\right)_{k} e^{i \lambda k}\right)\left(\sum_{k=0}^{\infty}\left(D^{\bar{\mu}} \mathbf{A} \varphi_{\bar{\mu}}^{0}\right)_{k} e^{i \lambda k}\right)^{*}=\left(\Phi_{\bar{\mu}}^{0}\left(e^{-i \lambda}\right)\right)^{\top}\left\{\beta_{k}^{2} \gamma_{k}^{2}(\lambda) \delta_{k l}\right\}_{k, l=1}^{T} \overline{\Phi_{\bar{\mu}}^{0}\left(e^{-i \lambda}\right)} \\
\left(\sum_{k=0}^{\infty}\left(D^{\bar{\mu}} \mathbf{A} \varphi_{\bar{\mu}}^{0}\right)_{k} e^{i \lambda k}\right)\left(\sum_{k=0}^{\infty}\left(D^{\bar{\mu}} \mathbf{A} \varphi_{\bar{\mu}}^{0}\right)_{k} e^{i \lambda k}\right)^{*}=\beta^{2} \gamma_{2}^{\prime}(\lambda)\left(\Phi_{\bar{\mu}}^{0}\left(e^{-i \lambda}\right)\right)^{\top} B_{2}^{\top} \overline{\Phi_{\bar{\mu}}^{0}\left(e^{-i \lambda}\right)} \\
\left(\sum_{k=0}^{\infty}\left(D^{\bar{\mu}} \mathbf{A} \varphi_{\bar{\mu}}^{0}\right)_{k} e^{i \lambda k}\right)\left(\sum_{k=0}^{\infty}\left(D^{\bar{\mu}} \mathbf{A} \varphi_{\bar{\mu}}^{0}\right)_{k} e^{i \lambda k}\right)^{*}=\left(\Phi_{\bar{\mu}}^{0}\left(e^{-i \lambda}\right)\right)^{\top}\left\{\beta_{i j}(\lambda) \gamma_{i j}(\lambda)\right\}_{i, j=1}^{T} \overline{\Phi_{\bar{\mu}}^{0}\left(e^{-i \lambda}\right)}
\end{gathered}
$$

The following theorem holds true.

\section{Theorem 6.4}

Let the minimality condition (23) hold true. The least favorable spectral densities $f_{0}(\lambda)$ in the classes $\mathcal{D}_{1 \delta}^{k}, k=$ $1,2,3,4$ for the optimal linear estimation of the functional $A \vec{\xi}$ from observations of the sequence $\vec{\xi}(m)$ at points $m=-1,-2, \ldots$ are determined by equations (96), (97); (98), (99); (100), (101); (102), (103) (or equations (104), (105), (106), (107) in the case where the spectral densities admit the canonical factorization (38), respectively), the constrained optimization problem (64) and restrictions on densities from the corresponding classes $\mathcal{D}_{1 \delta}^{k}, k=$ $1,2,3,4$. The minimax-robust spectral characteristic of the optimal estimate of the functional $A \vec{\xi}$ is determined by the formula (36). 


\section{Conclusions}

In this article, we present results of investigation of stochastic sequences with periodically stationary long memory multiple seasonal increments. We give definition of generalized multiple increment sequence and introduce stochastic sequences $\zeta(m)$ with periodically stationary (periodically correlated, cyclostationary) generalized multiple increments. These non-stationary stochastic sequences combine periodic structure of covariation functions of sequences as well as multiple seasonal factors, including the integrating one. A short review of the spectral theory of vector-valued generalized multiple increment sequences is presented. We describe methods of solution of the classical forecasting problem for linear functionals which are constructed from unobserved values of a sequence with periodically stationary generalized multiple increments in the case where the spectral structure of the sequence is exactly known. Estimates are obtained by representing the sequence under investigation as a vector-valued sequence with stationary generalized multiple increments and applying the Hilbert space projection technique. An approach to forecasting in the presence of non-stationary fractional integration is discussed. Examples of solution of the forecasting problem for particular models of time series are proposed. The minimax-robust approach to forecasting problem is applied in the case of spectral uncertainty where densities of sequences are not exactly known while, instead, sets of admissible spectral densities are specified. We propose a representation of the mean square error in the form of a linear functional in $L_{1}$ with respect to spectral densities, which allows us to solve the corresponding constrained optimization problem and describe the minimax (robust) estimates of the functionals. Relations are described which determine the least favourable spectral densities and the minimax spectral characteristics of the optimal estimates of linear functionals for a collection of specific classes of admissible spectral densities.

\section{Appendix}

Proof of Lemma 2.1

We have

$$
\begin{aligned}
\prod_{i=1}^{r}\left(1-B_{\mu_{i}}^{s_{i}}\right)^{d_{i}} & =\prod_{i=1}^{r}\left(\sum_{j_{i}=0}^{d_{i}}(-1)^{j_{i}}\left(\begin{array}{c}
d_{i} \\
j_{i}
\end{array}\right) B^{j_{i} \mu_{i} s_{i}}\right) \\
& =\prod_{i=1}^{r}\left(\sum_{j_{i}=0}^{d_{i} \mu_{i} s_{i}}(-1)^{\left[j_{i} / \mu_{i} s_{i}\right]}\left(\begin{array}{c}
d_{i} \\
\left.j_{i} / \mu_{i} s_{i}\right]
\end{array}\right) \mathbb{I}\left\{j_{i} \quad \bmod \mu_{i} s_{i}=0\right\} B^{j_{i}}\right) \\
& =\sum_{j_{1}=0}^{n_{1}} \ldots \sum_{j_{r}=0}^{n_{r}}\left((-1)^{\sum_{i=1}^{r} M_{i}^{j_{i}}} \prod_{i=1}^{r} I_{i}^{j_{i}} \prod_{i=1}^{r}\left(\begin{array}{c}
d_{i} \\
M_{i}^{j_{i}}
\end{array}\right)\right) B^{\sum_{i=1}^{r} j_{i}} .
\end{aligned}
$$


By replacing consequently $j_{1} \rightarrow k_{1}, k_{1}+j_{2} \rightarrow k_{2}, k_{2}+j_{3} \rightarrow k_{3}, \ldots, k_{r-1}+j_{r} \rightarrow k_{r}:=k$, we obtain

$$
\begin{aligned}
\prod_{i=1}^{r}\left(1-B_{\mu_{i}}^{s_{i}}\right)^{d_{i}}= & \sum_{k_{2}=0}^{n_{1}+n_{2}} \sum_{k_{1}=0 \vee k_{2}-n_{2}}^{n_{1} \wedge k_{2}} \sum_{j_{3}=0}^{n_{3}} \ldots \sum_{j_{r}=0}^{n_{r}}(-1)^{M_{2}^{k_{2}-k_{1}}+M_{1}^{k_{1}}} I_{2}^{k_{2}-k_{1}} I_{1}^{k_{1}} \\
& \times\left(\begin{array}{c}
d_{2} \\
M_{2}^{k_{2}-k_{1}}
\end{array}\right)\left(\begin{array}{c}
d_{1} \\
M_{1}^{k_{1}}
\end{array}\right)\left((-1)^{\sum_{i=3}^{r} M_{i}^{j_{i}}} \prod_{i=3}^{r} I_{i}^{j_{i}} \prod_{i=3}^{r}\left(\begin{array}{c}
d_{i} \\
M_{i}^{j_{i}}
\end{array}\right)\right) B^{k_{2}+\sum_{i=3}^{r} j_{i}} \\
= & \sum_{k_{3}=0}^{n_{1}+n_{2}+n_{3}} \sum_{k_{2}=0 \vee k_{3}-n_{3}}^{n_{1}+n_{2} \wedge k_{3}} \sum_{k_{1}=0 \vee k_{2}-n_{2}}^{n_{1} \wedge k_{2}} \sum_{j_{4}=0}^{n-4} \cdots \sum_{j_{r}=0}^{n_{r}}(-1)^{\sum_{i=1}^{3} M_{i}^{k_{i}-k_{i-1}}} \prod_{i=1}^{3} I_{i}^{k_{i}-k_{i-1}} \\
& \times \prod_{i=1}^{3}\left(\begin{array}{c}
d_{i} \\
M_{i}^{k_{i}-k_{i-1}}
\end{array}\right)\left((-1)^{\sum_{i=4}^{r} M_{i}^{j_{i}}} \prod_{i=3}^{r} I_{i}^{j_{i}} \prod_{i=3}^{r}\left(\begin{array}{c}
d_{i} \\
M_{i}^{j_{i}}
\end{array}\right)\right) B^{k_{3}+\sum_{i=4}^{r} j_{i}} \\
= & \sum_{k=0}^{n(\gamma)} e_{\gamma}(k) B^{k},
\end{aligned}
$$

where $e_{\gamma}(k)$ are coefficients from the lemma statement.

\section{Proof of Theorem 2.2}

We follow the idea proposed by Yaglom [51] for continuous time stationary increments. Consider a GM increment sequence with one seasonal factor $\chi_{\mu, s}^{(d)}(\eta(m))=\eta_{s}^{(d)}(m, \mu)=\left(1-B_{\mu}^{s}\right)^{d} \eta(m)$. Formula (8) implies

$$
c_{s}^{(d)}(\mu)=\mathrm{E} \eta_{s}^{(d)}(m, \mu)=\left(A_{0}+A_{1}+\ldots+A_{(\mu-1) d}\right) c_{s}^{(d)}(1)=\mu^{d} c_{s}^{(d)}(1)=c \mu^{d},
$$

where $c=c_{s}^{(d)}(1)$ does not depend on $\mu$.

Since $D_{s}^{(n)}(m ; \mu, \mu)$ is a positive-definite function with respect to variable $m$, one can define a function $F_{\mu, s}(\lambda)$ depending on the parameter $\mu$, which is a real bounded non-decreasing left-continuous with respect to $\lambda \in[-\pi, \pi)$ function, such that

$$
D_{s}^{(d)}(m ; \mu, \mu)=\int_{-\pi}^{\pi} e^{i \lambda m} d F_{\mu, s}(\lambda)
$$

Again, formula (8) implies

$$
\begin{aligned}
\mathrm{E} \eta_{s}^{(d)}\left(m_{1}+m, \mu\right) \overline{\eta_{s}^{(d)}(m, \mu)} & =\sum_{p=0}^{(\mu-1) d} \sum_{q=0}^{(\mu-1) d} A_{p} A_{q} \mathrm{E} \eta_{s}^{(d)}\left(m_{1}+m-p s, 1\right) \overline{\eta_{s}^{(d)}\left(m_{2}-q s, 1\right)} \\
& =\int_{-\pi}^{\pi} \sum_{p=0}^{(\mu-1) d(\mu-1) d} \sum_{q=0} A_{p} A_{q} e^{i \lambda(m-(p-q) s)} d F_{1, s}(\lambda) \\
& =\int_{-\pi}^{\pi} e^{i \lambda m} \sum_{p=0}^{(k-1) d} A_{p} e^{-i p s \lambda} \sum_{q=0}^{(\mu-1) d} A_{q} e^{i q s \lambda} d F_{1, s}(\lambda) \\
& =\int_{\pi}^{\pi} e^{i m \lambda} \frac{\left(1-e^{-i \mu s \lambda}\right)^{d}}{\left(1-e^{-i s \lambda}\right)^{d}} \frac{\left(1-e^{i \mu s \lambda}\right)^{d}}{\left(1-e^{i s \lambda}\right)^{d}} d F_{1, s}(\lambda) .
\end{aligned}
$$

Thus,

$$
\int_{\pi}^{\pi} e^{i m \lambda} d F_{\mu, s}(\lambda)=\int_{-\pi}^{\pi} e^{i m \lambda} \frac{(1-\cos \mu s \lambda)^{d}}{(1-\cos s \lambda)^{d}} F_{1, s}(\lambda) .
$$

The latter equality implies

$$
F_{\mu, s}(\lambda)=\int_{0}^{\lambda} \frac{(1-\cos \mu s u)^{d}}{(1-\cos s u)^{d}} F_{1, s}(u)
$$


and

$$
\int_{0}^{\lambda} \frac{\left|\beta^{(d)}(i u)\right|^{2}}{(1-\cos \mu s u)^{d}} d F_{\mu, s}(u)=\int_{0}^{\lambda} \frac{\left|\beta^{(d)}(i u)\right|^{2}}{(1-\cos s u)^{d}} d F_{1, s}(u),
$$

where the function $\beta^{(d)}(i u)$ is to be chosen in the way that the integrals are defined for $\lambda \in[-\pi, \pi)$ and converge at the neighborhoods of the points $\cos s u=1, u \in[-\pi, \pi]$, namely, the points $u=2 \pi k / s$ for $|k| \leq s / 2, k \in \mathbb{Z}$. Thus, we choose the function $\beta^{(d)}(i u)=\prod_{k=-[s / 2]}^{[s / 2]}(i u-2 \pi i k / s)^{d}$.

The right side of equality (110) doesn't depend on $k$, thus, put

$$
F(\lambda)=\frac{1}{2^{d}} \int_{0}^{\lambda} \frac{\left|\beta^{(d)}(i u)\right|^{2}}{(1-\cos \mu s u)^{d}} d F_{\mu, s}(u)
$$

The function $F(\lambda)$ is real-valued non-decreasing bounded function defined on $[-\pi, \pi)$, such that $F(0)=0$. Consider the function $F(\lambda)$ as left-continuous. This function is the one stated in the theorem.

Relation (11) for $r=1$ is obtained by considering the following equality for positive $\mu_{1}, \mu_{2}$ :

$$
\begin{aligned}
D_{s}^{(n)}\left(m, \mu_{1}, \mu_{2}\right) & =\int_{-\pi}^{\pi} e^{i m \lambda} \frac{\left(1-e^{-i \mu_{1} s \lambda}\right)^{d}}{\left(1-e^{-i s \lambda}\right)^{d}} \frac{\left(1-e^{i \mu_{2} s \lambda}\right)^{d}}{\left(1-e^{i s \lambda}\right)^{d}} d F_{1, s}(\lambda) \\
& =\int_{-\pi}^{\pi} e^{i m \lambda}\left(1-e^{-i \mu_{1} s \lambda}\right)^{d}\left(1-e^{i \mu_{2} s \lambda}\right)^{d} \frac{1}{\left|\beta^{(d)}(i \lambda)\right|^{2}} d F(\lambda) .
\end{aligned}
$$

For negative $\mu_{1}, \mu_{2}$, equality ( 7 ) is applied.

By generalizing for $r>1$ the given reasonings, we obtain the relations (10) and (11).

\section{Proof of Lemma 3.1}

Using Definition 2.3 we obtain the formal equality

$$
\xi_{p}(k)=\frac{1}{\left(1-B_{\mu}\right)^{n}} \chi_{\bar{\mu}, \bar{s}}^{(n)}\left(\xi_{p}(k)\right)=\sum_{j=-\infty}^{k} d_{\mu}(k-j) \chi_{\bar{\mu}, \bar{s}}^{(n)}\left(\xi_{p}(j)\right),
$$

which imply

$$
\begin{aligned}
\sum_{k=0}^{\infty} a_{p}(k) \xi_{p}(k) & =-\sum_{i=-n(\gamma)}^{-1} v_{p}(i) \xi_{p}(i)+\sum_{i=0}^{\infty}\left(\sum_{k=i}^{\infty} a_{p}(k) d_{\mu}(k-i)\right) \chi_{\bar{\mu}, \bar{s}}^{(n)}\left(\xi_{p}(i)\right), \\
\sum_{i=0}^{\infty} b_{p}(i) \chi_{\bar{\mu}, \bar{s}}^{(n)}\left(\xi_{p}(i)\right) & =\sum_{k=-n(\gamma)}^{-1} \xi_{p}(k) \sum_{l=0}^{k+n(\gamma)} e_{\nu}(l-k) b_{p}(k)+\sum_{k=0}^{\infty} \xi_{p}(k) \sum_{l=k}^{k+n(\gamma)} e_{\nu}(l-k) b_{p}(k),
\end{aligned}
$$

Relations (112) and (113) imply a representation $A \vec{\xi}=B \vec{\xi}-V \vec{\xi}$ and relations which prove the lemma:

$$
\begin{aligned}
& v_{p}(k)=\sum_{l=0}^{k+n(\gamma)} e_{\nu}(l-k) b_{p}(l), \quad k=-1,-2, \ldots,-n(\gamma), \quad p=1,2, \ldots, T, \\
& b_{p}(k)=\sum_{m=k}^{\infty} d_{\bar{\mu}}(m-k) a_{p}(m), \quad k=0,1,2, \ldots, \quad p=1,2, \ldots, T . \quad \square
\end{aligned}
$$

\section{REFERENCES}

1. J. Andel, Long memory time series models, Kybernetika, vol. 22, no. 2, pp.105-123, 1986. 
2. J. Arteche and P. Robinson, Semiparametric inference in seasonal and cyclical long-memory processes, Journal of Time Series Analysis, vol. 21, no. 1, pp. 1-?5, 2002.

3. C. Baek, R. A. Davis and V. Pipiras, Periodic dynamic factor models: estimation approaches and applications, Electronic Journal of Statistics, vol. 12, no. 2, pp. 4377-4411, 2018.

4. R. T. Baillie, C. Kongcharoen, and G. Kapetanios, Prediction from ARFIMA models: Comparisons between MLE and semiparametric estimation procedures, International Journal of Forecasting, vol. 28, pp. 46-?3, 2012.

5. I.V. Basawa, R. Lund and Q. Shao, First-order seasonal autoregressive processes with periodically varying parameters, Statistics \& Probability Letters, vol. 67, no. 4, pp. 299-306, 2004.

6. G. E. P. Box, G. M. Jenkins, G. C. Reinsel and G.M. Ljung, Time series analysis. Forecasting and control. 5rd ed., Hoboken, NJ: John Wiley \& Sons, 712 p., 2016.

7. I. I. Dubovets'ka and M. P. Moklyachuk, Extrapolation of periodically correlated processes from observations with noise, Theory of Probability and Mathematical Statistics, vol. 88, pp. 67-83, 2014.

8. G. Dudek, Forecasting time series with multiple seasonal cycles using neural networks with local learning, In: Rutkowski L., Korytkowski M., Scherer R., Tadeusiewicz R., Zadeh L.A., Zurada J.M. (eds) Artificial Intelligence and Soft Computing. ICAISC 2013. Lecture Notes in Computer Science, vol. 7894. Springer, Berlin, Heidelberg, pp. 52-63, 2013.

9. A. Dudek, H. Hurd and W. Wojtowicz, PARMA methods based on Fourier representation of periodic coefficients, Wiley Interdisciplinary Reviews: Computational Statistics, vol. 8, no. 3, pp. 130-149, 2016.

10. J. Franke, Minimax-robust prediction of discrete time series, Z. Wahrscheinlichkeitstheor. Verw. Gebiete, vol. 68, no. 3, pp. 337-364, 1985.

11. I. I. Gikhman and A. V. Skorokhod, The theory of stochastic processes. I., Berlin: Springer, 574 p., 2004.

12. L. Giraitis and R. Leipus, A generalized fractionally differencing approach in long-memory modeling, Lithuanian Mathematical Journal, vol. 35, pp. 53-65, 1995.

13. E. G. Gladyshev, Periodically correlated random sequences, Sov. Math. Dokl. vol, 2, pp. 385-388, 1961.

14. P. G. Gould, A. B. Koehler, J. K. Ord, R. D. Snyder, R. J. Hyndman and F. Vahid-Araghi, Forecasting time-series with multiple seasonal patterns, European Journal of Operational Research, vol. 191, pp. 207-222, 2008.

15. C. W. J. Granger and R. Joyeux, An intoduction to long memory time series and fractional differencing, Journal of Time Series Analysis, vol. 1, pp. 15-30, 1980.

16. U. Grenander, A prediction problem in game theory, Arkiv för Matematik, vol. 3, pp. 371-379, 1957

17. H. Gray, Q. Cheng and W. Woodward, On generalized fractional processes, Journal of Time Series Analysis, vol. 10, no. 3, pp. 233-257, 1989.

18. E. J. Hannan, Multiple time series. 2nd rev. ed., John Wiley \& Sons, New York, 536 p., 2009.

19. U. Hassler, Time series analysis with Long Memory in view, Wiley, Hoboken, NJ, 288 p., 2019.

20. U. Hassler and M.O. Pohle, Forecasting under long memory and nonstationarity, arXiv:1910.08202, 2019.

21. J. R. M. Hosking, Fractional differencing, Biometrika, vol. 68, no. 1, pp. 165-176, 1981.

22. Y. Hosoya, Robust linear extrapolations of second-order stationary processes, Annals of Probability, vol. 6, no. 4, pp. 574-584, 1978.

23. H. Hurd and V. Pipiras, Modeling periodic autoregressive time series with multiple periodic effects, In: Chaari F., Leskow J., Zimroz R., Wylomanska A., Dudek A. (eds) Cyclostationarity: Theory and Methods ?IV. CSTA 2017. Applied Condition Monitoring, vol 16. Springer, Cham, pp. 1-18, 2020.

24. S. Johansen and M. O. Nielsen, The role of initial values in conditional sum-of-squaresestimation of nonstationary fractional time series models, Econometric Theory, vol. 32, no. 5, pp. 1095-1139, 2016.

25. S. A. Kassam, Robust hypothesis testing and robust time series interpolation and regression, Journal of Time Series Analysis, vol. 3 , no. 3, pp. 185-194, 1982.

26. S.A. Kassam, and H. V. Poor, Robust techniques for signal processing: A survey, Proceedings of the IEEE, vol. 73, no. 3, pp. 433-481, 1985.

27. K. Karhunen, Uber lineare Methoden in der Wahrscheinlichkeitsrechnung, Annales Academiae Scientiarum Fennicae. Ser. A I, no. 37, 1947.

28. A. N. Kolmogorov, Selected works by A. N. Kolmogorov. Vol. II: Probability theory and mathematical statistics. Ed. by A. N. Shiryayev. Mathematics and Its Applications. Soviet Series. 26. Dordrecht etc. Kluwer Academic Publishers, 1992.

29. P. S. Kozak and M. P. Moklyachuk, Estimates of functionals constructed from random sequences with periodically stationary increments, Theory Probability and Mathematical Statistics, vol. 97, pp. 85-98, 2018.

30. R. Lund, Choosing seasonal autocovariance structures: PARMA or SARMA, In: Bell WR, Holan SH, McElroy TS (eds) Economic time series: modelling and seasonality. Chapman and Hall, London, pp. 63-80, 2011.

31. M. Luz and M. Moklyachuk, Minimax-robust prediction problem for stochastic sequences with stationary increments and cointegrated sequences, Statistics, Optimization and Information Compututing, vol. 3, no. 2, pp. 160-188, 2015.

32. M. Luz and M. Moklyachuk, Estimation of Stochastic Processes with Stationary Increments and Cointegrated Sequences, London: ISTE; Hoboken, NJ: John Wiley \& Sons, 282 p., 2019.

33. M. P. Moklyachuk, Minimax extrapolation and autoregressive-moving average processes, Theory of Probability and Mathematical Statistics, vol. 41, pp. 77-84, 1990.

34. M. P. Moklyachuk, Minimax-robust estimation problems for stationary stochastic sequences, Statistics, Optimization and Information Computing, vol. 3, no. 4, pp. 348-419, 2015.

35. M.P. Moklyachuk and A. Yu. Masyutka, Extrapolation of multidimensional stationary processes Random Operators and Stochastic Equations, vol. 14, no. 3, pp. 233-244, 2006.

36. M.P. Moklyachuk and A.Yu. Masyutka, Minimax prediction problem for multidimensional stationary stochastic processes, Communications in Statistics-Theory and Methods, vol. 40, no. 19-20, pp. 3700-3710, 2011.

37. M. Moklyachuk and M. Sidei, Extrapolation problem for stationary sequences with missing observations, Statistics, Optimization \& Information Computing, vol. 5, no. 3, pp. 212-233, 2017. 
38. M. Moklyachuk, M. Sidei and O. Masyutka, Estimation of stochastic processes with missing observations, Mathematics Research Developments. New York, NY: Nova Science Publishers, 336 p., 2019.

39. A. Napolitano, Cyclostationarity: New trends and applications, Signal Processing, vol. 120, pp. 385-408, 2016.

40. D. Osborn, The implications of periodically varying coefficients for seasonal time-series processes, Journal of Econometrics, vol. 48, no. 3, pp. 373-384, 1991.

41. W. Palma and P. Bondon, On the eigenstructure of generalized fractional processes, Statistics \& Probability Letters, vol. 65, pp. 93-101, 2003

42. M. S. Pinsker and A. M. Yaglom, On linear extrapolaion of random processes with nth stationary incremens, Doklady Akademii Nauk SSSR, n. Ser. vol. 94, pp. 385-388, 1954.

43. S. Porter-Hudak, An application of the seasonal fractionally differenced model to the monetary aggegrates, Journal of the American Statistical Association, vol.85, no. 410, pp. 338-344, 1990.

44. V. A. Reisen, B. Zamprogno, W. Palma and J. Arteche, A semiparametric approach to estimate two seasonal fractional parameters in the SARFIMA model, Mathematics and Computers in Simulation, vol. 98, pp. 1-17, 2014.

45. V. A. Reisen, E. Z. Monte, G. C. Franco, A. M. Sgrancio, F. A. F. Molinares, P. Bondon, F. A. Ziegelmann and B. Abraham, Robust estimation of fractional seasonal processes: Modeling and forecasting daily average SO2 concentrations, Mathematics and Computers in Simulation, vol. 146, pp. 27-43, 2018.

46. R. T. Rockafellar, Convex Analysis, Princeton Landmarks in Mathematics. Princeton, NJ: Princeton University Press, 451 p., 1997.

47. C. C. Solci, V. A. Reisen, A. J. Q. Sarnaglia and P. Bondon, Empirical study of robust estimation methods for PAR models with application to the air quality area, Communication in Statistics - Theory and Methods, vol. 48, no. 1, pp. 152-168, 2020.

48. H. Tsai, H. Rachinger and E.M.H. Lin, Inference of seasonal long-memory time series with measurement error, Scandinavian Journal of Statistics, vol. 42, no. 1, pp. 137-154, 2015.

49. S. K. Vastola and H. V. Poor, Robust Wiener-Kolmogorov theory, IEEE Trans. Inform. Theory, vol. 30, no. 2, pp. 316-327, 1984.

50. W. Woodward, Q. Cheng and H. Gray, A k-factor GARMA long memory model, Journal of Time Series Analysis, vol. 19, no. 4, pp. 485-504, 1998.

51. A. M. Yaglom, Correlation theory of stationary and related random processes with stationary nth increments, Mat. Sbornik, vol. 37, no. 1, pp. 141-196, 1955.

52. A. M. Yaglom, Correlation theory of stationary and related random functions. Vol. 1: Basic results; Vol. 2: Suplementary notes and references, Springer Series in Statistics, Springer-Verlag, New York etc., 1987. 